Supporting Information

\title{
Electrocatalytic C-N Coupling via Anodically Generated Hypervalent Iodine Intermediates
}

\author{
Asim Maity, Brandon L. Frey, Nathanael D. Hoskinson, and David C. Powers* \\ Department of Chemistry, Texas A\&M University, College Station, Texas 77843, United \\ States
}

Email: powers@chem.tamu.edu 
Table of Contents

A. General Considerations

A.1 Materials

A.2 Characterization Details

B. Synthesis and Characterization

B.1 Synthesis of Starting Materials

B.2 Intramolecular C-H Amination Electrocatalysis 13

B.3 Intermolecular C-H Amination Electrocatalysis 17

B.3.1 Derivatization of Intermolecular C-H Amination Products 18

C. Supporting Data

20

D. Additional Data

50

E. NMR Spectra of New Compounds

58

F. References

82 


\section{A. General Considerations}

Materials All chemicals and solvents were obtained as ACS reagent grade and used as received. 2-Bromoaniline, 4-tert-butylphenylboronic acid, 2,4-dimethylphenylboronic acid, 3-bromo-5-methylanisole, tetrabutylammonium hexafluorophosphate ([TBA] $\mathrm{PF}_{6}$ ), tetrabutylammonium acetate ([TBA]OAc), pyridine, and benzoyl chloride $(\mathrm{BzCl})$ were obtained from Tokyo Chemical Industry (TCI). 2-Bromo-4-fluoroaniline, 2-bromo- $p$ anisidine, 2-bromo-4-nitroaniline, 4-fluorophenylboronic acid, 4-chlorophenylboronic acid, 4-trifluoromethylphenylboronic acid, 2-chlorophenylboronic acid, trifluoroacetic acid, 4trifluoromethyliodobenzene, and 1,1,1,3,3,3-hexafluoro-2-phenyl-2-propanol were obtained from Matrix Scientific. 1,1,1,3,3,3-Hexafluoro-2-isopropanol (hfip) was acquired from Oakwood Chemicals. Palladium acetate $\left(\mathrm{Pd}(\mathrm{OAc})_{2}\right)$ was acquired from Strem Chemicals. 2-Bromo- $p$-toluidine, methyl-4-amino-3-bromobenzoate, 4-biphenylboronic acid, 1,2-diiodobenzene, and trimethyl borate were obtained from Alfa Aesar. 2-Bromo-4chloroaniline, 1,2-diiodobenzene, boron trifluoride diethyl etherate, [1,1'-biphenyl]-2-amine (S1a), and silica gel $(0.060-0.200 \mathrm{~mm}, 60 \mathrm{~A}$ for column chromatography) were obtained from Acros Organics. Tetrabutylammonium bromide ([TBA]Br), 4-methoxyphenylboronic acid, methanesulfonyl chloride (MsCl), and 2,3-dichloro-5,6-dicyano-1,4-benzoquinone (DDQ) were obtained from Chem-Impex Int'l Inc. Iodobenzene, 4-iodoanisole, 4-iodotoluene, 4-fluoroiodobenzene, 2,6-dimethoxyiodobenzene, ethyl 3-iodobenzoate, 4bromophenylboronic acid, 2,6-dibromoaniline, 2-iodobenzoic acid, $\mathrm{Pd}\left(\mathrm{PPh}_{3}\right)_{2} \mathrm{Cl}_{2}$, Raney $\mathrm{Ni}$, hydrazine monohydrate, tosyl chloride (TsCl), tetrahydrofuran (THF), hexanes, ethyl acetate, dichloromethane, $N, N$-dimethylformamide (DMF), acetic acid, acetic anhydride, and trifluoroacetic anhydride were obtained from Sigma Aldrich. Hydrochloric acid (36.5\%), $\mathrm{N}$ bromosuccinimide (NBS), anhydrous magnesium sulfate, anhydrous sodium carbonate, and anhydrous potassium carbonate were obtained from VWR. Sodium hydroxide was obtained from EMD Millipore. Acetonitrile and ethanol (200 proof) was obtained from Fischer Scientific. NMR solvents were purchased from Cambridge Isotope Laboratories and were used as received. All reactions were carried out under ambient atmosphere unless otherwise noted. 2-(2-Iodophenyl)propan-2-ol, ${ }^{1} \quad 2,2^{\prime}$-diiodo-4,4',6,6'-tetramethyl-1,1'-biphenyl, ${ }^{2}$ 1,1,1,3,3,3-hexafluoro-2-(2-iodophenyl)propan-2-ol, ${ }^{3} \quad N$-(1,3-dioxoisoindolin-2-yl)- $N$ phenylacetamide, $^{4} \quad$ 4-amino-3-bromobenzaldehyde, ${ }^{5}$ 2,4-dimethoxyiodobenzene, ${ }^{6}$ and $\operatorname{Pd}\left(\mathrm{PPh}_{3}\right)_{4}{ }^{7}$ were prepared according to literature procedures.

Characterization Details ${ }^{1} \mathrm{H}$ and ${ }^{13} \mathrm{C}$ NMR spectral acquisitions were recorded on an Inova 500 FT NMR (Varian), a VNMRS 500 FT NMR (Varian), or an Acsend ${ }^{\mathrm{TM}} 400$ NMR (Bruker) and were referenced against residual proteo solvent signals: $\mathrm{CDCl}_{3}\left(7.26 \mathrm{ppm},{ }^{1} \mathrm{H} ; 77.16\right.$ $\left.\mathrm{ppm},{ }^{13} \mathrm{C}\right)$ and acetonitrile- $d_{3}\left(1.94 \mathrm{ppm},{ }^{1} \mathrm{H}\right) .{ }^{8}{ }^{1} \mathrm{H}$ NMR data are reported as follows: chemical shift ( $\delta, \mathrm{ppm}),($ multiplicity: $\mathrm{s}$ (singlet), d (doublet), $\mathrm{t}$ (triplet), $\mathrm{m}$ (multiplet), br (broad), integration). ${ }^{13} \mathrm{C}$ NMR data are reported as follows: chemical shift $(\delta, \mathrm{ppm})$. Mass spectrometry data was recorded on either Orbitrap Fusion ${ }^{\mathrm{TM}}$ Tribrid $^{\mathrm{TM}}$ Mass Spectrometer or Q Exactive ${ }^{\mathrm{TM}}$ Focus Hybrid Quadrupole-Orbitrap ${ }^{\mathrm{TM}}$ Mass Spectrometer from ThermoFisher Scientific. An Agilent Trace 1300 GC with attached thermal conductivity detector and a custom-made $120 \mathrm{~cm}$ stainless steel column packed with Carbosieve-II was used for analysis of headspace gases. The column was kept at $200{ }^{\circ} \mathrm{C}$ and $\mathrm{Ar}$ was used as 
carrier gas. The detector was set to a temperature of $250{ }^{\circ} \mathrm{C}$. Headspace gas $(\sim 300 \mu \mathrm{L})$ was transferred to the GC with a $0.50 \mathrm{~mL}$ Valco Precision Sampling Syringe (Series A-2) equipped with a Valco Precision sampling needle with a 5-point side port. Cyclic voltammetry (CV) experiments were carried out using $\mathrm{CH}$ Instruments Electrochemical Analyzer (Model CHI620A) in a three-electrode cell. For CV experiments, a glassy carbon working electrode, Pt counter electrode, and Ag-reference electrode were used (obtained from CH Instruments). For both constant potential and constant current electrolysis, glassy carbon, Pt, boron-doped carbon, and graphite working electrodes were purchased from IKA. An IKA Electrasyn 2.0 was used for constant current electrolysis and $\mathrm{CH}$ Instruments Electrochemical Analyzer (Model CHI620A) potentiostat was used for constant potential electrolysis. All electrochemical experiments were carried out in 10-mL Electrasyn glass vial. Reference electrodes were prepared using $0.1 \mathrm{M}$ solution of [TBA] $\mathrm{PF}_{6}$ in acetonitrile with $1.0 \mathrm{mM}$ $\mathrm{AgNO}_{3}$. 


\section{B. Synthesis and Characterization}

\section{B.1 Synthesis of Starting Materials}

\section{General Procedure for the Synthesis of Biarylanilines (S1)}

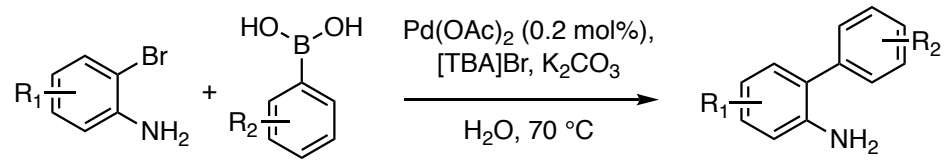

A 20 -mL scintillation vial was charged with the appropriate substituted 2-bromoaniline ( $2.80 \mathrm{mmol}, 1.00$ equiv.), the appropriate substituted phenylboronic acid $(4.20 \mathrm{mmol}, 1.50$ equiv.), $\mathrm{Pd}(\mathrm{OAc})_{2}(1.3 \mathrm{mg}, 6.0 \mu \mathrm{mol}, 0.20 \mathrm{~mol} \%$ ), [TBA]Br ( $903 \mathrm{mg}, 2.90 \mathrm{mmol}, 1.00$ equiv.), $\mathrm{K}_{2} \mathrm{CO}_{3}$ (977 mg, $7.07 \mathrm{mmol}, 2.52$ equiv.), and distilled water $(4 \mathrm{~mL})$. The reaction vessel was fitted with a rubber septum, purged with $\mathrm{N}_{2}$ for $10 \mathrm{~min}$, and the reaction mixture was heated at $70{ }^{\circ} \mathrm{C}$ for $16 \mathrm{~h}$. The reaction mixture was cooled to $23{ }^{\circ} \mathrm{C}$ and was extracted with ethyl acetate $(3 \times 10 \mathrm{~mL})$. The combined organic fractions were dried over $\mathrm{MgSO}_{4}$ and solvent was removed under reduced pressure. The obtained residue was purified by $\mathrm{SiO}_{2}$ gel chromatography (eluent 95:5 hexanes:ethyl acetate) to afford the biarylamines listed below.

5-Chloro-[1,1'-biphenyl]-2-amine (S1c). Prepared from 2-bromo-4-chloroaniline and phenylboronic acid, S1c was obtained as a colorless oil (75\% yield). ${ }^{1} \mathrm{H}$ NMR $\left(\delta, 23{ }^{\circ} \mathrm{C}, 400\right.$ $\left.\mathrm{MHz}, \mathrm{CDCl}_{3}\right)$ : 7.51-7.43 (m, 4H), 7.42-7.38 (m, 1H), 7.16-7.09 (m, 2H), 6.73-6.69 (m, 1H), $3.76(\mathrm{br}, 2 \mathrm{H})$. The obtained spectral data are in good agreement with those reported in literature. ${ }^{9}$

5-Fluoro-[1,1'-biphenyl]-2-amine (S1d). Prepared from 2-bromo-4-fluoroaniline and phenylboronic acid, $\mathbf{S 1 d}$ was obtained as a colorless oil (69\% yield). ${ }^{1} \mathrm{H}$ NMR $\left(\delta, 23{ }^{\circ} \mathrm{C}, 400\right.$ $\left.\mathrm{MHz}, \mathrm{CDCl}_{3}\right)$ : 7.51-7.46 (m, 4H), 7.42-7.38 (m, 1H), 6.92-6.88 (m, 2H), 6.73-6.69 (m, 1H), $3.64(\mathrm{br}, 2 \mathrm{H})$. The obtained spectral data are in good agreement with those reported in literature. ${ }^{10}$

4'-Chloro-[1,1'-biphenyl]-2-amine (S1f). Prepared from 2-bromoaniline and 4chlorophenylboronic acid, S1f was obtained as a colorless oil (41\% yield). ${ }^{1} \mathrm{H}$ NMR $\left(\delta, 23^{\circ} \mathrm{C}\right.$, $400 \mathrm{MHz}, \mathrm{CDCl}_{3}$ ): 7.44-7.39 (m, 4H), 7.20-7.15 (m, 1H), 7.10 (dd, J = 7.6, $\left.1.3 \mathrm{~Hz}, 1 \mathrm{H}\right), 6.83(\mathrm{td}$, $J=7.5,1.1 \mathrm{~Hz}, 1 \mathrm{H}), 6.77(\mathrm{dd}, J=8.0,0.9 \mathrm{~Hz}, 1 \mathrm{H}), 3.72(\mathrm{br}, 2 \mathrm{H})$. The obtained spectral data are in good agreement with those reported in literature. ${ }^{5}$

4'-Fluoro-[1,1'-biphenyl]-2-amine (S1g). Prepared from 2-bromoaniline and 4fluorophenylboronic acid, S1g was obtained as a colorless oil (94\% yield). ${ }^{1} \mathrm{H}$ NMR $\left(\delta, 23^{\circ} \mathrm{C}\right.$, $400 \mathrm{MHz}, \mathrm{CDCl}_{3}$ ): 7.44 (dd, $\left.J=8.8,5.5 \mathrm{~Hz}, 2 \mathrm{H}\right), 7.18-7.10(\mathrm{~m}, 4 \mathrm{H}), 6.84(\mathrm{td}, J=7.5,1.2 \mathrm{~Hz}, 1 \mathrm{H})$, $6.78(\mathrm{dd}, J=8.0,1.1 \mathrm{~Hz}, 1 \mathrm{H}), 3.72(\mathrm{br}, 2 \mathrm{H})$. The obtained spectral data are in good agreement with those reported in literature. ${ }^{5}$

6-Amino-[1,1'-biphenyl]-3-carbaldehyde (S1h). Prepared from 4-amino-3bromobenzaldehyde and phenylboronic acid, S1h was obtained as a colorless oil $(71 \%$ yield). ${ }^{1} \mathrm{H} \mathrm{NMR}\left(\delta, 23{ }^{\circ} \mathrm{C}, 400 \mathrm{MHz}, \mathrm{CDCl}_{3}\right): 9.80(\mathrm{~s}, 1 \mathrm{H}), 7.71(\mathrm{dd}, J=8.3,1.9 \mathrm{~Hz}, 1 \mathrm{H}), 7.66(\mathrm{~d}$, $J=1.8 \mathrm{~Hz}, 1 \mathrm{H}), 7.51-7.41(\mathrm{~m}, 5 \mathrm{H}), 6.81(\mathrm{~d}, J=8.3 \mathrm{~Hz}, 1 \mathrm{H}), 4.44(\mathrm{br}, 2 \mathrm{H}) .{ }^{13} \mathrm{C}$ NMR $\left(\delta, 23^{\circ} \mathrm{C}\right.$, 
$100 \mathrm{MHz}, \mathrm{CDCl}_{3}$ ): 190.5, 149.6, 137.9, 133.25, 133.24, 130.9, 129.2, 128.9, 127.91, 127.77, 126.8, 114.7. HRMS-ESI: calculated for $[\mathrm{M}+\mathrm{H}]=196.0910$, observed $[\mathrm{M}+\mathrm{H}]=196.0913$.

[1,1':4',1'-Terphenyl]-2-amine (S1i). Prepared from 2-bromoaniline and 4-biphenylboronic acid, S1i was obtained as a colorless oil $\left(64 \%\right.$ yield). ${ }^{1} \mathrm{H}$ NMR $\left(\delta, 23{ }^{\circ} \mathrm{C}, 400 \mathrm{MHz}, \mathrm{CDCl}_{3}\right)$ : 7.71-7.66 (m, 4H), 7.58-7.55 (m, 2H), 7.49 (t, J= 7.6 Hz, 2H), 7.41-7.37 (m, 1H), 7.22-7.18 $(\mathrm{m}, 2 \mathrm{H}), 6.89-6.86(\mathrm{~m}, 1 \mathrm{H}), 6.83-6.81(\mathrm{~m}, 1 \mathrm{H}), 3.83(\mathrm{br}, 1 \mathrm{H})$. The obtained spectral data are in good agreement with those reported in literature. ${ }^{9}$

5-Nitro-[1,1'-biphenyl]-2-amine (S1j). Prepared from 2-bromo-4-nitroaniline and phenylboronic acid, $\mathbf{S 1 j}$ was obtained as a yellow oil $(71 \%$ yield $) .{ }^{1} \mathrm{H} N \mathrm{NR}\left(\delta, 23{ }^{\circ} \mathrm{C}, 400 \mathrm{MHz}\right.$, $\left.\mathrm{CDCl}_{3}\right)$ : 8.09-8.06 (m, 2H), 7.52-7.48 (m, 2H), 7.44-7.40 (m, 3H), 6.73-6.71 (m, 1H), $4.48(\mathrm{br}$, $2 \mathrm{H})$. The obtained spectral data are in good agreement with those reported in literature. ${ }^{11}$

Methyl 6-amino-[1,1'-biphenyl]-3-carboxylate (S1k). Prepared from methyl 4-amino-3bromobenzoate and phenylboronic acid, S1k was obtained as a colorless oil (61\% yield). ${ }^{1} \mathrm{H}$ $\operatorname{NMR}\left(\delta, 23{ }^{\circ} \mathrm{C}, 400 \mathrm{MHz}, \mathrm{CDCl}_{3}\right)$ : 7.85-7.83 (m, 2H), 7.47-7.45 (m, 4H), 7.40-7.35 (m, $\left.2 \mathrm{H}\right)$, $6.75(\mathrm{~d}, J=9.0 \mathrm{~Hz}, 1 \mathrm{H}), 4.26(\mathrm{br}, 2 \mathrm{H}), 3.86(\mathrm{~s}, 3 \mathrm{H})$.The obtained spectral data are in good agreement with those reported in literature. ${ }^{12}$

4'-(Tert-butyl)-[1,1'-biphenyl]-2-amine (S11). Prepared from 2-bromoaniline and 4-tertbutylphenylboronic acid, S1l was obtained as a colorless oil (59\% yield). ${ }^{1} \mathrm{H}$ NMR $\left(\delta, 23^{\circ} \mathrm{C}\right.$, $\left.400 \mathrm{MHz}, \mathrm{CDCl}_{3}\right): 7.47(\mathrm{~d}, J=8.6 \mathrm{~Hz}, 2 \mathrm{H}), 7.40(\mathrm{~d}, J=8.6 \mathrm{~Hz}, 2 \mathrm{H}), 7.15(\mathrm{td}, J=7.2,1.6 \mathrm{~Hz}, 2 \mathrm{H})$, 6.84-6.76 (m, 2H), $3.78(\mathrm{br}, 2 \mathrm{H}), 1.37(\mathrm{~s}, 9 \mathrm{H})$. The obtained spectral data are in good agreement with those reported in literature. ${ }^{9}$

4'-Methoxy-[1,1'-biphenyl]-2-amine (S1m). Prepared from 2-bromoaniline and 4methoxyphenylboronic acid, S1m was obtained as a colorless oil (76\% yield). ${ }^{1} \mathrm{H}$ NMR $(\delta, 23$ $\left.{ }^{\circ} \mathrm{C}, 400 \mathrm{MHz}, \mathrm{CDCl}_{3}\right): 7.43-7.41(\mathrm{~m}, 2 \mathrm{H}), 7.19-7.14(\mathrm{~m}, 2 \mathrm{H}), 7.03-7.01(\mathrm{~m}, 2 \mathrm{H}), 6.85(\mathrm{td}, J=$ $7.4,1.2 \mathrm{~Hz}, 1 \mathrm{H}), 6.79-6.77(\mathrm{~m}, 1 \mathrm{H}), 3.88(\mathrm{~s}, 3 \mathrm{H}), 3.77(\mathrm{br}, 2 \mathrm{H})$. The obtained spectral data are in good agreement with those reported in literature. ${ }^{9}$

5-Methoxyl-[1,1'-biphenyl]-2-amine (S1n). Prepared from 2-bromo-4-methoxyaniline and phenylboronic acid, S1n was obtained as a yellow oil (53\% yield). ${ }^{1} \mathrm{H}$ NMR $\left(\delta, 23{ }^{\circ} \mathrm{C}, 400\right.$ $\left.\mathrm{MHz}, \mathrm{CDCl}_{3}\right): 7.50-7.45(\mathrm{~m}, 4 \mathrm{H}), 7.40-7.36(\mathrm{~m}, 1 \mathrm{H}), 6.82-6.73(\mathrm{~m}, 3 \mathrm{H}), 3.79(\mathrm{~s}, 3 \mathrm{H}), 3.53(\mathrm{br}$, $2 \mathrm{H})$. The obtained spectral data are in good agreement with those reported in literature. ${ }^{13}$

5-Methyl-[1,1'-biphenyl]-2-amine (S1o). Prepared from 2-bromo-4-methylaniline and phenylboronic acid, S1o was obtained as a light orange oil (53\% yield). ${ }^{1} \mathrm{H}$ NMR $\left(\delta, 23{ }^{\circ} \mathrm{C}\right.$, $\left.400 \mathrm{MHz}, \mathrm{CDCl}_{3}\right):$ 7.53-7.47 (m, 4H), 7.41-7.37 (m, 1H), 7.04-7.02 (m, 2H), $6.74(\mathrm{~d}, J=8.3$ $\mathrm{Hz}, 1 \mathrm{H}), 3.58(\mathrm{br}, 2 \mathrm{H}), 2.34(\mathrm{~s}, 3 \mathrm{H})$. The obtained spectral data are in good agreement with those reported in literature. ${ }^{10}$

5-(Tert-butyl)-[1,1'-biphenyl]-2-amine (S1p). Prepared from 2-bromo-4-(tert-butyl)aniline and phenylboronic acid, S1p was obtained as a colorless solid (70\% yield). ${ }^{1} \mathrm{H}$ NMR $\left(\delta, 23^{\circ} \mathrm{C}\right.$, $400 \mathrm{MHz}, \mathrm{CDCl}_{3}$ ): 7.53-7.46 (m, 4H), 7.38 (ddt, $J=7.7,6.2,1.6 \mathrm{~Hz}, 1 \mathrm{H}$ ), 7.23 (dd, $J=8.3,2.4$ $\mathrm{Hz}, 1 \mathrm{H}$ ), 7.19 (d, $J=2.3 \mathrm{~Hz}, 1 \mathrm{H}), 6.77(\mathrm{~d}, J=8.3 \mathrm{~Hz}, 1 \mathrm{H}$ ), 3.69 (br, 2H), $1.34(\mathrm{~s}, 9 \mathrm{H}$ ). The obtained spectral data are in good agreement with those reported in literature. ${ }^{11}$ 
2'-Chloro-[1,1'-biphenyl]-2-amine (S1q). Prepared from 2-bromoaniline and 2chlorophenylboronic acid, S1q was obtained as a colorless solid (36\% yield). ${ }^{1} \mathrm{H}$ NMR $(\delta, 23$ ${ }^{\circ} \mathrm{C}, 400 \mathrm{MHz}, \mathrm{CDCl}_{3}$ ): $7.51(\mathrm{dt}, J=6.9,1.6 \mathrm{~Hz}, 1 \mathrm{H}$ ), 7.36-7.30 (m, 3H), 7.22 (ddd, $J=8.0,7.4$, $1.6 \mathrm{~Hz}, 1 \mathrm{H}), 7.07-7.05(\mathrm{~m}, 1 \mathrm{H}), 6.86-6.79(\mathrm{~m}, 2 \mathrm{H}), 3.27(\mathrm{~s}, 2 \mathrm{H})$. The obtained spectral data are in good agreement with those reported in literature. ${ }^{14}$

4,4"-Dichloro-[1,1':3',1"'-terphenyl]-2'-amine (S1r). Prepared from 2,6-dibromoaniline and 4chlorophenylboronic acid, S1r was obtained as a light yellow oil (51\% yield). ${ }^{1} \mathrm{H}$ NMR $(\delta, 23$ $\left.{ }^{\circ} \mathrm{C}, 400 \mathrm{MHz}, \mathrm{CDCl}_{3}\right): 7.46(\mathrm{dd}, J=8.8,5.4 \mathrm{~Hz}, 4 \mathrm{H}), 7.15(\mathrm{t}, J=8.8 \mathrm{~Hz}, 4 \mathrm{H}), 7.09(\mathrm{~d}, J=7.5 \mathrm{~Hz}$, $2 \mathrm{H}), 6.87(\mathrm{dd}, J=7.8,7.3 \mathrm{~Hz}, 1 \mathrm{H}), 3.49(\mathrm{~s}, 2 \mathrm{H})$. The obtained spectral data are in good agreement with those reported in literature. ${ }^{15}$

4,4"'-Difluoro-[1,1':3',1"'-terphenyl]-2'-amine (S1s). Prepared from 2,6-dibromoaniline and 4fluorophenylboronic acid, S1s was obtained as a colorless solid (45\% yield). ${ }^{1} \mathrm{H}$ NMR $(\delta, 23$ $\left.{ }^{\circ} \mathrm{C}, 400 \mathrm{MHz}, \mathrm{CDCl}_{3}\right): 7.43$ (s, 8H), 7.09 (d, $\left.J=7.5 \mathrm{~Hz}, 2 \mathrm{H}\right), 6.87(\mathrm{t}, J=7.5 \mathrm{~Hz}, 1 \mathrm{H}), 3.76(\mathrm{~s}, 2 \mathrm{H})$. The obtained spectral data are in good agreement with those reported in literature. ${ }^{15}$

4',5-Dichloro-[1,1'-biphenyl]-2-amine (S1t). Prepared from 2-bromo-4-chloroaniline and 4chlorophenylboronic acid, S1t was obtained as a colorless solid (40\% yield). ${ }^{1} \mathrm{H}$ NMR $(\delta, 23$ $\left.{ }^{\circ} \mathrm{C}, 400 \mathrm{MHz}, \mathrm{CDCl}_{3}\right)$ : 7.44-7.41 (m, 2H), 7.41-7.35 (m, 2H), 7.11 (dd, $\left.J=8.5,2.5 \mathrm{~Hz}, 1 \mathrm{H}\right), 7.10-$ $7.06(\mathrm{~m}, 1 \mathrm{H}), 6.68(\mathrm{~d}, J=8.5 \mathrm{~Hz}, 1 \mathrm{H}), 3.70(\mathrm{br}, 2 \mathrm{H})$. The obtained spectral data are in good agreement with those reported in literature. ${ }^{16}$

4'-Chloro-5-fluoro-[1,1'-biphenyl]-2-amine (S1u). Prepared from 2-bromo-4-fluoroaniline and 4-chlorophenylboronic acid, $\mathbf{S 1} \mathbf{u}$ was obtained as a colorless solid (63\% yield). ${ }^{1} \mathrm{H}$ NMR $\left(\delta, 23{ }^{\circ} \mathrm{C}, 400 \mathrm{MHz}, \mathrm{CDCl}_{3}\right): 7.45-7.41(\mathrm{~m}, 2 \mathrm{H}), 7.41-7.37(\mathrm{~m}, 2 \mathrm{H}), 6.88(\mathrm{td}, J=8.4,3.0 \mathrm{~Hz}, 1 \mathrm{H})$, $6.83(\mathrm{dd}, J=9.2,2.9 \mathrm{~Hz}, 1 \mathrm{H}), 6.69(\mathrm{dd}, J=8.7,4.8 \mathrm{~Hz}, 1 \mathrm{H}), 3.58(\mathrm{br}, 2 \mathrm{H})$. The obtained spectral data are in good agreement with those reported in literature. ${ }^{16}$ 


\section{Synthesis of 5-bromo-[1,1'-biphenyl]-2-amine (S1b)}

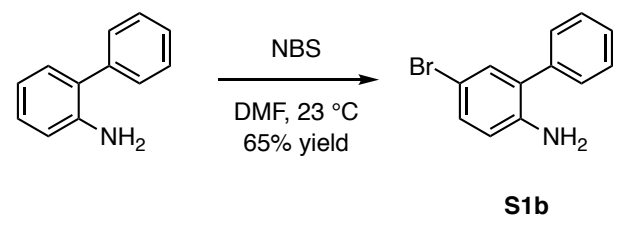

Compound S1b was synthesized according to the following modification of a literature procedure. ${ }^{17} \mathrm{~A} 50$ - $\mathrm{mL}$ round-bottom flask was charged with [1,1'-biphenyl]-2-amine (850 $\mathrm{mg}, 5.00 \mathrm{mmol}, 1.00$ equiv.) and DMF $(20 \mathrm{~mL})$. The resulting solution was cooled to $0{ }^{\circ} \mathrm{C}$ with an ice bath. A solution of NBS (1.05 g, $6.00 \mathrm{mmol}, 1.20$ equiv.) dissolved in $10 \mathrm{~mL}$ of DMF was added to the reaction vessel dropwise at $0{ }^{\circ} \mathrm{C}$. The reaction mixture was allowed to warm to $23^{\circ} \mathrm{C}$ and was stirred for $2 \mathrm{~h}$. The reaction mixture was diluted with distilled water $(\sim 50$ $\mathrm{mL})$ and extracted with $\mathrm{CH}_{2} \mathrm{Cl}_{2}(3 \times 10 \mathrm{~mL})$. The combined organic fractions were dried over $\mathrm{MgSO}_{4}$ and solvent was removed under reduced pressure. The obtained residue was purified by $\mathrm{SiO}_{2}$ gel chromatography (eluent 95:5 hexanes:ethyl acetate) to afford the desired product as yellow solid ( $810 \mathrm{mg}, 65 \%$ yield). ${ }^{1} \mathrm{H} \mathrm{NMR}\left(\delta, 23{ }^{\circ} \mathrm{C}, 400 \mathrm{MHz}, \mathrm{CDCl}_{3}\right): 7.47-7.34$ $(\mathrm{m}, 6 \mathrm{H}), 7.26-7.23(\mathrm{~m}, 1 \mathrm{H}), 6.67(\mathrm{~d}, J=9.1 \mathrm{~Hz}, 1 \mathrm{H}), 3.94(\mathrm{br}, 2 \mathrm{H})$. The obtained spectral data are in good agreement with those reported in literature. ${ }^{18}$

\section{Synthesis of 4'-bromo-[1,1'-biphenyl]-2-amine (S1e)}

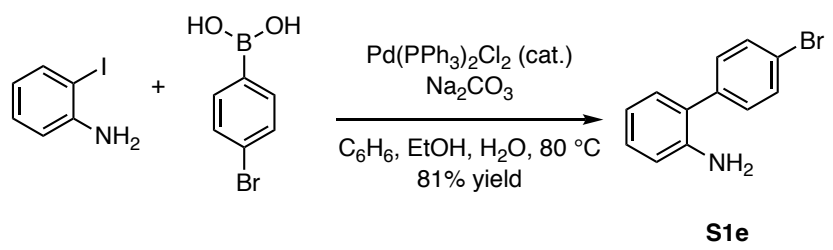

Compound S1e was synthesized according to the following modification of a literature procedure. ${ }^{19} \mathrm{~A} 100-\mathrm{mL}$ round-bottom flask was charged with 2-iodoaniline $(619 \mathrm{mg}, 2.83$ mmol, 1.00 equiv.), 4-bromophenylboronic acid (699 mg, $3.49 \mathrm{mmol}, 1.23$ equiv.), $\mathrm{Pd}\left(\mathrm{PPh}_{3}\right)_{2} \mathrm{Cl}_{2}$ (181 mg, $0.258 \mathrm{mmol}, 9.14 \mathrm{~mol} \%$ ), $\mathrm{Na}_{2} \mathrm{CO}_{3}$ (1.01 g, $9.61 \mathrm{mmol}, 3.4$ equiv.), benzene $(25 \mathrm{~mL})$, ethanol $(1.5 \mathrm{~mL})$, and distilled water $(5 \mathrm{~mL})$. The reaction vessel was fitted with a rubber septum, purged with $\mathrm{N}_{2}$ for $10 \mathrm{~min}$, and heated at $80^{\circ} \mathrm{C}$ for $16 \mathrm{~h}$. The reaction mixture was cooled to $23{ }^{\circ} \mathrm{C}$ and was then extracted with ethyl acetate $(3 \times 20 \mathrm{~mL})$. The combined organic fractions were dried over $\mathrm{MgSO}_{4}$ and solvent was removed under reduced pressure. The obtained residue was purified by $\mathrm{SiO}_{2}$ gel chromatography (eluent 95:5 hexanes:ethyl acetate) to afford the title compound as white solid (570 mg, $81 \%$ yield). ${ }^{1} \mathrm{H}$ $\operatorname{NMR}\left(\delta, 23{ }^{\circ} \mathrm{C}, 400 \mathrm{MHz}, \mathrm{CDCl}_{3}\right): 7.59(\mathrm{~d}, J=8.5 \mathrm{~Hz}, 2 \mathrm{H}), 7.37(\mathrm{~d}, J=8.5 \mathrm{~Hz}, 2 \mathrm{H}), 7.19$ (td, $J=$ 7.7, $1.4 \mathrm{~Hz}, 1 \mathrm{H}$ ), 7.11 (dd, $J=7.6,1.6 \mathrm{~Hz}, 1 \mathrm{H}), 6.86$ (dd, $J=7.5,1.2 \mathrm{~Hz}, 1 \mathrm{H}$ ), 6.79 (dd, $J=8.0$, $1.0 \mathrm{~Hz}, 1 \mathrm{H}), 3.74(\mathrm{~s}, 2 \mathrm{H})$. The obtained spectral data are in good agreement with those reported in literature. ${ }^{19}$ 


\section{General Procedure for Acylation of Biarylanilines}

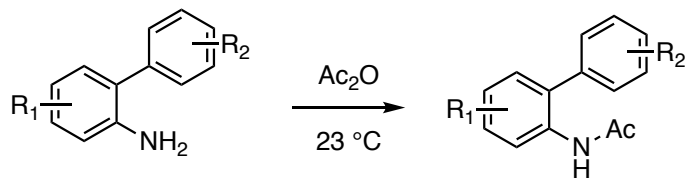

A $20-\mathrm{mL}$ scintillation vial was charged with the appropriate biarylamine $(2.0 \mathrm{mmol}, 1.0$ equiv.) and acetic anhydride $\left(\mathrm{Ac}_{2} \mathrm{O}, \sim 2 \mathrm{~mL}\right)$. The reaction mixture was stirred at $23{ }^{\circ} \mathrm{C}$ for 10 min. The reaction mixture was poured onto a pre-cooled saturated solution of $\mathrm{K}_{2} \mathrm{CO}_{3}\left(0^{\circ} \mathrm{C}\right)$. The resulting precipitate was isolated by vacuum filtration, washed with distilled water $(2 \times$ $5 \mathrm{~mL})$ and hexanes $(2 \times 5 \mathrm{~mL})$, and dried under reduced pressure to provide the $N$ arylacetamides listed below.

$\mathrm{N}$-([1,1'-biphenyl]-2-yl)acetamide (1a). Prepared from [1,1'-biphenyl]-2-amine (S1a), 1a was obtained as a white solid (82\% yield). ${ }^{1} \mathrm{H}$ NMR $\left(\delta, 23{ }^{\circ} \mathrm{C}, 400 \mathrm{MHz}, \mathrm{CDCl}_{3}\right): 8.27(\mathrm{~d}, J=8.0 \mathrm{~Hz}$, $1 \mathrm{H}), 7.49$ (t, J = 7.3 Hz, 2H), 7.44-7.37 (m, 4H), 7.24-7.18 (m, 2H), $7.11(\mathrm{br}, 1 \mathrm{H}), 2.02(\mathrm{~s}, 3 \mathrm{H})$. The obtained spectral data are in good agreement with those reported in literature. ${ }^{20}$

N-(5-bromo-[1,1'-biphenyl]-2-yl)acetamide (1b). Prepared from 5-bromo-[1,1'-biphenyl]-2amine (S1b), 1b was obtained as a colorless solid (78\% yield). ${ }^{1} \mathrm{H}$ NMR $\left(\delta, 23{ }^{\circ} \mathrm{C}, 400 \mathrm{MHz}\right.$, $\mathrm{CDCl}_{3}$ ): $8.22(\mathrm{~d}, J=8.6 \mathrm{~Hz}, 1 \mathrm{H}), 7.52-7.44(\mathrm{~m}, 4 \mathrm{H}), 7.38-7.34(\mathrm{~m}, 3 \mathrm{H}), 7.08(\mathrm{br}, 1 \mathrm{H}), 2.01(\mathrm{~s}$, $3 \mathrm{H})$. The obtained spectral data are in good agreement with those reported in literature. ${ }^{20}$

N-(5-chloro-[1,1'-biphenyl]-2-yl)acetamide (1c). Prepared from 5-chloro-[1,1'-biphenyl]-2amine (S1c), 1c was obtained as a colorless solid (98\% yield). ${ }^{1} \mathrm{H}$ NMR $\left(\delta, 23{ }^{\circ} \mathrm{C}, 400 \mathrm{MHz}\right.$, $\left.\mathrm{CDCl}_{3}\right): 8.26(\mathrm{~d}, J=8.8 \mathrm{~Hz}, 1 \mathrm{H}), 7.53-7.43(\mathrm{~m}, 3 \mathrm{H}), 7.37-7.32(\mathrm{~m}, 3 \mathrm{H}), 7.23(\mathrm{~d}, J=2.4 \mathrm{~Hz}, 1 \mathrm{H})$, $7.08(\mathrm{br}, 1 \mathrm{H}), 2.02(\mathrm{~s}, 3 \mathrm{H})$. The obtained spectral data are in good agreement with those reported in literature. ${ }^{20}$

N-(5-fluoro-[1,1'-biphenyl]-2-yl)acetamide (1d). Prepared from 5-fluoro-[1,1'-biphenyl]-2amine (S1d), $1 \mathrm{~d}$ was obtained as a colorless solid (88\% yield). ${ }^{1} \mathrm{H}$ NMR $\left(\delta, 23{ }^{\circ} \mathrm{C}, 400 \mathrm{MHz}\right.$, $\mathrm{CDCl}_{3}$ ): $8.07(\mathrm{~m}, 1 \mathrm{H}), 7.47-7.29(\mathrm{~m}, 5 \mathrm{H}), 7.11-7.00(\mathrm{~m}, 2 \mathrm{H}), 6.94(\mathrm{br}, 1 \mathrm{H}), 2.05(\mathrm{~s}, 3 \mathrm{H})$. The obtained spectral data are in good agreement with those reported in literature. ${ }^{20}$

N-(4'-bromo-[1,1'-biphenyl]-2-yl)acetamide (1e). Prepared from 4'-bromo-[1,1'-biphenyl]-2amine (S1e), 1e was obtained as a white solid (76 \% yield). ${ }^{1} \mathrm{H}$ NMR $\left(\delta, 23{ }^{\circ} \mathrm{C}, 400 \mathrm{MHz}\right.$, $\mathrm{CDCl}_{3}$ ): 8.24-8.21 (m, 1H), 7.66-7.63 (m, 2H), 7.43-7.37 (m, 1H), 7.28-7.23 (m, 4H), 7.00 (br, $1 \mathrm{H}), 2.07(\mathrm{~s}, 3 \mathrm{H})$. The obtained spectral data are in good agreement with those reported in literature. ${ }^{20}$

N-(4'-chloro-[1,1'-biphenyl]-2-yl)acetamide (1f). Prepared from 4'-chloro-[1,1'-biphenyl]-2amine (S1f), 1f was obtained as a colorless solid (77\% yield). ${ }^{1} \mathrm{H}$ NMR $\left(\delta, 23{ }^{\circ} \mathrm{C}, 400 \mathrm{MHz}\right.$, $\mathrm{CDCl}_{3}$ ): $8.20(\mathrm{~d}, J=7.9 \mathrm{~Hz}, 1 \mathrm{H}), 7.46(\mathrm{~d}, J=8.4 \mathrm{~Hz}, 2 \mathrm{H}), 7.38(\mathrm{ddd}, J=8.4,6.5,2.1 \mathrm{~Hz}, 1 \mathrm{H}), 7.31$ $(\mathrm{d}, J=8.4 \mathrm{~Hz}, 2 \mathrm{H}), 7.20(\mathrm{~d}, J=7.0 \mathrm{~Hz}, 2 \mathrm{H}), 6.98(\mathrm{br}, 1 \mathrm{H}), 2.04(\mathrm{~s}, 3 \mathrm{H})$. The obtained spectral data are in good agreement with those reported in literature. ${ }^{20}$

N-(4'-fluoro-[1,1'-biphenyl]-2-yl)acetamide (1g). Prepared from 4'-fluoro-[1,1'-biphenyl]-2amine (S1g), $1 \mathrm{~g}$ was obtained as a colorless solid (85\% yield). ${ }^{1} \mathrm{H}$ NMR $\left(\delta, 23{ }^{\circ} \mathrm{C}, 400 \mathrm{MHz}\right.$, 
$\left.\mathrm{CDCl}_{3}\right): 8.20(\mathrm{~d}, J=8.0 \mathrm{~Hz}, 1 \mathrm{H}), 7.38-7.32(\mathrm{~m}, 3 \mathrm{H}), 7.20-7.15(\mathrm{~m}, 4 \mathrm{H}), 7.01(\mathrm{br}, 1 \mathrm{H}), 2.03(\mathrm{~s}$, $3 \mathrm{H})$. The obtained spectral data are in good agreement with those reported in literature. ${ }^{20}$

N-(5-formyl-[1,1'-biphenyl]-2-yl)acetamide (1h). Prepared from 6-amino-[1,1'-biphenyl]-3carbaldehyde (S1h), $\mathbf{1 h}$ was obtained as an orange solid (61\% yield). ${ }^{1} \mathrm{H}$ NMR $\left(\delta, 23{ }^{\circ} \mathrm{C}, 400\right.$ $\mathrm{MHz}_{\mathrm{CDCl}}$ ): $9.94(\mathrm{~s}, 1 \mathrm{H}), 8.61(\mathrm{~d}, J=8.4 \mathrm{~Hz}, 1 \mathrm{H}), 7.87(\mathrm{dd}, J=8.4,2.0 \mathrm{~Hz}, 1 \mathrm{H}), 7.76(\mathrm{~d}, J=2.0$ $\mathrm{Hz}, 1 \mathrm{H}$ ), 7.53 (dd, $J=8.0,6.5 \mathrm{~Hz}, 2 \mathrm{H}), 7.49-7.47$ (m, 1H), 7.42 (br, 1H), 7.39-7.37 (m, 3H), $2.05(\mathrm{~s}, 3 \mathrm{H})$. The obtained spectral data are in good agreement with those reported in literature. ${ }^{20}$

N-([1,1':4'1"-terphenyl]-2-yl)acetamide (1i). Prepared from [1,1':4',1'"-terphenyl]-2-amine (S1i), 1i was obtained as a colorless solid (99\% yield). ${ }^{1} \mathrm{H} \operatorname{NMR}\left(\delta, 23{ }^{\circ} \mathrm{C}, 400 \mathrm{MHz}, \mathrm{CDCl}_{3}\right)$ : $8.28(\mathrm{~d}, J=8.3 \mathrm{~Hz}, 1 \mathrm{H}), 7.72(\mathrm{~d}, J=8.2 \mathrm{~Hz}, 2 \mathrm{H}), 7.66(\mathrm{dd}, J=8.2,1.1 \mathrm{~Hz}, 2 \mathrm{H}), 7.51-7.45(\mathrm{~m}$, $4 \mathrm{H}), 7.39(\mathrm{tt}, J=8.0,1.7 \mathrm{~Hz}, 2 \mathrm{H}), 7.29(\mathrm{~d}, J=7.0 \mathrm{~Hz}, 1 \mathrm{H}), 7.21(\mathrm{~d}, J=7.3 \mathrm{~Hz}, 1 \mathrm{H}), 7.18(\mathrm{br}, 1 \mathrm{H})$, $2.06(\mathrm{~s}, 3 \mathrm{H})$. The obtained spectral data are in good agreement with those reported in literature. ${ }^{13}$

N-(5-nitro-[1,1'-biphenyl]-2-yl)acetamide (1j). Prepared from 5-nitro-[1,1'-biphenyl]-2amine (S1j), 1j was obtained as a tan solid (84\% yield). ${ }^{1} \mathrm{H}$ NMR $\left(\delta, 23{ }^{\circ} \mathrm{C}, 400 \mathrm{MHz}, \mathrm{CDCl}_{3}\right)$ : $8.65(\mathrm{~d}, J=9.1 \mathrm{~Hz}, 1 \mathrm{H}), 8.25(\mathrm{dd}, J=9.2,2.7 \mathrm{~Hz}, 1 \mathrm{H}), 8.13(\mathrm{~d}, J=2.7 \mathrm{~Hz}, 1 \mathrm{H}), 7.59-7.52(\mathrm{~m}$, $3 \mathrm{H}), 7.42(\mathrm{br}, 1 \mathrm{H}), 7.41-7.38(\mathrm{~m}, 2 \mathrm{H}), 2.07(\mathrm{~s}, 3 \mathrm{H})$. The obtained spectral data are in good agreement with those reported in literature. ${ }^{21}$

Methyl 6-acetamido-[1,1'-biphenyl]-3-carboxylate (1k). Prepared from 6-amino-[1,1'biphenyl]-3-carboxylate (S1k), 1k was obtained as a colorless solid (85\% yield). ${ }^{1} \mathrm{H}$ NMR $(\delta$, $\left.23{ }^{\circ} \mathrm{C}, 400 \mathrm{MHz}, \mathrm{CDCl}_{3}\right): 8.48(\mathrm{~d}, J=8.2 \mathrm{~Hz}, 1 \mathrm{H}), 8.03(\mathrm{dd}, J=8.7,2.1 \mathrm{~Hz}, 1 \mathrm{H}), 7.92(\mathrm{~d}, J=2.1$ $\mathrm{Hz}, 1 \mathrm{H}), 7.52-7.45$ (m, 3H), 7.38 (dd, $J=8.2,1.4 \mathrm{~Hz}, 2 \mathrm{H}), 7.34$ (br, 1H), 3.90 (s, 3H), 2.04 (s, $3 \mathrm{H})$. The obtained spectral data are in good agreement with those reported in literature.21

N-(4'-(tert-butyl)-[1,1'-biphenyl]-2-yl)acetamide (11). Prepared from 4'-(tert-butyl)-[1,1'biphenyl]-2-amine (S1), 11 was obtained as a colorless solid (69\% yield). ${ }^{1} \mathrm{H}$ NMR $\left(\delta, 23{ }^{\circ} \mathrm{C}\right.$, $400 \mathrm{MHz}, \mathrm{CDCl}_{3}$ ): 8.24 (d, $\left.J=8.2 \mathrm{~Hz}, 1 \mathrm{H}\right), 7.50$ (d, $\left.J=8.2 \mathrm{~Hz}, 2 \mathrm{H}\right), 7.36-7.30(\mathrm{~m}, 3 \mathrm{H}), 7.26-$ $7.23(\mathrm{~m}, 2 \mathrm{H}), 7.16(\mathrm{t}, J=7.4 \mathrm{~Hz}, 1 \mathrm{H}), 2.04(\mathrm{~s}, 3 \mathrm{H}), 1.38(\mathrm{~s}, 9 \mathrm{H})$. The obtained spectral data are in good agreement with those reported in literature. ${ }^{20}$

N-(4'-methoxy-[1,1'-biphenyl]-2-yl)acetamide (1m). Prepared from 4'-methoxy-[1,1'biphenyl]-2-amine (S1m), 1m was obtained as a colorless solid (70\% yield). ${ }^{1} \mathrm{H}$ NMR $(\delta, 23$ $\left.{ }^{\circ} \mathrm{C}, 400 \mathrm{MHz}, \mathrm{CDCl}_{3}\right): 8.28(\mathrm{~d}, J=8.1 \mathrm{~Hz}, 1 \mathrm{H}), 7.39-7.35(\mathrm{~m}, 1 \mathrm{H}), 7.33-7.31(\mathrm{~m}, 2 \mathrm{H}), 7.24(\mathrm{~d}, J$ = $7.3 \mathrm{~Hz}, 1 \mathrm{H}), 7.18(\mathrm{~d}, J=7.5 \mathrm{~Hz}, 1 \mathrm{H}), 7.16(\mathrm{br}, 1 \mathrm{H}), 7.04(\mathrm{~d}, J=8.7 \mathrm{~Hz}, 2 \mathrm{H}), 3.90(\mathrm{~s}, 3 \mathrm{H}), 2.06$ $(\mathrm{s}, 3 \mathrm{H})$. The obtained spectral data are in good agreement with those reported in literature.20

N-(5-methoxyl-[1,1'-biphenyl]-2-yl)acetamide (1n). Prepared from 5-methoxy-[1,1'biphenyl]-2-amine (S1n), 1 n was obtained as a colorless solid (49\% yield). ${ }^{1} \mathrm{H}$ NMR $(\delta, 23$ $\left.{ }^{\circ} \mathrm{C}, 400 \mathrm{MHz}, \mathrm{CDCl}_{3}\right): 7.96(\mathrm{~d}, J=8.9 \mathrm{~Hz}, 1 \mathrm{H}), 7.47(\mathrm{t}, J=7.2 \mathrm{~Hz}, 2 \mathrm{H}), 7.42-7.40(\mathrm{~m}, 1 \mathrm{H}), 7.37-$ $7.35(\mathrm{~m}, 2 \mathrm{H}), 7.03(\mathrm{br}, 1 \mathrm{H}), 6.90(\mathrm{dd}, J=8.9,2.8 \mathrm{~Hz}, 1 \mathrm{H}), 6.81(\mathrm{~d}, J=2.9 \mathrm{~Hz}, 1 \mathrm{H}), 3.81(\mathrm{~s}, 3 \mathrm{H})$, $2.00(\mathrm{~s}, 3 \mathrm{H})$. The obtained spectral data are in good agreement with those reported in literature. ${ }^{20}$

N-(5-methyl-[1,1'-biphenyl]-2-yl)acetamide (10). Prepared from 5-methyl-[1,1'-biphenyl]-2amine (S1o), 10 was obtained as a colorless solid (66\% yield). ${ }^{1} \mathrm{H}$ NMR $\left(\delta, 23{ }^{\circ} \mathrm{C}, 400 \mathrm{MHz}\right.$, 
$\left.\mathrm{CDCl}_{3}\right): 8.11(\mathrm{~d}, J=8.3 \mathrm{~Hz}, 1 \mathrm{H}), 7.50(\mathrm{t}, J=7.3 \mathrm{~Hz}, 2 \mathrm{H}), 7.44-7.43(\mathrm{~m}, 1 \mathrm{H}), 7.41-7.38(\mathrm{~m}, 2 \mathrm{H})$, $7.20(\mathrm{dd}, J=8.3,2.1 \mathrm{~Hz}, 1 \mathrm{H}), 7.09(\mathrm{~s}, 1 \mathrm{H}), 7.06(\mathrm{br}, 1 \mathrm{H}), 2.37(\mathrm{~s}, 3 \mathrm{H}), 2.03(\mathrm{~s}, 3 \mathrm{H})$. The obtained spectral data are in good agreement with those reported in literature. ${ }^{20}$

N-(5-(tert-butyl)-[1,1'-biphenyl]-2-yl)acetamide (1p). Prepared from 5-(tert-butyl)-[1,1'biphenyl]-2-amine (S1p), 1p was obtained as a colorless solid (69\% yield). ${ }^{1} \mathrm{H}$ NMR $\left(\delta, 23^{\circ} \mathrm{C}\right.$, $\left.400 \mathrm{MHz}, \mathrm{CDCl}_{3}\right): 8.12(\mathrm{~d}, J=8.5 \mathrm{~Hz}, 1 \mathrm{H}), 7.49(\mathrm{t}, J=7.3 \mathrm{~Hz}, 2 \mathrm{H}), 7.43-7.38(\mathrm{~m}, 4 \mathrm{H}), 7.24(\mathrm{~d}, J$ $=2.3 \mathrm{~Hz}, 1 \mathrm{H}), 7.04(\mathrm{br}, 1 \mathrm{H}), 2.01(\mathrm{~s}, 3 \mathrm{H}), 1.33(\mathrm{~s}, 9 \mathrm{H})$. The obtained spectral data are in good agreement with those reported in literature. ${ }^{22}$

$\mathrm{N}$-(2'-chloro-[1,1'-biphenyl]-2-yl)acetamide (1q). Prepared from 2'-chloro-[1,1'-biphenyl]2 -amine (S1q), 1q was obtained as an oil (35\% yield). ${ }^{1} \mathrm{H}$ NMR $\left(\delta, 23{ }^{\circ} \mathrm{C}, 400 \mathrm{MHz}, \mathrm{CDCl}_{3}\right)$ : $8.20(\mathrm{~d}, J=8.1 \mathrm{~Hz}, 1 \mathrm{H}), 7.54-7.52(\mathrm{~m}, 1 \mathrm{H}), 7.45-7.30(\mathrm{~m}, 4 \mathrm{H}), 7.19$ (d, $J=6.2 \mathrm{~Hz}, 2 \mathrm{H}), 6.80$ $(\mathrm{br}, 1 \mathrm{H}), 1.99(\mathrm{~s}, 3 \mathrm{H})$. The obtained spectral data are in good agreement with those reported in literature. ${ }^{23}$

N-(4,4"-dichloro-[1,1':3',1"-terphenyl]-2'-yl)acetamide (1r). Prepared from 4,4"-dichloro[1,1':3',1"-terphenyl]-2'-amine (S1r), 1r was obtained as a colorless solid (40\% yield). ${ }^{1} \mathrm{H}$ $\operatorname{NMR}\left(\delta, 23^{\circ} \mathrm{C}, 400 \mathrm{MHz}, \mathrm{CDCl}_{3}\right): 7.44-7.28(\mathrm{~m}, 11 \mathrm{H}), 6.52(\mathrm{~s}, 1 \mathrm{H}), 1.74(\mathrm{~s}, 3 \mathrm{H})$. The obtained spectral data are in good agreement with those reported in literature. ${ }^{24}$

N-(4,4"-difluoro-[1,1':3',1"-terphenyl]-2'-yl)acetamide (1s). Prepared from 4,4"-difluoro[1,1':3',1"-terphenyl]-2'-amine (S1s), 1s was obtained as a colorless solid (45\% yield). ${ }^{1} \mathrm{H}$ $\operatorname{NMR}\left(\delta, 23^{\circ} \mathrm{C}, 400 \mathrm{MHz}, \mathrm{CDCl}_{3}\right): 7.42-7.32(\mathrm{~m}, 7 \mathrm{H}), 7.09(\mathrm{t}, J=8.7 \mathrm{~Hz}, 4 \mathrm{H}), 6.47(\mathrm{~s}, 1 \mathrm{H}), 1.73$ $(\mathrm{s}, 3 \mathrm{H})$. The obtained spectral data are in good agreement with those reported in literature. ${ }^{24}$

$\mathrm{N}-(4$, '5-dichloro-[1,1'-biphenyl]-2-yl)acetamide (1t). Prepared from 4',5-dichloro-[1,1'biphenyl]-2-amine (S1t), 1t was obtained as a colorless solid (66\% yield). ${ }^{1} \mathrm{H}$ NMR $\left(\delta, 23^{\circ} \mathrm{C}\right.$, $\left.400 \mathrm{MHz}, \mathrm{CDCl}_{3}\right): 8.19(\mathrm{~d}, J=8.9 \mathrm{~Hz}, 1 \mathrm{H}), 7.49-7.46(\mathrm{~m}, 2 \mathrm{H}), 7.35-7.28(\mathrm{~m}, 3 \mathrm{H}), 7.20(\mathrm{~d}, J=$ $2.3 \mathrm{~Hz}, 1 \mathrm{H}), 6.94-6.94(\mathrm{~m}, 1 \mathrm{H}), 2.03(\mathrm{~s}, 3 \mathrm{H})$. The obtained spectral data are in good agreement with those reported in literature..$^{25}$

N-(4'-chloro-5-fluoro-[1,1'-biphenyl]-2-yl)acetamide (1u). Prepared from 4'-chloro-5-fluoro[1,1'-biphenyl]-2-amine (S1 u), $\mathbf{1} \mathbf{u}$ was obtained as a colorless solid (61\% yield). ${ }^{1} \mathrm{H}$ NMR $(\delta$, $\left.23{ }^{\circ} \mathrm{C}, 400 \mathrm{MHz}, \mathrm{CDCl}_{3}\right): 8.11-8.07(\mathrm{~m}, 1 \mathrm{H}), 7.47(\mathrm{~d}, J=8.4 \mathrm{~Hz}, 2 \mathrm{H}), 7.30(\mathrm{~d}, J=8.4 \mathrm{~Hz}, 2 \mathrm{H})$, $7.08(\mathrm{td}, J=8.5,2.9 \mathrm{~Hz}, 1 \mathrm{H}), 6.94(\mathrm{dd}, J=8.9,3.0 \mathrm{~Hz}, 1 \mathrm{H}), 6.87(\mathrm{~s}, 1 \mathrm{H}), 2.03(\mathrm{~s}, 3 \mathrm{H}) .{ }^{13} \mathrm{C} \mathrm{NMR}$ $\left(\delta, 23{ }^{\circ} \mathrm{C}, 100 \mathrm{MHz}, \mathrm{CDCl}_{3}\right): 168.5,135.8,134.8,130.7,130.5,129.6,124.84,124.76,116.9$, 116.6, 115.6, 115.4, 24.5. HRMS-ESI: calculated for $[\mathrm{M}+\mathrm{H}]=264.0586$, observed $[\mathrm{M}+\mathrm{H}]=$ 264.0584 .

$\mathrm{N}$-(2-bromo-4-formylphenyl)acetamide (S1v). Prepared from 4-amino-3bromobenzaldehyde, $\mathbf{S 1 v}$ was obtained as a colorless solid (50\% yield). ${ }^{1} \mathrm{H}$ NMR $\left(\delta, 23^{\circ} \mathrm{C}\right.$, $\left.400 \mathrm{MHz}, \mathrm{CDCl}_{3}\right): 9.88(\mathrm{~s}, 1 \mathrm{H}), 8.64(\mathrm{~d}, J=8.5 \mathrm{~Hz}, 1 \mathrm{H}), 8.09(\mathrm{~d}, J=1.8 \mathrm{~Hz}, 1 \mathrm{H}), 7.86(\mathrm{br}, 1 \mathrm{H})$, $7.82(\mathrm{dd}, J=8.5,1.8 \mathrm{~Hz}, 1 \mathrm{H}), 2.30(\mathrm{~s}, 3 \mathrm{H})$. The obtained spectral data are in good agreement with those reported in literature..$^{25}$ 


\section{Synthesis of $N$-(5-formyl-4'-methoxy-[1,1'-biphenyl]-2-yl)acetamide (1v)}

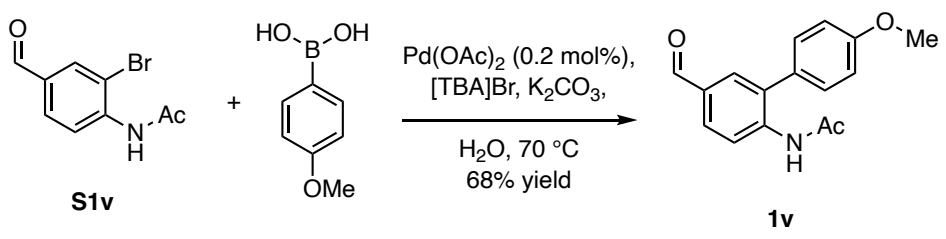

A 20 -mL scintillation vial was charged with $\mathrm{N}$-(2-bromo-4-formylphenyl)acetamide (S1v, $600 \mathrm{mg}, 2.49 \mathrm{mmol}, 1.00$ equiv.), 4-methoxyphenylboronic acid (568 mg, $3.74 \mathrm{mmol}, 1.50$ equiv.), $\mathrm{Pd}(\mathrm{OAc})_{2}(1.3 \mathrm{mg}, 6.0 \mu \mathrm{mol}, 0.20 \mathrm{~mol} \%$ ), [TBA]Br (903 mg, $2.90 \mathrm{mmol}, 1.00$ equiv.), $\mathrm{K}_{2} \mathrm{CO}_{3}$ (977 mg, $7.07 \mathrm{mmol}, 2.52$ equiv.), and distilled water $(4 \mathrm{~mL}$ ) and was fitted with a rubber septum. The reaction vessel was purged with $\mathrm{N}_{2}$ for 10 min after which time the reaction mixture was heated at $70^{\circ} \mathrm{C}$ for $16 \mathrm{~h}$. The reaction mixture was cooled to $23^{\circ} \mathrm{C}$ and was then extracted with ethyl acetate $(3 \times 10 \mathrm{~mL})$. The combined organic fractions were dried over $\mathrm{MgSO}_{4}$ and solvent was removed under reduced pressure. The obtained residue was purified by $\mathrm{SiO}_{2}$ gel chromatography (eluent 95:5 hexanes:ethyl acetate) to afford 458 mg of the title compound ( $68 \%$ yield). ${ }^{1} \mathrm{H}$ NMR $\left(\delta, 23{ }^{\circ} \mathrm{C}, 400 \mathrm{MHz}, \mathrm{CDCl}_{3}\right): 9.94(\mathrm{~s}, 1 \mathrm{H}), 8.60$ $(\mathrm{d}, J=8.4 \mathrm{~Hz}, 1 \mathrm{H}), 7.85(\mathrm{dd}, J=8.5,2.0 \mathrm{~Hz}, 1 \mathrm{H}), 7.74(\mathrm{~d}, J=2.0 \mathrm{~Hz}, 1 \mathrm{H}), 7.42(\mathrm{br}, 1 \mathrm{H}), 7.31(\mathrm{~d}$, $J=8.8 \mathrm{~Hz}, 2 \mathrm{H}), 7.05(\mathrm{~d}, J=8.8 \mathrm{~Hz}, 2 \mathrm{H}), 3.89(\mathrm{~s}, 3 \mathrm{H}), 2.07(\mathrm{~s}, 3 \mathrm{H}) .{ }^{13} \mathrm{C}$ NMR $\left(\delta, 23{ }^{\circ} \mathrm{C}, 100 \mathrm{MHz}\right.$, $\mathrm{CDCl}_{3}$ ): 191.3, 168.5, 160.0, 140.7, 132.1, 131.4, 130.8, 130.5, 128.7, 120.3, 115.1, 55.6, 25.1. HRMS-ESI: calculated for $[\mathrm{M}+\mathrm{H}]=270.1125$, observed $[\mathrm{M}+\mathrm{H}]=270.1116$. 


\section{B.2 Intramolecular C-H Amination Electrocatalysis}

\section{General Procedure for Intramolecular Amination Reactions}

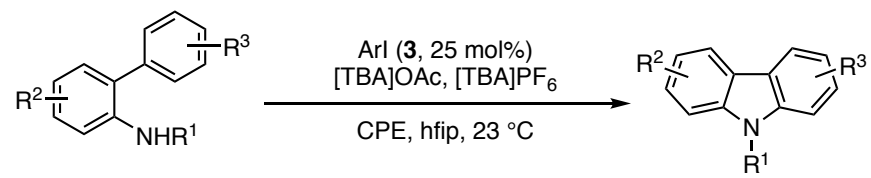

Constant Potential Conditions. A 10 -mL glass vial was charged with $\mathrm{N}$-arylacetamide $(\mathbf{1}$, $0.201 \mathrm{mmol}, 1.00$ equiv.), 4-iodoanisole (3a, $12.0 \mathrm{mg}, 0.0512 \mathrm{mmol}, 25.3 \mathrm{~mol} \%$ ), tetrabutylammonium acetate $(121 \mathrm{mg}, 0.401 \mathrm{mmol}, 1.99$ equiv.), tetrabutylammonium hexafluorophosphate (390 mg, $1.01 \mathrm{mmol}, 5.02$ equiv.), and hfip (5.0 mL) and was fitted with glassy carbon anode, platinum cathode, and $\mathrm{Ag}^{+} / \mathrm{Ag}$ reference electrode. A constant potential of $1.5 \mathrm{~V}$ vs. $\mathrm{Ag}^{+} / \mathrm{Ag}$ was applied to the reaction mixture with stirring at $23{ }^{\circ} \mathrm{C}$ until $\sim 80 \mathrm{C}$ charge $(\sim 4.15 \mathrm{~F} / \mathrm{mol})$ is passed. The solvent was removed under reduced pressure. The obtained residue was purified by $\mathrm{SiO}_{2}$ gel chromatography (eluent 95:5 hexanes:ethyl acetate) to afford the $\mathrm{N}$-acyl carbazole product indicated below.

Constant Current Conditions. A 10 -mL glass vial was charged with $\mathrm{N}$-arylacetamide $(0.201$ mmol, 1.00 equiv.), aryliodide catalyst (3, $0.0512 \mathrm{mmol}, 25.3 \mathrm{~mol} \%)$, tetrabutylammonium acetate (121 mg, $0.401 \mathrm{mmol}, 1.99$ equiv.), and tetrabutylammonium hexafluorophosphate (390 mg, $1.01 \mathrm{mmol}, 5.02$ equiv.), and hfip (5.0 mL) and was fitted with glassy carbon anode and platinum cathode. A constant current of $5 \mathrm{~mA}$ was applied to the reaction mixture with stirring at $23{ }^{\circ} \mathrm{C}$ until $4.00 \mathrm{~F} / \mathrm{mol}$ is passed. The solvent was removed under reduced pressure. The obtained residue was purified by $\mathrm{SiO}_{2}$ gel chromatography (eluent 95:5 hexanes:ethyl acetate) to afford the $N$-acyl carbazole product indicated below.

1-(9H-carbazol-9-yl)ethan-1-one (2a). Prepared under constant potential conditions using 3a as catalyst. Yield: $32 \mathrm{mg}\left(76 \%\right.$ yield). ${ }^{1} \mathrm{H}$ NMR $\left(\delta, 23^{\circ} \mathrm{C}, 400 \mathrm{MHz}, \mathrm{CDCl}_{3}\right):$ ): $8.15(\mathrm{~d}, J=8.3$ $\mathrm{Hz}, 2 \mathrm{H}), 8.02(\mathrm{~d}, J=6.7 \mathrm{~Hz}, 2 \mathrm{H}), 7.51(\mathrm{t}, J=7.1 \mathrm{~Hz}, 2 \mathrm{H}), 7.44(\mathrm{t}, J=7.5 \mathrm{~Hz}, 2 \mathrm{H}), 2.90(\mathrm{~s}, 3 \mathrm{H})$. The obtained spectral data are in good agreement with those reported in literature. 26

1-(3-Bromo-9H-carbazol-9-yl)ethan-1-one (2b). Prepared under constant potential conditions using 3 a as catalyst. Yield: $45 \mathrm{mg}\left(78 \%\right.$ yield). ${ }^{1} \mathrm{H} \mathrm{NMR}\left(\delta, 23^{\circ} \mathrm{C}, 400 \mathrm{MHz}, \mathrm{CDCl}_{3}\right)$ : $8.20(\mathrm{~d}, J=8.9 \mathrm{~Hz}, 1 \mathrm{H}), 8.12-8.10(\mathrm{~m}, 2 \mathrm{H}), 7.96(\mathrm{~d}, J=7.7 \mathrm{~Hz}, 1 \mathrm{H}), 7.57(\mathrm{~d}, J=8.9 \mathrm{~Hz}, 1 \mathrm{H}), 7.52$ $(\mathrm{d}, J=8.5 \mathrm{~Hz}, 1 \mathrm{H}), 7.41(\mathrm{t}, J=7.5 \mathrm{~Hz}, 1 \mathrm{H}), 2.88(\mathrm{~s}, 3 \mathrm{H})$. The obtained spectral data are in good agreement with those reported in literature. ${ }^{26}$

1-(3-Chloro-9H-carbazol-9-yl)ethan-1-one (2c). Prepared under constant potential conditions using $3 \mathrm{a}$ as catalyst. Yield: $39 \mathrm{mg}\left(80 \%\right.$ yield). ${ }^{1} \mathrm{H} \mathrm{NMR}\left(\delta, 23^{\circ} \mathrm{C}, 400 \mathrm{MHz}, \mathrm{CDCl}_{3}\right)$ : $8.26(\mathrm{~d}, J=8.9 \mathrm{~Hz}, 1 \mathrm{H}), 8.12(\mathrm{~d}, J=8.4 \mathrm{~Hz}, 1 \mathrm{H}), 7.98-7.95(\mathrm{~m}, 2 \mathrm{H}), 7.54(\mathrm{td}, J=7.9,1.3 \mathrm{~Hz}, 1 \mathrm{H})$, 7.46-7.40 (m, $2 \mathrm{H}), 2.89(\mathrm{~s}, 3 \mathrm{H})$. The obtained spectral data are in good agreement with those reported in literature. ${ }^{26}$

1-(3-Fluoro-9H-carbazol-9-yl)ethan-1-one (2d). Prepared under constant potential conditions using 3a as catalyst. Yield: $29 \mathrm{mg}\left(64 \%\right.$ yield). ${ }^{1} \mathrm{H} \mathrm{NMR}\left(\delta, 23^{\circ} \mathrm{C}, 400 \mathrm{MHz}, \mathrm{CDCl}_{3}\right)$ : 8.33-8.30 (m, 1H), $8.11(\mathrm{~d}, J=8.4 \mathrm{~Hz}, 1 \mathrm{H}), 7.97$ (ddd, $J=7.7,1.4,0.7 \mathrm{~Hz}, 1 \mathrm{H}), 7.66-7.64(\mathrm{~m}$, 1H), 7.52 (ddd, $J=8.5,7.3,1.3 \mathrm{~Hz}, 1 \mathrm{H}$ ), 7.41 (td, $J=7.5,0.9 \mathrm{~Hz}, 1 \mathrm{H}), 7.20$ (td, $J=9.0,2.7 \mathrm{~Hz}$, 
1H), $2.90(\mathrm{~s}, 3 \mathrm{H})$. The obtained spectral data are in good agreement with those reported in literature. ${ }^{27}$

1-(2-Bromo-9H-carbazol-9-yl)ethan-1-one (2e). Prepared under constant potential conditions using 3a as catalyst. Yield: $43 \mathrm{mg}$ (75\% yield). ${ }^{1} \mathrm{H}$ NMR $\left(\delta, 23{ }^{\circ} \mathrm{C}, 400 \mathrm{MHz}, \mathrm{CDCl}_{3}\right)$ : $8.52(\mathrm{~d}, J=1.5 \mathrm{~Hz}, 1 \mathrm{H}), 8.08(\mathrm{~d}, J=8.4 \mathrm{~Hz}, 1 \mathrm{H}), 7.97(\mathrm{~d}, J=7.7 \mathrm{~Hz}, 1 \mathrm{H}), 7.83(\mathrm{~d}, J=8.2 \mathrm{~Hz}, 1 \mathrm{H})$, $7.51(\mathrm{~d}, J=8.4 \mathrm{~Hz}, 2 \mathrm{H}), 7.40(\mathrm{t}, J=7.5 \mathrm{~Hz}, 1 \mathrm{H}), 2.88(\mathrm{~s}, 3 \mathrm{H}) .{ }^{13} \mathrm{C}$ NMR $\left(\delta, 23{ }^{\circ} \mathrm{C}, 100 \mathrm{MHz}\right.$, $\mathrm{CDCl}_{3}$ ): 170.0, 139.6, 138.6, 127.8, 127.1, 125.9, 125.3, 124.0, 121.1, 120.8, 120.2, 119.9, 116.0, 27.9. HRMS-ESI: calculated for $[\mathrm{M}+\mathrm{H}]=288.0019$, observed $[\mathrm{M}+\mathrm{H}]=288.0011$.

1-(2-Chloro-9H-carbazol-9-yl)ethan-1-one (2f). Prepared under constant potential conditions using 3a as catalyst. Yield: $30 \mathrm{mg}\left(61 \%\right.$ yield). ${ }^{1} \mathrm{H} \mathrm{NMR}\left(\delta, 23{ }^{\circ} \mathrm{C}, 400 \mathrm{MHz}, \mathrm{CDCl}_{3}\right)$ : $8.38(\mathrm{~s}, 1 \mathrm{H}), 8.10(\mathrm{~d}, J=8.4 \mathrm{~Hz}, 1 \mathrm{H}), 7.98(\mathrm{~d}, J=7.0 \mathrm{~Hz}, 1 \mathrm{H}), 7.90(\mathrm{~d}, J=8.3 \mathrm{~Hz}, 1 \mathrm{H}), 7.50(\mathrm{t}, J$ $=7.2 \mathrm{~Hz}, 1 \mathrm{H}), 7.43-7.37(\mathrm{~m}, 2 \mathrm{H}), 2.89(\mathrm{~s}, 3 \mathrm{H})$. The obtained spectral data are in good agreement with those reported in literature. ${ }^{26}$

1-(2-Fluoro-9H-carbazol-9-yl)ethan-1-one (2g). Prepared under constant potential conditions using $3 a$ as catalyst. Yield: $36 \mathrm{mg}$ (78\% yield). ${ }^{1} \mathrm{H} \operatorname{NMR}\left(\delta, 23{ }^{\circ} \mathrm{C}, 400 \mathrm{MHz}, \mathrm{CDCl}_{3}\right)$ : $8.09(\mathrm{td}, J=6.6,3.0 \mathrm{~Hz}, 2 \mathrm{H}), 7.97-7.91(\mathrm{~m}, 2 \mathrm{H}), 7.47(\mathrm{ddd}, J=8.5,7.2,1.3 \mathrm{~Hz}, 1 \mathrm{H}), 7.40(\mathrm{td}, J$ $=7.5,1.0 \mathrm{~Hz}, 1 \mathrm{H}), 7.14(\mathrm{td}, J=8.7,2.3 \mathrm{~Hz}, 1 \mathrm{H}), 2.89(\mathrm{~s}, 3 \mathrm{H})$. The obtained spectral data are in good agreement with those reported in literature. ${ }^{28}$

9-Acetyl-9H-carbazole-3-carbaldehyde (2h). Prepared under constant potential conditions using 3a as catalyst. Yield: $20 \mathrm{mg}$ (48\% yield). ${ }^{1} \mathrm{H}$ NMR $\left(\delta, 23{ }^{\circ} \mathrm{C}, 400 \mathrm{MHz}, \mathrm{CDCl}_{3}\right): 10.15(\mathrm{~s}$, $1 \mathrm{H}), 8.44(\mathrm{dd}, J=1.7,0.5 \mathrm{~Hz}, 1 \mathrm{H}), 8.30(\mathrm{~d}, J=8.6 \mathrm{~Hz}, 1 \mathrm{H}), 7.98-7.94(\mathrm{~m}, 2 \mathrm{H}), 7.82(\mathrm{~d}, J=2.2$ $\mathrm{Hz}, 1 \mathrm{H}), 7.07(\mathrm{dd}, J=8.6,2.2 \mathrm{~Hz}, 1 \mathrm{H}), 3.97(\mathrm{~s}, 3 \mathrm{H}), 2.93(\mathrm{~s}, 3 \mathrm{H})$. The obtained spectral data are in good agreement with those reported in literature. ${ }^{26}$

1-(2-Phenyl-9H-carbazol-9-yl)ethan-1-one (2i). Prepared under constant current conditions using 3a as catalyst. Yield: $48 \mathrm{mg}\left(84 \%\right.$ yield). ${ }^{1} \mathrm{H}$ NMR $\left(\delta, 23{ }^{\circ} \mathrm{C}, 400 \mathrm{MHz}, \mathrm{CDCl}_{3}\right): 8.54(\mathrm{~d}, J$ $=0.7 \mathrm{~Hz}, 1 \mathrm{H}), 8.22(\mathrm{~d}, J=8.3 \mathrm{~Hz}, 1 \mathrm{H}), 8.09-8.04(\mathrm{~m}, 2 \mathrm{H}), 7.73(\mathrm{dd}, J=8.3,1.1 \mathrm{~Hz}, 2 \mathrm{H}), 7.67$ $(\mathrm{dd}, J=8.0,1.4 \mathrm{~Hz}, 1 \mathrm{H}), 7.55-7.50(\mathrm{~m}, 3 \mathrm{H}), 7.46-7.40(\mathrm{~m}, 2 \mathrm{H}), 2.97(\mathrm{~s}, 3 \mathrm{H})$. The obtained spectral data are in good agreement with those reported in literature. ${ }^{29}$

1-(3-Nitro-9H-carbazol-9-yl)ethan-1-one (2j). Prepared under constant current conditions using 3a as catalyst. Yield: $22 \mathrm{mg}\left(43 \%\right.$ yield). ${ }^{1} \mathrm{H}$ NMR $\left(\delta, 23{ }^{\circ} \mathrm{C}, 400 \mathrm{MHz}, \mathrm{CDCl}_{3}\right): 8.90(\mathrm{~d}, J$ $=2.2 \mathrm{~Hz}, 1 \mathrm{H}), 8.54(\mathrm{~d}, J=9.2 \mathrm{~Hz}, 1 \mathrm{H}), 8.41(\mathrm{dd}, J=9.2,2.4 \mathrm{~Hz}, 1 \mathrm{H}), 8.15-8.10(\mathrm{~m}, 2 \mathrm{H}), 7.63$ $(\mathrm{td}, J=7.9,1.3 \mathrm{~Hz}, 1 \mathrm{H}), 7.54-7.50(\mathrm{~m}, 1 \mathrm{H}), 2.97(\mathrm{~s}, 3 \mathrm{H})$. The obtained spectral data are in good agreement with those reported in literature. ${ }^{30}$

Methyl 9-acetyl-9H-carbazole-3-carboxylate (2k). Prepared under constant potential conditions using $\mathbf{3 b}$ as catalyst. CPE carried out $1.9 \mathrm{~V}$ vs. $\mathrm{Ag}^{+} / \mathrm{Ag}$ with hfip/ $\mathrm{CH}_{2} \mathrm{Cl}_{2}(5 / 1)$ solvent mixture. Yield: $38 \mathrm{mg}\left(71 \%\right.$ yield). ${ }^{1} \mathrm{H} \operatorname{NMR}\left(\delta, 23{ }^{\circ} \mathrm{C}, 400 \mathrm{MHz}, \mathrm{CDCl}_{3}\right): 8.73(\mathrm{dd}, J=$ 1.8, $0.6 \mathrm{~Hz}, 1 \mathrm{H}), 8.35(\mathrm{dd}, J=8.8,0.5 \mathrm{~Hz}, 1 \mathrm{H}), 8.22-8.19(\mathrm{~m}, 2 \mathrm{H}), 8.11(\mathrm{ddd}, J=7.7,1.4,0.7 \mathrm{~Hz}$, $1 \mathrm{H}), 7.56$ (ddd, $J=8.5,7.2,1.3 \mathrm{~Hz}, 1 \mathrm{H}$ ), 7.47 (td, $J=7.5,0.9 \mathrm{~Hz}, 1 \mathrm{H}), 4.02(\mathrm{~s}, 3 \mathrm{H}), 2.95$ (s, $3 \mathrm{H})$.The obtained spectral data are in good agreement with those reported in literature. ${ }^{31}$

1-(2-Tert-butyl-9H-carbazol-9-yl)ethan-1-one (2l). Prepared under constant potential conditions using 3a as catalyst. Yield: $34 \mathrm{mg}\left(65 \%\right.$ yield). ${ }^{1} \mathrm{H} \mathrm{NMR}\left(\delta, 23{ }^{\circ} \mathrm{C}, 400 \mathrm{MHz}, \mathrm{CDCl}_{3}\right)$ : $8.33(\mathrm{~s}, 1 \mathrm{H}), 8.15(\mathrm{~d}, J=8.3 \mathrm{~Hz}, 1 \mathrm{H}), 7.97(\mathrm{~d}, J=8.3 \mathrm{~Hz}, 1 \mathrm{H}), 7.91(\mathrm{~d}, J=7.6 \mathrm{~Hz}, 1 \mathrm{H}), 7.45(\mathrm{t}, J$ 
$=9.2 \mathrm{~Hz}, 2 \mathrm{H}), 7.37(\mathrm{t}, J=7.5 \mathrm{~Hz}, 1 \mathrm{H}), 2.90(\mathrm{~s}, 3 \mathrm{H}), 1.44(\mathrm{~s}, 10 \mathrm{H})$. The obtained spectral data are in good agreement with those reported in literature. ${ }^{29}$

1-(2-Methoxy-9H-carbazol-9-yl)ethan-1-one (2m). Prepared under constant potential conditions using 3a as catalyst. Yield: $21 \mathrm{mg}$ (44\% yield). ${ }^{1} \mathrm{H} \mathrm{NMR}\left(\delta, 23{ }^{\circ} \mathrm{C}, 400 \mathrm{MHz}, \mathrm{CDCl}_{3}\right)$ : $8.05(\mathrm{~d}, J=7.8 \mathrm{~Hz}, 1 \mathrm{H}), 7.93-7.89(\mathrm{~m}, 2 \mathrm{H}), 7.87-7.85(\mathrm{~m}, 1 \mathrm{H}), 7.42-7.34(\mathrm{~m}, 2 \mathrm{H}), 6.99(\mathrm{dd}, J$ $=8.5,2.3 \mathrm{~Hz}, 1 \mathrm{H}), 3.93(\mathrm{~s}, 3 \mathrm{H}), 2.88(\mathrm{~s}, 3 \mathrm{H})$. The obtained spectral data are in good agreement with those reported in literature 26

1-(3-Methoxy-9H-carbazol-9-yl)ethan-1-one (2n). Prepared under constant potential conditions using 3a as catalyst. Yield: $4.8 \mathrm{mg}$ (10\% yield). ${ }^{1} \mathrm{H}$ NMR $\left(\delta, 23{ }^{\circ} \mathrm{C}, 400 \mathrm{MHz}, \mathrm{CDCl}_{3}\right)$ : 7.91-7.89 (m, 2H), 7.52-7.48 (m, 2H), $7.39(\mathrm{~d}, J=7.4 \mathrm{~Hz}, 1 \mathrm{H}), 7.08(\mathrm{~d}, J=2.4 \mathrm{~Hz}, 1 \mathrm{H}), 7.00(\mathrm{~d}$, $J=2.4 \mathrm{~Hz}, 1 \mathrm{H}), 3.90(\mathrm{~s}, 3 \mathrm{H}), 2.63(\mathrm{~s}, 3 \mathrm{H})$. The obtained spectral data are in good agreement with those reported in literature. ${ }^{28}$

1-(3-Methyl-9H-carbazol-9-yl)ethan-1-one (2o). Prepared under constant potential conditions using $\mathbf{3 b}$ as catalyst. CPE carried out $1.9 \mathrm{~V}$ vs. $\mathrm{Ag}^{+} / \mathrm{Ag}$ with hfip/ $\mathrm{CH}_{2} \mathrm{Cl}_{2}(5 / 1)$ solvent mixture. Yield: $4.4 \mathrm{mg}\left(10 \%\right.$ yield). ${ }^{1} \mathrm{H} \mathrm{NMR}\left(\delta, 23{ }^{\circ} \mathrm{C}, 400 \mathrm{MHz}, \mathrm{CDCl}_{3}\right): 8.23(\mathrm{~d}, J=$ $8.4 \mathrm{~Hz}, 1 \mathrm{H}$ ), 8.08 (d, $J=8.5 \mathrm{~Hz}, 1 \mathrm{H}), 7.97$ (ddd, $J=7.7,1.4,0.6 \mathrm{~Hz}, 1 \mathrm{H}$ ), 7.80 (t, $J=0.9 \mathrm{~Hz}, 1 \mathrm{H}$ ), $7.49-7.45(\mathrm{~m}, 1 \mathrm{H}), 7.38$ (td, $J=7.5,1.0 \mathrm{~Hz}, 1 \mathrm{H}), 7.30$ (ddd, $J=8.5,1.8,0.5 \mathrm{~Hz}, 1 \mathrm{H}), 2.88(\mathrm{~s}, 3 \mathrm{H})$, $2.53(\mathrm{~s}, 3 \mathrm{H})$. The obtained spectral data are in good agreement with those reported in literature. ${ }^{32}$

1-(3-Tert-butyl-9H-carbazol-9-yl)ethan-1-one (2p). Prepared under constant potential conditions using 3a as catalyst. Yield: $36 \mathrm{mg}$ (65\% yield). ${ }^{1} \mathrm{H} \mathrm{NMR}\left(\delta, 23{ }^{\circ} \mathrm{C}, 400 \mathrm{MHz}, \mathrm{CDCl}_{3}\right)$ : $8.27(\mathrm{~d}, J=8.3 \mathrm{~Hz}, 1 \mathrm{H}), 8.10(\mathrm{~d}, J=8.8 \mathrm{~Hz}, 1 \mathrm{H}), 8.04-8.01(\mathrm{~m}, 2 \mathrm{H}), 7.55(\mathrm{dd}, J=8.8,2.1 \mathrm{~Hz}$, 1H), 7.48 (ddd, $J=8.4,7.2,1.3 \mathrm{~Hz}, 1 \mathrm{H}), 7.40$ (td, $J=7.5,0.9 \mathrm{~Hz}, 1 \mathrm{H}), 2.89(\mathrm{~s}, 3 \mathrm{H}), 1.46(\mathrm{~s}, 9 \mathrm{H})$. The obtained spectral data are in good agreement with those reported in literature. ${ }^{27}$

1-(4-Chloro-9H-carbazol-9-yl)ethan-1-one (2q). Prepared under constant potential conditions using 3a as catalyst. Yield: $41 \mathrm{mg}$ (85\% yield). ${ }^{1} \mathrm{H}$ NMR $\left(\delta, 23{ }^{\circ} \mathrm{C}, 400 \mathrm{MHz}, \mathrm{CDCl}_{3}\right)$ : $8.68(\mathrm{dt}, J=7.9,0.6 \mathrm{~Hz}, 1 \mathrm{H}), 8.25(\mathrm{dd}, J=7.0,2.1 \mathrm{~Hz}, 1 \mathrm{H}), 8.17(\mathrm{~d}, J=8.5 \mathrm{~Hz}, 1 \mathrm{H}), 7.54-7.52$ $(\mathrm{m}, 1 \mathrm{H}), 7.46-7.36(\mathrm{~m}, 3 \mathrm{H}), 2.90(\mathrm{~s}, 3 \mathrm{H}) .{ }^{13} \mathrm{C}$ NMR $\left(\delta, 23{ }^{\circ} \mathrm{C}, 125 \mathrm{MHz}, \mathrm{CDCl}_{3}\right): 170.2,140.2$, 138.8, 128.7, 127.9, 127.6, 125.4, 125.1, 123.9, 123.50, 123.41, 115.6, 114.8, 28.1. HRMS-ESI: calculated for $[\mathrm{M}+\mathrm{H}]=244.0524$, observed $[\mathrm{M}+\mathrm{H}]=244.0521$.

N-(4,4"-dichloro-[1,1':3',1"-terphenyl]-2'-yl)acetamide (2r). Prepared under constant potential conditions using $\mathbf{3 b}$ as catalyst. CPE carried out $1.9 \mathrm{~V}$ vs. $\mathrm{Ag}+\mathrm{Ag}$ with hfip $/ \mathrm{CH}_{2} \mathrm{Cl}_{2}$ (5/1) solvent mixture. Yield: $38 \mathrm{mg}\left(53 \%\right.$ yield). ${ }^{1} \mathrm{H} \mathrm{NMR}\left(\delta, 23{ }^{\circ} \mathrm{C}, 400 \mathrm{MHz}, \mathrm{CDCl}_{3}\right): 8.24(\mathrm{~d}$, $J=1.9 \mathrm{~Hz}, 1 \mathrm{H}), 7.97(\mathrm{dd}, J=7.5,1.3 \mathrm{~Hz}, 1 \mathrm{H}), 7.91(\mathrm{~d}, J=8.3 \mathrm{~Hz}, 1 \mathrm{H}), 7.54-7.49(\mathrm{~m}, 6 \mathrm{H}), 7.38$ $(\mathrm{dd}, J=8.2,1.8 \mathrm{~Hz}, 1 \mathrm{H}), 1.82(\mathrm{~s}, 4 \mathrm{H})$. The obtained spectral data are in good agreement with those reported in literature. ${ }^{24}$

N-(4,4"-difluoro-[1,1':3',1'--terphenyl]-2'-yl)acetamide (2s). Prepared under constant potential conditions using $\mathbf{3 b}$ as catalyst. CPE carried out $1.9 \mathrm{~V}$ vs. $\mathrm{Ag}+\mathrm{Ag}$ with $\mathrm{hfip} / \mathrm{CH}_{2} \mathrm{Cl}_{2}$ (5/1) solvent mixture. Yield: $35 \mathrm{mg}$ (55\% yield). ${ }^{1} \mathrm{H}$ NMR $\left(\delta, 23{ }^{\circ} \mathrm{C}, 400 \mathrm{MHz}, \mathrm{CDCl}_{3}\right): 7.96-$ $7.93(\mathrm{~m}, 3 \mathrm{H}), 7.57(\mathrm{dd}, J=8.8,5.3 \mathrm{~Hz}, 2 \mathrm{H}), 7.47(\mathrm{~d}, J=7.6 \mathrm{~Hz}, 1 \mathrm{H}), 7.42(\mathrm{~d}, J=1.2 \mathrm{~Hz}, 1 \mathrm{H})$, $7.23-7.13(\mathrm{~m}, 3 \mathrm{H}), 1.79(\mathrm{~s}, 3 \mathrm{H})$. The obtained spectral data are in good agreement with those reported in literature. ${ }^{24}$ 
1-(2,6-Dichloro-9H-carbazol-9-yl)ethan-1-one (2t). Prepared under constant potential conditions using $3 \mathrm{a}$ as catalyst. Yield: $47 \mathrm{mg}\left(84 \%\right.$ yield). ${ }^{1} \mathrm{H} \mathrm{NMR}\left(\delta, 23^{\circ} \mathrm{C}, 400 \mathrm{MHz}, \mathrm{CDCl}_{3}\right)$ : $8.24(\mathrm{~d}, J=1.7 \mathrm{~Hz}, 1 \mathrm{H}), 8.11$ (d, $J=8.9 \mathrm{~Hz}, 1 \mathrm{H}), 7.90-7.83(\mathrm{~m}, 2 \mathrm{H}), 7.41$ (ddd, $J=21.6,8.6,2.0$ $\mathrm{Hz}, 2 \mathrm{H}), 2.86(\mathrm{~s}, 3 \mathrm{H}) .{ }^{13} \mathrm{C} \mathrm{NMR}\left(\delta, 23{ }^{\circ} \mathrm{C}, 100 \mathrm{MHz}, \mathrm{CDCl}_{3}\right): 169.7,139.6,137.2,134.0,129.8$, $127.7,127.2,124.6,123.9,120.9,119.8,117.4,116.9,27.8$. HRMS-ESI: calculated for $[\mathrm{M}+\mathrm{H}]=$ 278.0134, observed $[\mathrm{M}+\mathrm{H}]=278.0132$.

1-(2-Chloro-6-fluoro-9H-carbazol-9-yl)ethan-1-one (2u). Prepared under constant potential conditions using 3a as catalyst. Yield: $37 \mathrm{mg}\left(72 \%\right.$ yield). ${ }^{1} \mathrm{H} \mathrm{NMR}\left(\delta, 23^{\circ} \mathrm{C}, 400 \mathrm{MHz}, \mathrm{CDCl}_{3}\right)$ : $8.24(\mathrm{~s}, 1 \mathrm{H}), 8.17(\mathrm{dd}, J=9.2,4.3 \mathrm{~Hz}, 1 \mathrm{H}), 7.86(\mathrm{~d}, J=8.3 \mathrm{~Hz}, 1 \mathrm{H}), 7.62-7.60(\mathrm{~m}, 1 \mathrm{H}), 7.39$ (dd, $J=8.3,1.8 \mathrm{~Hz}, 1 \mathrm{H}), 7.21(\mathrm{td}, J=9.0,2.7 \mathrm{~Hz}, 1 \mathrm{H}), 2.88(\mathrm{~s}, 3 \mathrm{H}) .{ }^{13} \mathrm{C} \mathrm{NMR}\left(\delta, 23{ }^{\circ} \mathrm{C}, 100 \mathrm{MHz}\right.$, $\left.\mathrm{CDCl}_{3}\right): 169.5,133.8,124.3,120.8,117.5,116.8,115.0,114.8,27.6$. HRMS-ESI: calculated for $[\mathrm{M}+\mathrm{H}]=262.0429$, observed $[\mathrm{M}+\mathrm{H}]=262.0430$.

9-Acetyl-7-methoxy-9H-carbazole-3-carbaldehyde (2v). Prepared under constant potential conditions using $\mathbf{3 b}$ as catalyst. $\mathrm{CPE}$ carried out $1.9 \mathrm{~V}$ vs. $\mathrm{Ag}^{+} / \mathrm{Ag}$ with hfip/ $\mathrm{CH}_{2} \mathrm{Cl}_{2}(5 / 1)$ solvent mixture. Yield: $41 \mathrm{mg}$ (79\% yield). ${ }^{1} \mathrm{H}$ NMR $\left(\delta, 23{ }^{\circ} \mathrm{C}, 400 \mathrm{MHz}, \mathrm{CDCl}_{3}\right): 10.12$ (s, $\left.1 \mathrm{H}\right)$, 8.42 (dd, $J=1.7,0.5 \mathrm{~Hz}, 1 \mathrm{H}), 8.28(\mathrm{~d}, J=8.6 \mathrm{~Hz}, 1 \mathrm{H}), 7.96-7.91(\mathrm{~m}, 2 \mathrm{H}), 7.80(\mathrm{~d}, J=2.2 \mathrm{~Hz}$, $1 \mathrm{H}), 7.05(\mathrm{dd}, J=8.6,2.2 \mathrm{~Hz}, 1 \mathrm{H}), 3.94(\mathrm{~s}, 3 \mathrm{H}), 2.91(\mathrm{~s}, 3 \mathrm{H}) \cdot{ }^{13} \mathrm{C} \mathrm{NMR}\left(\delta, 23{ }^{\circ} \mathrm{C}, 100 \mathrm{MHz}, \mathrm{CDCl}_{3}\right):$ 191.6, 170.2, 160.4, 142.3, 140.7, 132.3, 128.0, 127.2, 120.9, 120.5, 119.0, 116.2, 111.7, 102.0, 55.9, 27.8. HRMS-ESI: calculated for $[\mathrm{M}+\mathrm{H}]=268.0968$, observed $[\mathrm{M}+\mathrm{H}]=268.0962$. 


\section{B.3 Intermolecular C-H Amination Electrocatalysis}

\section{General Procedure for Intermolecular Amination Reactions}

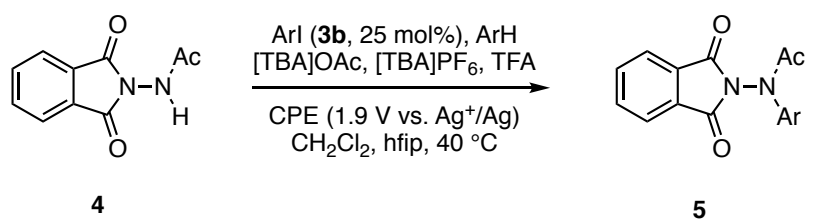

A 10-mL glass vial was charged with $N$-(1,3-dioxoisoindolin-2-yl)acetamide $(4,41.0 \mathrm{mg}$, $0.201 \mathrm{mmol}, 1.00$ equiv.), 2,2'-diiodo-4,4',6,6'-tetramethyl-1,1'-biphenyl (3b, $23.0 \mathrm{mg}$, $0.0495 \mathrm{mmol}, 24.6 \mathrm{~mol} \%$ ), arene ( $2.01 \mathrm{mmol}, 10.0$ equiv.), trifluoroacetic acid (TFA, $75.0 \mu \mathrm{L}$, 5.00 equiv.), tetrabutylammonium acetate (121 mg, $0.401 \mathrm{mmol}, 1.99$ equiv.), tetrabutylammonium hexafluorophosphate (390 mg, $1.01 \mathrm{mmol}, 5.02$ equiv.), hfip ( $4.2 \mathrm{~mL}$ ), and $\mathrm{CH}_{2} \mathrm{Cl}_{2}(0.8 \mathrm{~mL})$ and was fitted with glassy carbon anode, platinum cathode and $\mathrm{Ag}^{+} / \mathrm{Ag}$ reference electrode. A constant potential of $2.0 \mathrm{~V} \mathrm{vs}$. $\mathrm{Ag}^{+} / \mathrm{Ag}$ was applied to the reaction mixture and stirred at $40{ }^{\circ} \mathrm{C}$ until $\sim 80 \mathrm{C}$ charge $(\sim 4.15 \mathrm{~F} / \mathrm{mol})$ is passed. The solvent was then concentrated under reduced pressure. The obtained residue was purified by $\mathrm{SiO}_{2}$ gel chromatography (eluent 80:20 hexanes:ethyl acetate) to afford the products listed below.

$\mathrm{N}$-phenyl-N-(1,3-dioxoisoindolin-2-yl)acetamide (5a). Prepared from benzene at $1.8 \mathrm{~V}$ vs $\mathrm{Ag}^{+} / \mathrm{Ag}$. Yield: $40 \mathrm{mg}$ (71\% yield). With 5 equiv. of $\mathrm{PhH} 32 \%$ yield was obtained. ${ }^{1} \mathrm{H}$ NMR $(\delta$, $23{ }^{\circ} \mathrm{C}, 400 \mathrm{MHz}, \mathrm{CDCl}_{3}$ ): 7.89 (dd, $J=5.5,3.1 \mathrm{~Hz}, 2 \mathrm{H}$ ), $7.76(\mathrm{dd}, J=5.5,3.1 \mathrm{~Hz}, 2 \mathrm{H}$ ), 7.70-7.68 $(\mathrm{m}, 2 \mathrm{H}), 7.45(\mathrm{~d}, J=7.5 \mathrm{~Hz}, 3 \mathrm{H}), 2.11(\mathrm{~s}, 3 \mathrm{H}) .^{33}$

$\mathrm{N}$-(4-fluorophenyl)-N-(1,3-dioxoisoindolin-2-yl)acetamide (5b). Prepared from fluorobenzene. Yield: $21 \mathrm{mg}$ (35\% yield). ${ }^{1} \mathrm{H}$ NMR $\left(\delta, 23{ }^{\circ} \mathrm{C}, 400 \mathrm{MHz}, \mathrm{CDCl}_{3}\right): 7.89$ (dd, $J=$ 5.5, $3.0 \mathrm{~Hz}, 2 \mathrm{H}$ ), $7.77(\mathrm{dd}, J=5.5,3.1 \mathrm{~Hz}, 2 \mathrm{H}), 7.70(\mathrm{dd}, J=9.0,4.9 \mathrm{~Hz}, 2 \mathrm{H}), 7.14(\mathrm{dd}, J=9.0$, $8.2 \mathrm{~Hz}, 2 \mathrm{H}), 2.10(\mathrm{~s}, 3 \mathrm{H})$. The obtained spectral data are in good agreement with those reported in literature. ${ }^{34}$

$\mathrm{N}$-(4-chlorophenyl)-N-(1,3-dioxoisoindolin-2-yl)acetamide (5c). Prepared from chlorobenzene. Yield: $47 \mathrm{mg}$ (74\% yield). ${ }^{1} \mathrm{H}$ NMR $\left(\delta, 23{ }^{\circ} \mathrm{C}, 400 \mathrm{MHz}, \mathrm{CDCl}_{3}\right): 7.90$ (dd, $J=$ $5.5,3.1 \mathrm{~Hz}, 2 \mathrm{H}), 7.78(\mathrm{dd}, J=5.5,3.1 \mathrm{~Hz}, 2 \mathrm{H}), 7.63(\mathrm{~d}, J=8.7 \mathrm{~Hz}, 2 \mathrm{H}), 7.43(\mathrm{~d}, J=8.7 \mathrm{~Hz}, 2 \mathrm{H})$, $2.11(\mathrm{~s}, 3 \mathrm{H})$. The obtained spectral data are in good agreement with those reported in literature. 33

$\mathrm{N}$-(4-bromophenyl)-N-(1,3-dioxoisoindolin-2-yl)acetamide (5d). Prepared from bromobenzene. Yield: $59 \mathrm{mg}$ (82\% yield). ${ }^{1} \mathrm{H}$ NMR $\left(\delta, 23{ }^{\circ} \mathrm{C}, 400 \mathrm{MHz}, \mathrm{CDCl}_{3}\right): 7.89$ (dd, $J=$ $5.4,3.1 \mathrm{~Hz}, 2 \mathrm{H}), 7.78(\mathrm{dd}, J=5.5,3.1 \mathrm{~Hz}, 2 \mathrm{H}), 7.58(\mathrm{~d}, J=3.6 \mathrm{~Hz}, 4 \mathrm{H}), 2.11(\mathrm{~s}, 3 \mathrm{H})$. The obtained spectral data are in good agreement with those reported in literature. ${ }^{33}$

$\mathrm{N}$-(4-iodophenyl)-N-(1,3-dioxoisoindolin-2-yl)acetamide (5e). Prepared from iodobenzene. Yield: $58 \mathrm{mg}\left(72 \%\right.$ yield). ${ }^{1} \mathrm{H}$ NMR $\left(\delta, 23{ }^{\circ} \mathrm{C}, 400 \mathrm{MHz}, \mathrm{CDCl}_{3}\right): 7.89(\mathrm{dd}, J=5.5,3.1 \mathrm{~Hz}, 2 \mathrm{H})$, 7.80-7.76 (m, 4H), $7.42(\mathrm{~d}, J=8.5 \mathrm{~Hz}, 2 \mathrm{H}), 2.11(\mathrm{~s}, 3 \mathrm{H})$. The obtained spectral data are in good agreement with those reported in literature. ${ }^{34}$

$\mathrm{N}$-(3,4-diiodophenyl)-N-(1,3-dioxoisoindolin-2-yl)acetamideacetamide (5f). Prepared from 1,2-diiodobenzene (5 equiv.). Yield: $64 \mathrm{mg}\left(60 \%\right.$ yield). ${ }^{1} \mathrm{H} \operatorname{NMR}\left(\delta, 23{ }^{\circ} \mathrm{C}, 400 \mathrm{MHz}, \mathrm{CDCl}_{3}\right)$ : 
$8.16(\mathrm{~d}, J=2.1 \mathrm{~Hz}, 1 \mathrm{H}), 7.96-7.77(\mathrm{~m}, 5 \mathrm{H}), 7.37(\mathrm{dd}, J=8.4,2.1 \mathrm{~Hz}, 1 \mathrm{H}), 2.12(\mathrm{~s}, 3 \mathrm{H}) .{ }^{13} \mathrm{C}$ NMR $\left(\delta, 23{ }^{\circ} \mathrm{C}, 100 \mathrm{MHz}, \mathrm{CDCl}_{3}\right):$ 167.8, 164.9, 141.0, 140.5, 139.4, 135.0, 130.0, 129.7, 124.3, 109.9, 108.9, 21.8. HRMS-ESI: calculated for $[\mathrm{M}+\mathrm{H}]=532.8854$, observed $[\mathrm{M}+\mathrm{H}]=532.8844$.

$\mathrm{N}-(1,3-$ dioxoisoindolin-2-yl)-N-(3-iodo-4-methylphenyl)acetamide (5g). Prepared from 2iodotoluene (5 equiv.). Yield: $62 \mathrm{mg}$ (74\% yield) with 1:10 mixture of $\mathrm{O}^{-}(\mathrm{N}-(1,3-$ dioxoisoindolin-2-yl)- $N$-(3-iodo-2-methylphenyl)acetamide $)$ and $p$-isomer $(N-(1,3-$ dioxoisoindolin-2-yl)-N-(3-iodo-4-methylphenyl)acetamide). ${ }^{1} \mathrm{H}$ NMR $\left(\delta, 23{ }^{\circ} \mathrm{C}, 500 \mathrm{MHz}\right.$, $\mathrm{CDCl}_{3}, p$-isomer): $7.90-7.88(\mathrm{~m}, 3 \mathrm{H}), 7.77(\mathrm{dd}, J=5.5,3.1 \mathrm{~Hz}, 2 \mathrm{H}), 7.54(\mathrm{~s}, 1 \mathrm{H}), 7.21(\mathrm{dd}, J=$ 8.3, $2.4 \mathrm{~Hz}, 1 \mathrm{H}), 2.45(\mathrm{~s}, 3 \mathrm{H}), 2.11(\mathrm{~s}, 3 \mathrm{H}) .{ }^{13} \mathrm{C} \mathrm{NMR}\left(\delta, 23{ }^{\circ} \mathrm{C}, 125 \mathrm{MHz}, \mathrm{CDCl}_{3}, p\right.$-isomer $)$ : 168.2, 165.0, 143.9, 140.8, 140.4, 134.9, 130.1, 129.8, 127.7, 124.5, 124.2, 102.5, 28.3, 21.8. HRMS-ESI: calculated for $[\mathrm{M}+\mathrm{H}]=421.0044$, observed $[\mathrm{M}+\mathrm{H}]=421.0044$.

N-(2,4-dichlorophenyl)-N-(1,3-dioxoisoindolin-2-yl)acetamide (5h). Prepared from 1,3dichlorobenzene. Yield: $31 \mathrm{mg}\left(44 \%\right.$ yield). ${ }^{1} \mathrm{H}$ NMR $\left(\delta, 23{ }^{\circ} \mathrm{C}, 400 \mathrm{MHz}, \mathrm{CDCl}_{3}\right): 7.90(\mathrm{dd}, J=$ 5.4, 3.2 Hz, 2H), $7.84(\mathrm{~d}, J=8.6 \mathrm{~Hz}, 1 \mathrm{H}), 7.79(\mathrm{dd}, J=5.5,3.1 \mathrm{~Hz}, 2 \mathrm{H}), 7.56(\mathrm{~d}, J=2.3 \mathrm{~Hz}, 1 \mathrm{H})$, $7.34(\mathrm{dd}, J=8.6,2.4 \mathrm{~Hz}, 1 \mathrm{H}), 2.13(\mathrm{~s}, 3 \mathrm{H}) .{ }^{13} \mathrm{C} \mathrm{NMR}\left(\delta, 23{ }^{\circ} \mathrm{C}, 100 \mathrm{MHz}, \mathrm{CDCl}_{3}\right): 168.1,136.8$, 136.4, 134.8, 131.9, 131.0, 129.5, 128.8, 20.9. HRMS-ESI: calculated for $[\mathrm{M}+\mathrm{H}]=349.0141$, observed $[\mathrm{M}+\mathrm{H}]=349.0134$.

Ethyl 2-(N-(1,3-dioxoisoindolin-2-yl)acetamido)-5-iodobenzoate (5i). Prepared from ethyl 3iodobenzenoate (5 equiv.). Yield: $53 \mathrm{mg}$ (56\% yield). ${ }^{1} \mathrm{H}$ NMR $\left(\delta, 23{ }^{\circ} \mathrm{C}, 400 \mathrm{MHz}, \mathrm{CDCl}_{3}\right)$ : $8.20(\mathrm{~d}, J=2.1 \mathrm{~Hz}, 1 \mathrm{H}), 7.90(\mathrm{dd}, J=8.4,2.1 \mathrm{~Hz}, 1 \mathrm{H}), 7.85(\mathrm{~d}, J=8.5 \mathrm{~Hz}, 2 \mathrm{H}), 7.75(\mathrm{dd}, J=5.5$, $3.1 \mathrm{~Hz}, 2 \mathrm{H}), 7.55(\mathrm{~d}, J=8.4 \mathrm{~Hz}, 1 \mathrm{H}), 4.53(\mathrm{q}, J=7.2 \mathrm{~Hz}, 2 \mathrm{H}), 2.16(\mathrm{~s}, 3 \mathrm{H}), 1.41(\mathrm{t}, J=7.2 \mathrm{~Hz}$, $3 \mathrm{H}) .{ }^{13} \mathrm{C}$ NMR $\left(\delta, 23{ }^{\circ} \mathrm{C}, 100 \mathrm{MHz}, \mathrm{CDCl}_{3}\right): 169.0,164.97,164.91,142.3,140.2,138.6,134.9$, 134.4, 132.4, 130.0, 124.2, 95.7, 62.8, 21.9, 14.1. HRMS-ESI: calculated for $[\mathrm{M}+\mathrm{H}]=479.0098$, observed $[\mathrm{M}+\mathrm{H}]=479.0101$.

\section{B.3.1 Derivatization of Intermolecular C-H Amination Products}

\section{Synthesis of Acetanilide (6)}

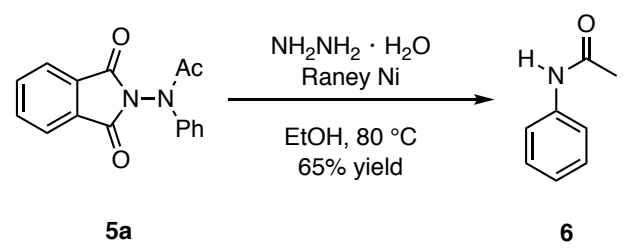

A $10-\mathrm{mL}$ round-bottom flask was charged with $N$-phenyl- $N$-(1,3-dioxoisoindolin-2yl)acetamide (5a, $50.1 \mathrm{mg}, 0.178 \mathrm{mmol}, 1.00$ equiv.), $80 \% \mathrm{~N}_{2} \mathrm{H}_{4} \cdot \mathrm{H}_{2} \mathrm{O}(30.0 \mu \mathrm{L}, 0.428 \mathrm{mmol}$, 2.88 equiv.), Raney $\mathrm{Ni}(150 \mathrm{mg})$, and ethanol $(5.0 \mathrm{~mL})$ and was fitted with a reflux condenser. The reaction mixture was refluxed for $2 \mathrm{~h}$ and then cooled to $23^{\circ} \mathrm{C}$. Benzene $(\sim 5 \mathrm{~mL})$ was added to the reaction mixture and the insoluble residue was removed by filtration. The filtrate was concentrated under reduced and the obtained residue was purified by $\mathrm{SiO}_{2}$ gel chromatography (eluent 80:20 hexanes:ethyl acetate) to afford the title compound as a white solid (16 mg, 65\% yield). ${ }^{1} \mathrm{H}$ NMR $\left(\delta, 23{ }^{\circ} \mathrm{C}, 400 \mathrm{MHz}, \mathrm{CDCl}_{3}\right): 7.50-7.48(\mathrm{~m}, 2 \mathrm{H}), 7.35-7.32$ $(\mathrm{m}, 3 \mathrm{H}), 7.13-7.09(\mathrm{~m}, 1 \mathrm{H}), 2.18(\mathrm{~s}, 3 \mathrm{H})$. The obtained spectral data are in good agreement with those reported in literature. ${ }^{35}$ 


\section{Synthesis of $N$-phenylacetohydrazide (7)}

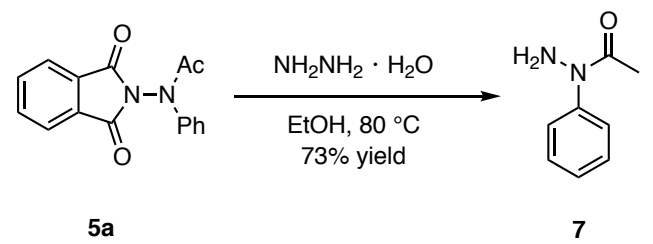

A 10-mL round-bottom flask was charged with $N$-phenyl- $N$-(1,3-dioxoisoindolin-2yl)acetamide (5a, $48.0 \mathrm{mg}, 0.171 \mathrm{mmol}, 1.00$ equiv.), $80 \% \mathrm{~N}_{2} \mathrm{H}_{4} \cdot \mathrm{H}_{2} \mathrm{O}(25.0 \mu \mathrm{L}, 0.428 \mathrm{mmol}$, 2.50 equiv.), and ethanol $(5.0 \mathrm{~mL})$ and was fitted with a reflux condenser. The reaction mixture was refluxed for $2 \mathrm{~h}$ and then cooled to $23^{\circ} \mathrm{C}$. Benzene $(\sim 5 \mathrm{~mL})$ was added to the reaction mixture and the insoluble residue was removed by filtration. The filtrate was then concentrated under reduced pressure. The obtained residue was purified by $\mathrm{SiO}_{2}$ gel chromatography (eluent 80:20 hexanes:ethyl acetate) to afford the title compound as a white solid (18 mg, 73\% yield). ${ }^{1} \mathrm{H}$ NMR $\left(\delta, 23{ }^{\circ} \mathrm{C}, 400 \mathrm{MHz}, \mathrm{CDCl}_{3}\right): 7.41(\mathrm{t}, J=7.5 \mathrm{~Hz}, 2 \mathrm{H}), 7.33-$ $7.28(\mathrm{~m}, 3 \mathrm{H}), 4.83(\mathrm{~s}, 2 \mathrm{H}), 2.00(\mathrm{~s}, 3 \mathrm{H})$. The obtained spectral data are in good agreement with those reported in literature. ${ }^{36}$ 


\section{Supporting Data}

Table S1. Onset potentials for oxidation of various substituted aryl iodides (5.0 mM) in 0.2 $\mathrm{M}$ [TBA] $\mathrm{PF}_{6}$ solution of hfip with glassy carbon working electrode, Pt counter electrode and $\mathrm{AgNO}_{3} / \mathrm{Ag}$ reference electrode. Scan rate $=0.10 \mathrm{~V} / \mathrm{s}$

\begin{tabular}{ccc}
\hline Entry & ArI & Onset potential $/ \mathbf{~ V ~ v s . ~} \mathbf{A g}^{+} / \mathbf{A g}$ \\
\hline 1 & 4-iodoanisole & 1.4 \\
2 & 2,4-dimethoxyiodobenzene & 1.41 \\
3 & 2,6-dimethoxyiodobenzene & 1.41 \\
4 & 4-iodotoluene & 1.65 \\
5 & 2,2'-diiodo-4,4',6,6'-tetramethyl-1,1'-biphenyl & $1.68,1.83$ \\
6 & iodobenzene & 1.74 \\
7 & 4-fluoroiodobenzene & 1.79 \\
8 & 2-iodobenzoic acid & 1.90 \\
9 & 4-trifluoromethyliodobenzene & 2.00 \\
\hline
\end{tabular}


Table S2. Effect of supporting electrolyte concentration on intramolecular $\mathrm{C}-\mathrm{H}$ amination yield. Reaction conditions: $0.20 \mathrm{mmol}$ of substrate, $0.05 \mathrm{mmol}$ of catalyst, $5.0 \mathrm{~mL}$ of solvent, and $\mathrm{AgNO}_{3} / \mathrm{Ag}$ reference electrode.

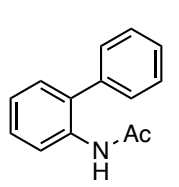

1a

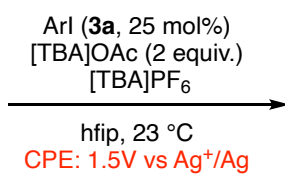

$$
\text { CPE: } 1.5 \mathrm{~V} \text { vs } \mathrm{Ag}^{+} / \mathrm{Ag}
$$

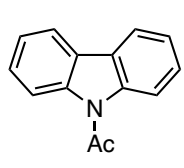

$2 a$

\begin{tabular}{ccc}
\hline Entry & [TBA]PF 6 Loading & Yield \\
\hline 1 & $0.05 \mathrm{M}$ & $60 \%$ \\
2 & $0.10 \mathrm{M}$ & $70 \%$ \\
3 & $0.15 \mathrm{M}$ & $68 \%$ \\
4 & $0.20 \mathrm{M}$ & $78 \%$ \\
\hline
\end{tabular}


Table S3. Comparison of the electrolyte concentration used in this manuscript with those in other recent electrocatalytic methods.

\begin{tabular}{|c|c|c|}
\hline Entry & Reference & $\begin{array}{l}\text { Electrolyte } \\
\text { Conc. (M) }\end{array}$ \\
\hline 1 & J. Am. Chem. Soc. 2019, 141, 2825-2831 & 0.1 \\
\hline 2 & ACS Catal. 2019, 9, 746-754 & 0.1 \\
\hline 3 & J. Am. Chem. Soc. 2018, 140, 12511-12520 & 0.1 \\
\hline 4 & J. Am. Chem. Soc. 2018, 140, 2438-2441 & 0.1 \\
\hline 5 & J. Am. Chem. Soc. 2018, 140, 2076-2079 & 0.1 \\
\hline 6 & J. Am. Chem. Soc. 2017, 139, 15548-15553 & 0.1 \\
\hline 7 & Science 2017, 357, 575-579 & 0.1 \\
\hline 8 & Chem. Eur. J. 2018, 24, 12274-12279 & 0.2 \\
\hline 9 & J. Am. Chem. Soc. 2017, 139, 7448-7451 & 0.05 \\
\hline 10 & Nature 2019, 573, 398. & 0.1 \\
\hline 11 & J. Am. Chem. Soc. 2019, 141, 6392-6402 & 0.2 \\
\hline 12 & Angew. Chem. Int. Ed. 2017, 56, 13088-13093 & 0.4 \\
\hline 13 & ACS Cent. Sci. 2019, 5, 1179-1186 & 0.5 \\
\hline 14 & Acc. Chem. Res. 2019, 52, 3432-3441 & 0.1 \\
\hline 15 & J. Am. Chem. Soc. 2019, 141, 14160-14167 & 0.1 \\
\hline 16 & J. Am. Chem. Soc. 2019, 141, 11115-11122 & 0.1 \\
\hline 17 & Angew. Chem. Int. Ed. 2018, 57, 10221-10225 & 0.1 \\
\hline 18 & J. Am. Chem. Soc. 2003, 125, 36-37. & 0.4 \\
\hline 19 & Angew. Chem., Int. Ed. 2010, 49, 129-133. & 0.1 \\
\hline 20 & Angew. Chem., Int. Ed. 2015, 54, 10555-10558. & 0.1 \\
\hline 21 & This work & 0.2 \\
\hline
\end{tabular}


Table S4. Effect of [TBA] OAc loading on intramolecular $\mathrm{CH}$ amination. Reaction conditions: $0.20 \mathrm{mmol}$ of substrate, $0.05 \mathrm{mmol}$ of catalyst, $5.0 \mathrm{~mL}$ of solvent, glassy carbon anode, $\mathrm{Pt}$ cathode, and $\mathrm{AgNO}_{3} / \mathrm{Ag}$ reference electrode.

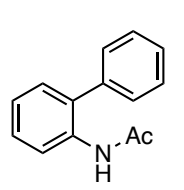

$1 a$

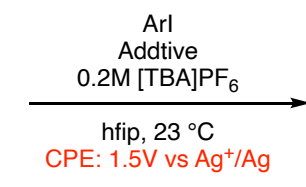

$$
\text { CPE: } 1.5 \mathrm{~V} \text { vs } \mathrm{Ag}^{+} / \mathrm{Ag}
$$
ArI Loading $25 \mathrm{~mol} \%$

$25 \mathrm{~mol} \%$ $25 \mathrm{~mol} \%$ $25 \mathrm{~mol} \%$ $25 \mathrm{~mol} \%$

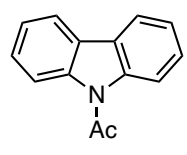

$2 a$

\begin{tabular}{ccccc}
\hline Entry & ArI & ArI Loading & Additive & Yield (\%) \\
\hline 1 & 4-iodoanisole & $25 \mathrm{~mol} \%$ & {$[\mathrm{TBA}]$ OAc (2 equiv.) } & 76 \\
2 & - & - & {$[\mathrm{TBA}]$ OAc (2 equiv.) } & trace \\
3 & 4-iodoanisole & $25 \mathrm{~mol} \%$ & - & 0 \\
4 & 4-iodoanisole & $25 \mathrm{~mol} \%$ & {$[\mathrm{TBA}]$ OAc (1 equiv.) } & 49 \\
5 & 4-iodoanisole & $25 \mathrm{~mol} \%$ & {$[\mathrm{TBA}]$ OAc (3 equiv.) } & 75 \\
6 & 4-iodoanisole & $25 \mathrm{~mol} \%$ & {$[\mathrm{TBA}] \mathrm{OAc}(5$ equiv.) } & 70 \\
\hline
\end{tabular}


Table S5. Optimization of intramolecular $\mathrm{C}-\mathrm{H}$ amination, varying solvent and temperature. Reaction conditions: $0.20 \mathrm{mmol}$ of substrate, $0.05 \mathrm{mmol}$ of catalyst, $0.40 \mathrm{mmol}$ [TBA] OAc, 0.2 $\mathrm{M}[\mathrm{TBA}] \mathrm{PF}_{6}, 5.0 \mathrm{~mL}$ of solvent, glassy carbon anode, Pt cathode, and $\mathrm{AgNO}_{3} / \mathrm{Ag}$ reference electrode.

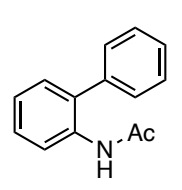

1a

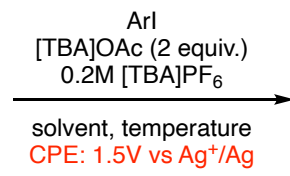

CPE: $1.5 \mathrm{~V}$ vs $\mathrm{Ag}^{+} / \mathrm{Ag}$

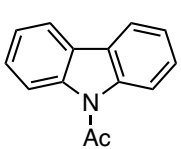

2a

\begin{tabular}{|c|c|c|c|c|c|}
\hline Entry & ArI & ArI Loading & Temperature & Solvent & Yield (\%) \\
\hline 1 & 4-iodoanisole & 1 equiv. & $23^{\circ} \mathrm{C}$ & hfip & 61 \\
\hline 2 & 4-iodoanisole & $25 \mathrm{~mol} \%$ & $23^{\circ} \mathrm{C}$ & hfip & 76 \\
\hline 3 & 4-iodoanisole & $20 \mathrm{~mol} \%$ & $23^{\circ} \mathrm{C}$ & hfip & 56 \\
\hline 4 & 4-iodoanisole & $15 \mathrm{~mol} \%$ & $23^{\circ} \mathrm{C}$ & hfip & 40 \\
\hline 5 & 4-iodotoluene & $25 \mathrm{~mol} \%$ & $23^{\circ} \mathrm{C}$ & hfip & 0 \\
\hline 6 & iodobenzene & $25 \mathrm{~mol} \%$ & $23^{\circ} \mathrm{C}$ & hfip & 0 \\
\hline 7 & 4-iodoanisole & $25 \mathrm{~mol} \%$ & $23^{\circ} \mathrm{C}$ & $\mathrm{MeCN}$ & 0 \\
\hline 8 & 4-iodoanisole & $25 \mathrm{~mol} \%$ & $23^{\circ} \mathrm{C}$ & $\mathrm{CH}_{2} \mathrm{Cl}_{2}$ & 0 \\
\hline 9 & 4-iodoanisole & $25 \mathrm{~mol} \%$ & $23^{\circ} \mathrm{C}$ & TFE & 28 \\
\hline 10 & 4-iodoanisole & $25 \mathrm{~mol} \%$ & $23^{\circ} \mathrm{C}$ & $\begin{array}{c}\text { hfip/MeCN } \\
(1 / 1)\end{array}$ & 0 \\
\hline 11 & 4-iodoanisole & $25 \mathrm{~mol} \%$ & $23^{\circ} \mathrm{C}$ & $\begin{array}{l}\text { hfip/MeCN } \\
(2 / 1)\end{array}$ & 0 \\
\hline 12 & 4-iodoanisole & $25 \mathrm{~mol} \%$ & $23^{\circ} \mathrm{C}$ & $\begin{array}{c}\text { hfip/MeCN } \\
(9 / 1)\end{array}$ & 45 \\
\hline 13 & 4-iodoanisole & $25 \mathrm{~mol} \%$ & $40^{\circ} \mathrm{C}$ & hfip & 37 \\
\hline
\end{tabular}


Table S6. Evaluation of the impact of the amine protecting group. Reaction conditions: 0.20 mmol of substrate, $0.05 \mathrm{mmol}$ of catalyst, $0.40 \mathrm{mmol}$ [TBA]OAc, $0.2 \mathrm{M}$ [TBA]PF $6,5.0 \mathrm{~mL}$ of hfip, glassy carbon anode, $\mathrm{Pt}$ cathode, and $\mathrm{AgNO}_{3} / \mathrm{Ag}$ reference electrode.

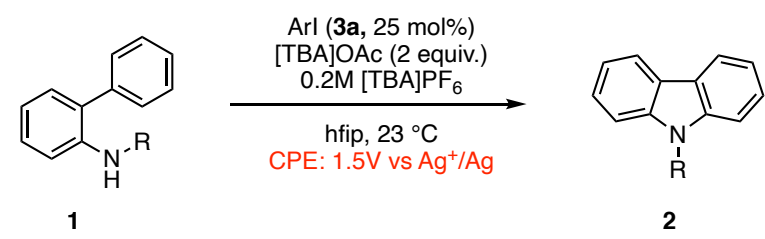

\begin{tabular}{ccc}
\hline Entry & R & Yield (\%) \\
\hline 1 & $\mathrm{Ac}$ & 76 \\
2 & $\mathrm{Bz}$ & 56 \\
3 & $\mathrm{Ms}$ & 64 \\
4 & $\mathrm{Ts}$ & 44 \\
5 & $\mathrm{CF}_{3} \mathrm{COO}$ & 25 \\
\hline
\end{tabular}


Table S7. Effect of electrode materials on intramolecular $\mathrm{C}-\mathrm{H}$ amination. Reaction conditions: $0.20 \mathrm{mmol}$ of substrate, $0.05 \mathrm{mmol}$ of catalyst, $5.0 \mathrm{~mL}$ of solvent, and $\mathrm{AgNO}_{3} / \mathrm{Ag}$ reference electrode.

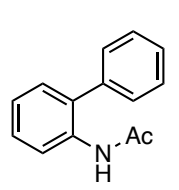

$1 a$

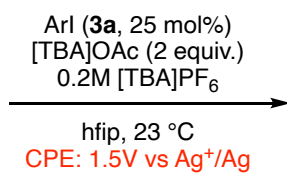
CPE: $1.5 \mathrm{~V}$ vs $\mathrm{Ag}^{+} / \mathrm{Ag}$

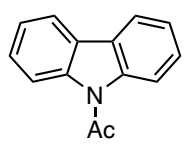

$2 a$

\begin{tabular}{cccc}
\hline Entry & Anode & Cathode & Yield (\%) \\
\hline 1 & Glassy Carbon & $\mathrm{Pt}$ & 76 \\
2 & $\mathrm{Pt}$ & $\mathrm{Pt}$ & 0 \\
3 & Graphite & $\mathrm{Pt}$ & $<10 \%$ \\
4 & Boron doped carbon (BDC) & $\mathrm{Pt}$ & $35 \%$ \\
\hline
\end{tabular}




\section{Electrolysis and headspace analysis of 4-iodotoluene in hfip}

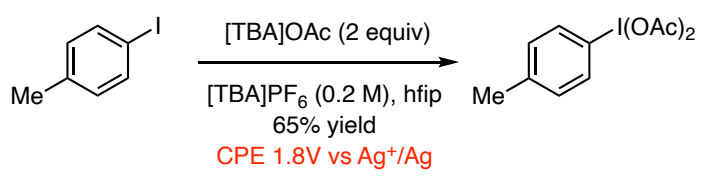

A 10 -mL glass vial was charged with 4-iodotoluene ( $43.1 \mathrm{mg}, 0.197 \mathrm{mmol}, 1.00$ equiv.), tetrabutylammonium acetate $(121 \mathrm{mg}, 0.401 \mathrm{mmol}, 2.03$ equiv.), tetrabutylammonium hexafluorophosphate $(390 \mathrm{mg}, 1.01 \mathrm{mmol}, 5.02$ equiv.), and hfip $(5.0 \mathrm{~mL})$. The reaction vessel was fitted with glassy carbon anode, platinum cathode, and $\mathrm{a}^{+} / \mathrm{Ag}$ reference electrode. A constant potential of $1.8 \mathrm{~V}$ vs. $\mathrm{Ag}^{+} / \mathrm{Ag}$ was applied to the reaction mixture and stirred at $23{ }^{\circ} \mathrm{C}$ until $\sim 80 \mathrm{C}$ charge $(\sim 4.15 \mathrm{~F} / \mathrm{mol})$ is passed. The headspace of the reaction mixture was analyzed by gas chromatography to determine the products of the cathodic half of the reaction. Data are collected in Figure S1.

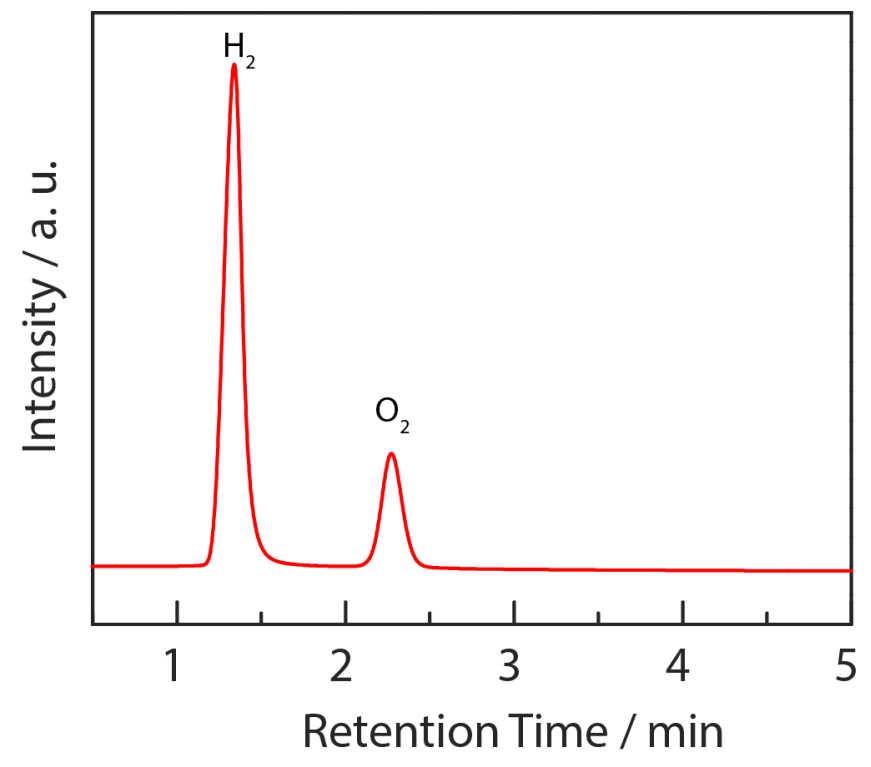

Figure S1. Gas chromatogram of the headspace of 4-iodotoluene oxidation in hfip indicating the evolution of $\mathrm{H}_{2}$ at the cathode. The oxygen $\left(\mathrm{O}_{2}\right)$ peak is from air; the reaction was set up under ambient conditions. 


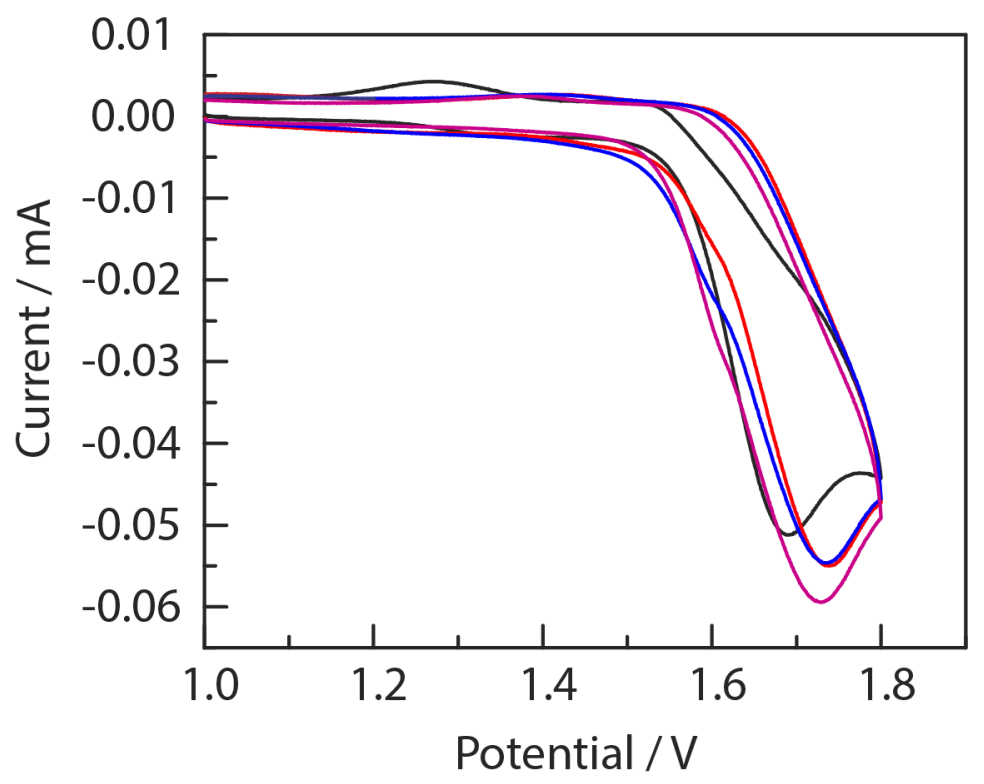

Figure S2. Cyclic voltammograms of the parent substrate 1a and halogenated substrates $\mathbf{1 b}$ 1d. Compound 1a $(-)$, compound $\mathbf{1 b}(-)$, compound $\mathbf{1 c}(-)$, and compound $\mathbf{1 d}(-)$. CV conditions: substrate $5 \mathrm{mM}, 0.2 \mathrm{M}[\mathrm{TBA}] \mathrm{PF}_{6}$ solution of hfip, glassy carbon working electrode, Pt counter electrode, and scan rate $=0.10 \mathrm{~V} / \mathrm{s}$.

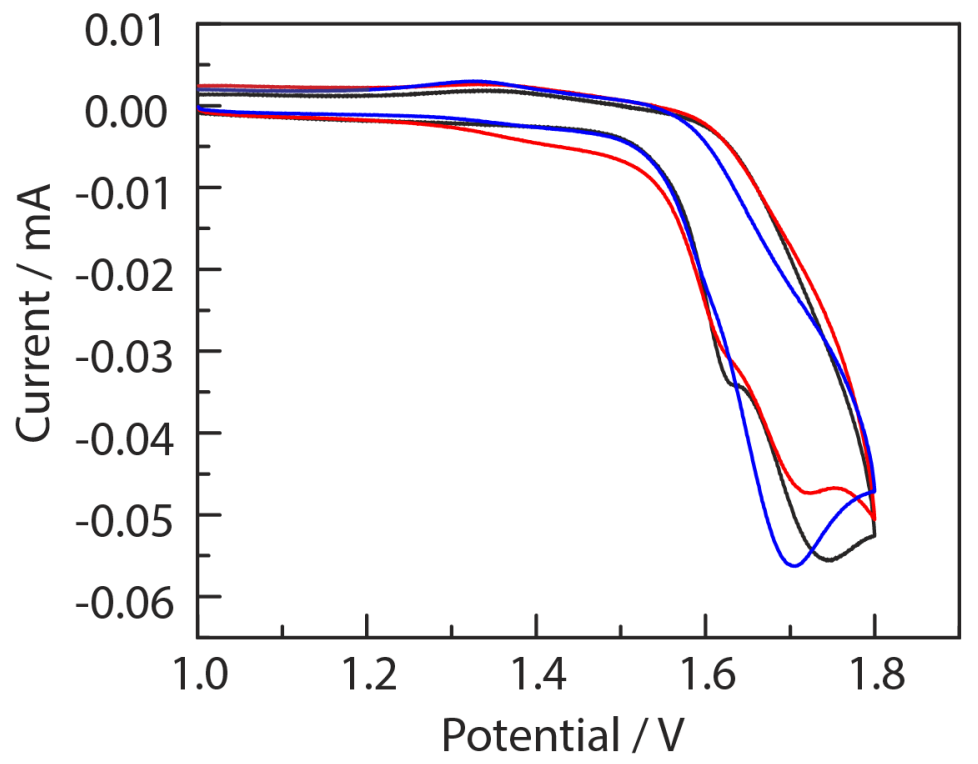

Figure S3. Cyclic voltammograms of halogenated substrates 1e-1g. Compound 1e $(-)$, compound $\mathbf{1 f}(-)$, and compound $\mathbf{1 g}(-)$. CV conditions: substrate $5 \mathrm{mM}, 0.2 \mathrm{M}[\mathrm{TBA}] \mathrm{PF}_{6}$ solution of hfip, glassy carbon working electrode, Pt counter electrode, and scan rate $=0.10$ $\mathrm{V} / \mathrm{s}$. 


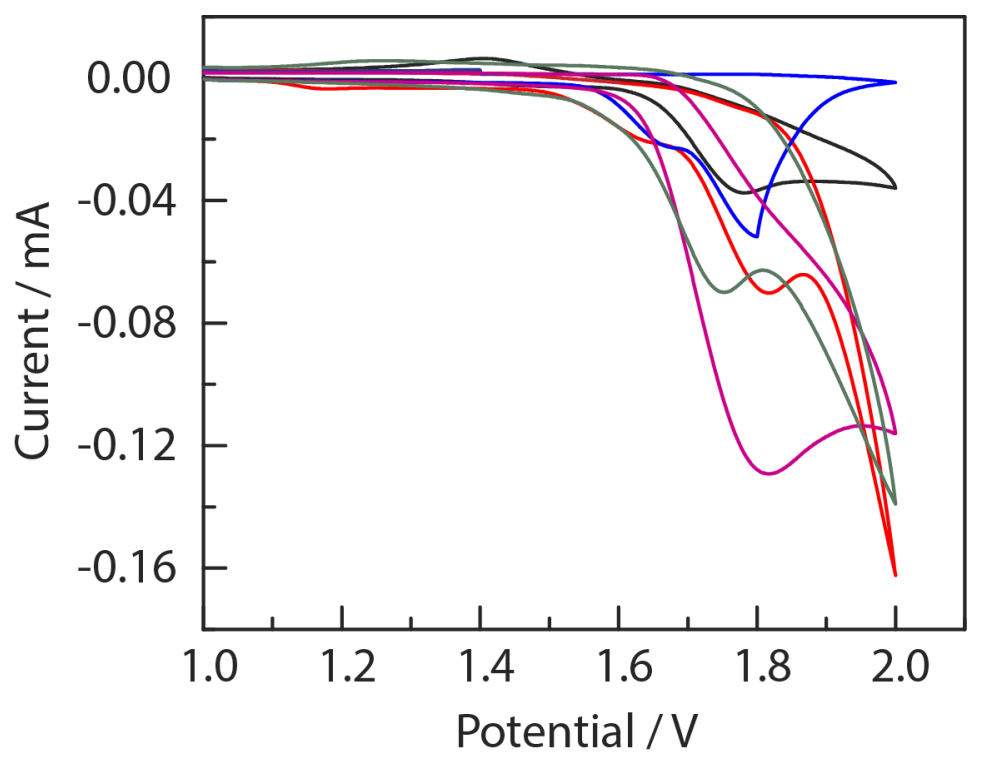

Figure S4. Cyclic voltammograms of halogenated substrates 1q-1u. Compound 1q (-), compound 1r (-), compound 1s (-), compound 1t (-), and compound 1u (-). CV conditions: substrate $5 \mathrm{mM}, 0.2 \mathrm{M}\left[\mathrm{TBA}^{\mathrm{P}} \mathrm{PF}_{6}\right.$ solution of hfip, glassy carbon working electrode, Pt counter electrode, and scan rate $=0.10 \mathrm{~V} / \mathrm{s}$.

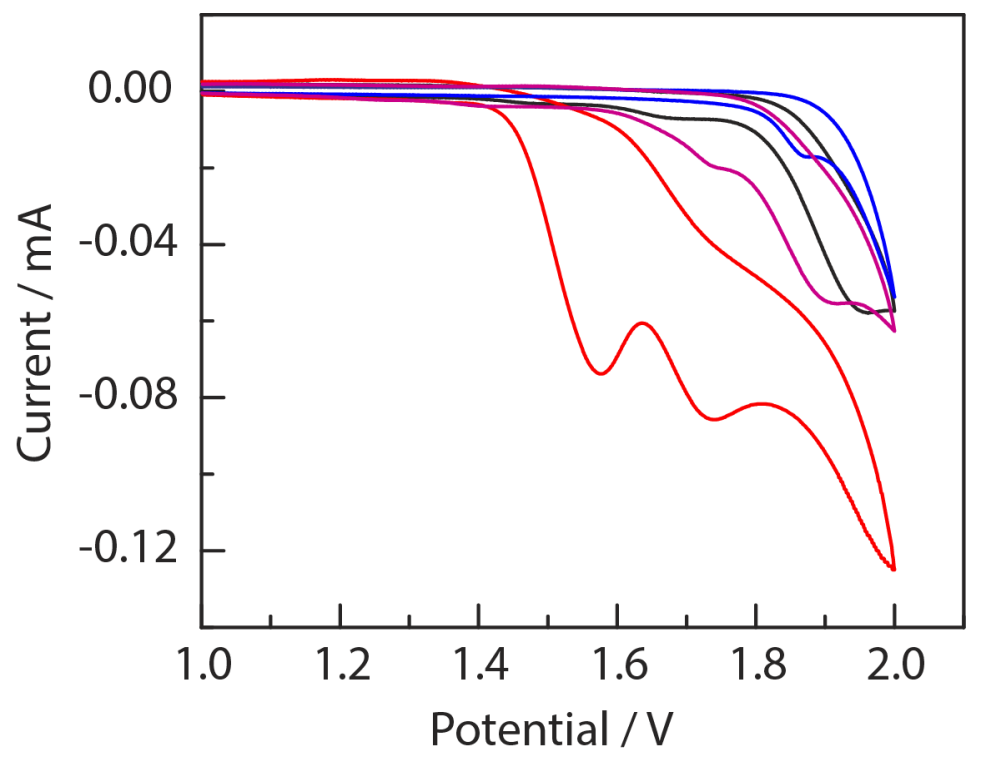

Figure S5. Cyclic voltammograms of electron poor substrates $\mathbf{1 h}-\mathbf{1 k}$. Compound $\mathbf{1 h}(-)$, compound $\mathbf{1 i}(-)$, compound $\mathbf{1 j}(-)$, and compound $\mathbf{1 k}(-)$. CV conditions: substrate $5 \mathrm{mM}$, $0.2 \mathrm{M}[\mathrm{TBA}] \mathrm{PF}_{6}$ solution of hfip, glassy carbon working electrode, Pt counter electrode, and scan rate $=0.10 \mathrm{~V} / \mathrm{s}$. 


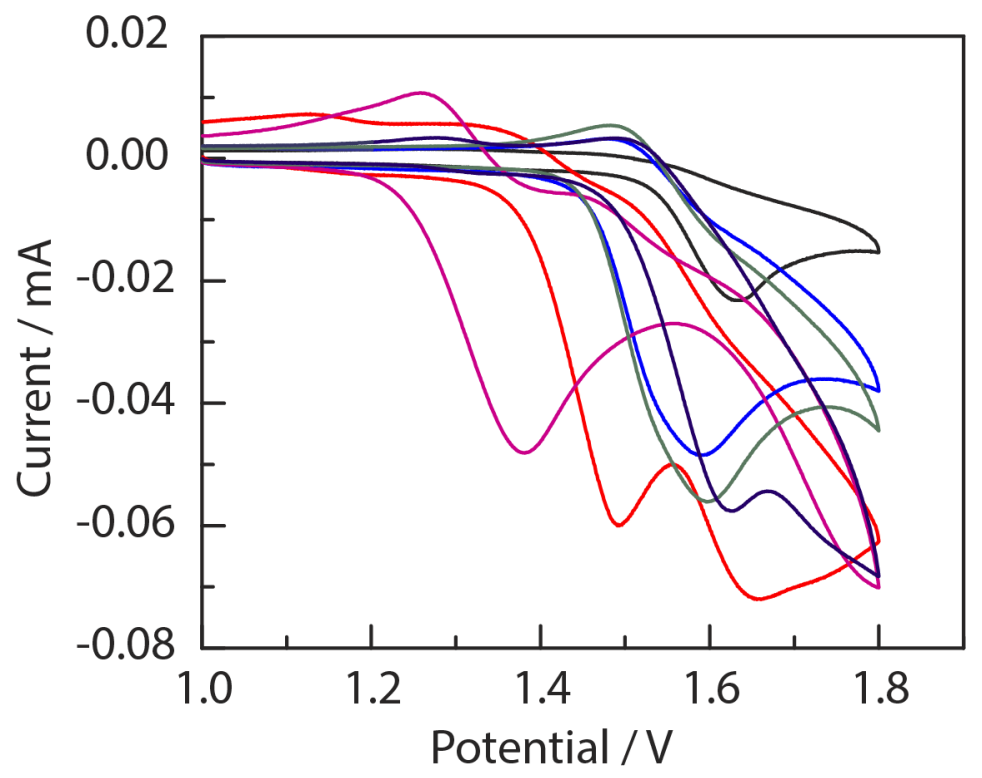

Figure S6. Cyclic voltammograms of electron rich substrates 1l-1p, 1v. Compound $11(-)$, compound 1m $(-)$, compound $1 \mathrm{n}(-)$, compound $1 \mathrm{o}(-)$, compound $1 \mathbf{p}(-)$, and compound $1 \mathbf{v}(-)$. CV conditions: substrate $5 \mathrm{mM}, 0.2 \mathrm{M}[\mathrm{TBA}] \mathrm{PF}_{6}$ solution of hfip, glassy carbon working electrode, Pt counter electrode, and scan rate $=0.10 \mathrm{~V} / \mathrm{s}$. 
Table S8. Onset and peak potentials of biarylacetamide substrates. Data collected from Figures S2-S6. CV conditions: substrate $5 \mathrm{mM}, 0.2 \mathrm{M}$ [TBA] $\mathrm{PF}_{6}$ solution of hfip, glassy carbon working electrode, Pt counter electrode, $\mathrm{Ag}^{+} / \mathrm{Ag}$ reference electrode and scan rate $=0.10 \mathrm{~V} / \mathrm{s}$.

\begin{tabular}{|cccc}
\hline Entry & Substrate & $\begin{array}{c}\text { Onset Potential } \\
\left(\mathbf{V}^{\mathbf{2}} \mathbf{A g}^{+} / \mathbf{A g}\right)\end{array}$ & $\begin{array}{c}\text { Peak Potential } \\
\left(\mathbf{V ~ v s ~ A g}^{+} / \mathbf{A g}\right)\end{array}$ \\
\hline 1 & $\mathbf{1 a}$ & 1.50 & 1.69 \\
\hline 2 & $\mathbf{1 b}$ & 1.50 & 1.73 \\
\hline 3 & $\mathbf{1 c}$ & 1.50 & 1.73 \\
\hline 4 & $\mathbf{1 d}$ & 1.55 & 1.72 \\
\hline 5 & $\mathbf{1 e}$ & 1.50 & 1.74 \\
6 & $\mathbf{1 f}$ & 1.50 & 1.71 \\
\hline 7 & $\mathbf{1 g}$ & 1.54 & 1.70 \\
\hline 8 & $\mathbf{1 h}$ & 1.76 & 1.96 \\
\hline 9 & $\mathbf{1 i}$ & 1.44 & $1.58,1.75$ \\
\hline 10 & $\mathbf{1 j}$ & 1.83 & 1.92 \\
11 & $\mathbf{1 k}$ & 1.62 & 1.90 \\
\hline 12 & $\mathbf{1 l}$ & 1.50 & 1.62 \\
\hline 13 & $\mathbf{1 m}$ & 1.34 & 1.50 \\
\hline 14 & $\mathbf{1 n}$ & 1.43 & 1.58 \\
\hline 15 & $\mathbf{1 0}$ & 1.22 & 1.39 \\
\hline 16 & $\mathbf{1 p}$ & 1.43 & 1.60 \\
\hline 17 & $\mathbf{1 q}$ & 1.61 & 1.80 \\
\hline 18 & $\mathbf{1 r}$ & 1.56 & $1.65,1.82$ \\
\hline 19 & $\mathbf{1 s}$ & 1.50 & 1.68 \\
\hline 20 & $\mathbf{1 t}$ & 1.61 & 1.83 \\
\hline 21 & $\mathbf{1 u}$ & 1.57 & 1.76 \\
\hline 22 & $\mathbf{1 v}$ & 1.48 & 1.63 \\
\hline
\end{tabular}


Table S9. Background reactions without aryliodide present.
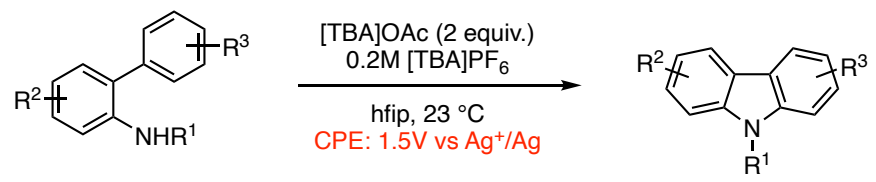

A 10-mL glass vial was charged with $N$-arylacetamide (1, $0.201 \mathrm{mmol}, 1.00$ equiv.), tetrabutylammonium acetate $(121 \mathrm{mg}, 0.401 \mathrm{mmol}, 1.99$ equiv.), tetrabutylammonium hexafluorophosphate (390 mg, $1.01 \mathrm{mmol}, 5.02$ equiv.), and hfip (5.0 mL) and was fitted with glassy carbon anode, platinum cathode, and $\mathrm{Ag}^{+} / \mathrm{Ag}$ reference electrode. A constant potential of $1.5 \mathrm{~V}$ vs. $\mathrm{Ag}^{+} / \mathrm{Ag}$ was applied to the reaction mixture with stirring at $23{ }^{\circ} \mathrm{C}$ until $\sim 80 \mathrm{C}$ charge $(\sim 4.15 \mathrm{~F} / \mathrm{mol})$ is passed if the current density was not too low. The solvent was removed under reduced pressure. Mesitylene was added directly to the crude reaction mixture as an internal standard and the yield was analyzed by ${ }^{1} \mathrm{H}$ NMR in $\mathrm{CDCl}_{3}$.

\begin{tabular}{ccccc} 
Entry & Substrate & $\begin{array}{c}\text { Yield With } \\
\text { ArI (\%) }\end{array}$ & $\begin{array}{c}\text { Yield Without } \\
\text { ArI (\%) }\end{array}$ & $\begin{array}{c}\text { Onset } \\
\text { Potential } \\
\text { (V vs Ag+/Ag) }\end{array}$ \\
\hline 1 & $\mathbf{1 a}, \mathrm{R}_{1}=\mathrm{R}_{2}=\mathrm{H}$ & 76 & $0^{\mathrm{a}}$ & 1.50 \\
\hline 2 & $\mathbf{1 b}, \mathrm{R}_{1}=\mathrm{Br}, \mathrm{R}_{2}=\mathrm{H}$ & 78 & $10^{\mathrm{a}}$ & 1.50 \\
\hline 3 & $\mathbf{1 c}, \mathrm{R}_{1}=\mathrm{Cl}, \mathrm{R}_{2}=\mathrm{H}$ & 80 & $15^{\mathrm{a}}$ & 1.50 \\
\hline 4 & $\mathbf{1 g}, \mathrm{R}_{1}=\mathrm{H}, \mathrm{R}_{2}=\mathrm{F}$ & 78 & $5^{\mathrm{a}}$ & 1.54 \\
\hline 5 & $\mathbf{1 j}, \mathrm{R}_{1}=\mathrm{NO}_{2}, \mathrm{R}_{2}=\mathrm{H}$ & 43 & $0^{\mathrm{a}}$ & 1.83 \\
\hline 6 & $\mathbf{1 k}, \mathrm{R}_{1}=\mathrm{COOM}, \mathrm{R}_{2}=\mathrm{H}$ & 71 & $0^{\mathrm{a}}$ & 1.62 \\
\hline 7 & $\mathbf{1 1}, \mathrm{R}_{1}=\mathrm{H}, \mathrm{R}_{2}=\mathrm{t} \mathrm{Bu}$ & 65 & $0^{\mathrm{a}}$ & 1.50 \\
\hline 8 & $\mathbf{1} \mathbf{m}, \mathrm{R}_{1}=\mathrm{H}, \mathrm{R}_{2}=\mathrm{OMe}$ & 44 & $0^{\mathrm{b}}$ & 1.34 \\
\hline 9 & $\mathbf{1 0}, \mathrm{R}_{1}=\mathrm{OMe}, \mathrm{R}_{2}=\mathrm{H}$ & 10 & $0^{\mathrm{b}}$ & 1.22 \\
\hline 11 & $\mathbf{1 p}, \mathrm{R}_{1}=\mathrm{t} \mathrm{Bu}, \mathrm{R}_{2}=\mathrm{H}$ & 65 & 65 & 1.43 \\
\hline 12 & $\mathbf{1 v}, \mathrm{R}_{1}=\mathrm{CHO}, \mathrm{R}_{2}=\mathrm{OMe}$ & 79 & 33 & 1.48 \\
\hline
\end{tabular}

${ }^{a}$ current density was too low. ${ }^{\mathrm{b}}$ decomposition of substrate was observed. 


\section{Robustness Analysis for the Intramolecular C-H Amination}

Hypervalent iodine catalyzed C-N bond forming chemistry is most often accomplished with peracid terminal oxidants. $2,27,34$ While recently developed aerobic hypervalent iodine chemistry proceeds through a distinct one-electron mechanism, the autoxidation chemistry used to couple $\mathrm{O}_{2}$ reduction to aryl iodide oxidation, produces a significant steady state concentration of peracid. For this reason, the developed aerobic oxidation conditions display substrate scope limitations similar to those displayed by peracid conditions when assayed by robustness analysis. ${ }^{37,38} \mathrm{We}$ were interested in evaluating the robustness of the developed hypervalent iodine electrocatalysis to evaluate if a broader functional group tolerance may be achieved by avoiding the use or evolution of peracids. Figure 4 displays both the impact of a variety of small-molecule additives on the yield of intramolecular C$\mathrm{H} / \mathrm{N}-\mathrm{H}$ coupling as well as the amount of recovered additive following the electrochemical reaction. The efficiency of electrocatalytic $\mathrm{C}-\mathrm{N}$ coupling is superior to aerobic conditions for all additives and similar to that of peracetic acid. The electrochemical conditions display higher additive recovery, in particular when challenged against oxidatively labile functional groups, such as alkynes and olefins ( $96 \%$ and $92 \%$, respectively).

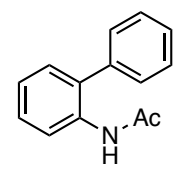

1a

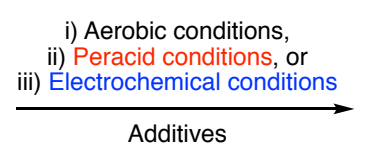

Additives

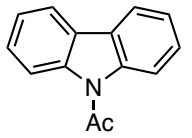

$2 a$

Aerobic conditions A 20 -mL scintillation vial was charged with $N$-([1,1'-biphenyl] -2 yl)acetamide (1a, $42.5 \mathrm{mg}, 0.201 \mathrm{mmol}, 1.00$ equiv.), 4-iodotoluene $(11.0 \mathrm{mg}, 0.0505 \mathrm{mmol}$, $25.0 \mathrm{~mol} \%$ ), $\mathrm{CoCl}_{2} \cdot 6 \mathrm{H}_{2} \mathrm{O}(0.5 \mathrm{mg}, 2.13 \mu \mathrm{mol}, 1.06 \mathrm{~mol} \%), 1,2$-dichloroethane (DCE, $1.0 \mathrm{~mL}$ ), and hfip $(0.50 \mathrm{~mL})$ and the reaction mixture was fitted with a rubber septum. The reaction vessel was purged with $\mathrm{O}_{2}$ for 2-3 min and acetaldehyde ( $120 \mu \mathrm{L}, 2.13 \mathrm{mmol}, 10.6$ equiv.) was added to the reaction vessel. Individual additives ( $0.201 \mathrm{mmol}, 1.00$ equiv.) were dissolved in 2:1 DCE:hfip $(0.50 \mathrm{~mL})$ and were added to the reaction vessel via syringe after $15 \mathrm{~min}$. The reaction mixture was stirred at $23{ }^{\circ} \mathrm{C}$ under $1 \mathrm{~atm} \mathrm{O}_{2}$, delivered by inflated balloon for $16 \mathrm{~h}$. Mesitylene was added as an internal standard to the crude reaction mixture and then an aliquot $(\sim 0.1 \mathrm{~mL})$ of the reaction mixture was taken into an NMR tube, diluted with $\mathrm{CDCl}_{3}(\sim 0.4 \mathrm{ml})$, and corresponding ${ }^{1} \mathrm{H}$ NMR was recorded. The yield and additive recovery were analyzed from the appropriate ${ }^{1} \mathrm{H}$ NMR. Data are collected in the Figure S7.

Peracid conditions A 20 -mL scintillation vial was charged with $N$-([1,1'-biphenyl $]-2$ yl)acetamide (1a, $32.1 \mathrm{mg}, 0.152 \mathrm{mmol}, 1.00$ equiv.), 2,2'-diiodo-4,4',6,6'-tetramethyl-1,1'biphenyl (3b, $7.07 \mathrm{mg}, 0.0151 \mathrm{mmol}, 10.0 \mathrm{~mol} \%$ ), dichloromethane $\left(\mathrm{CH}_{2} \mathrm{Cl}_{2}, 1.50 \mathrm{~mL}\right)$, hfip $(1.50 \mathrm{~mL})$, and appropriate additives $(0.151 \mathrm{mmol}, 1.00$ equiv.). Peracetic acid (39\% in acetic acid, $51.0 \mu \mathrm{L}, 0.301 \mathrm{mmol}, 1.99$ equiv.) was added to the reaction vessel, and the reaction mixture was stirred at $23{ }^{\circ} \mathrm{C}$ for $16 \mathrm{~h}$. Mesitylene was added as an internal standard to the crude reaction mixture and then an aliquot $(\sim 0.1 \mathrm{~mL})$ of the reaction mixture was taken into an NMR tube, diluted with $\mathrm{CDCl}_{3}(\sim 0.4 \mathrm{ml})$, and corresponding ${ }^{1} \mathrm{H}$ NMR was recorded. The 
yield and additive recovery were analyzed from the appropriate ${ }^{1} \mathrm{H}$ NMR. Data are collected in the Figure S7.

Electrochemical conditions. A 10 -mL glass vial was charged with $N$-([1,1'-biphenyl] -2 yl)acetamide (1a, $0.201 \mathrm{mmol}, 1.00$ equiv.), 4-iodoanisole $(12.0 \mathrm{mg}, 0.0512 \mathrm{mmol}, 25.3$ mol\%), appropriate additive ( $0.201 \mathrm{mmol}, 1.00$ equiv.), tetrabutylammonium acetate (121 $\mathrm{mg}, 0.401 \mathrm{mmol}, 1.99$ equiv.), tetrabutylammonium hexafluorophosphate (390 mg, 1.01 mmol, 5.02 equiv.), and hfip $(5.0 \mathrm{~mL})$. The reaction vessel was fitted with glassy carbon anode, platinum cathode, and $\mathrm{Ag}^{+} / \mathrm{Ag}$ reference electrode. A constant potential of $1.5 \mathrm{~V}$ vs. $\mathrm{Ag}^{+} / \mathrm{Ag}$ was applied to the reaction mixture, which was stirred at $23{ }^{\circ} \mathrm{C}$ until $\sim 80 \mathrm{C}$ charge ( $\sim 4.15 \mathrm{~F} / \mathrm{mol})$ was passed. Mesitylene was added as an internal standard to the crude reaction mixture and then an aliquot $(\sim 0.1 \mathrm{~mL})$ of the reaction mixture was taken into an NMR tube, diluted with $\mathrm{CDCl}_{3}(\sim 0.4 \mathrm{ml})$, and corresponding ${ }^{1} \mathrm{H}$ NMR was recorded. The yield and additive recovery were analyzed from the appropriate ${ }^{1} \mathrm{H}$ NMR. Data are collected in the Figure S7.

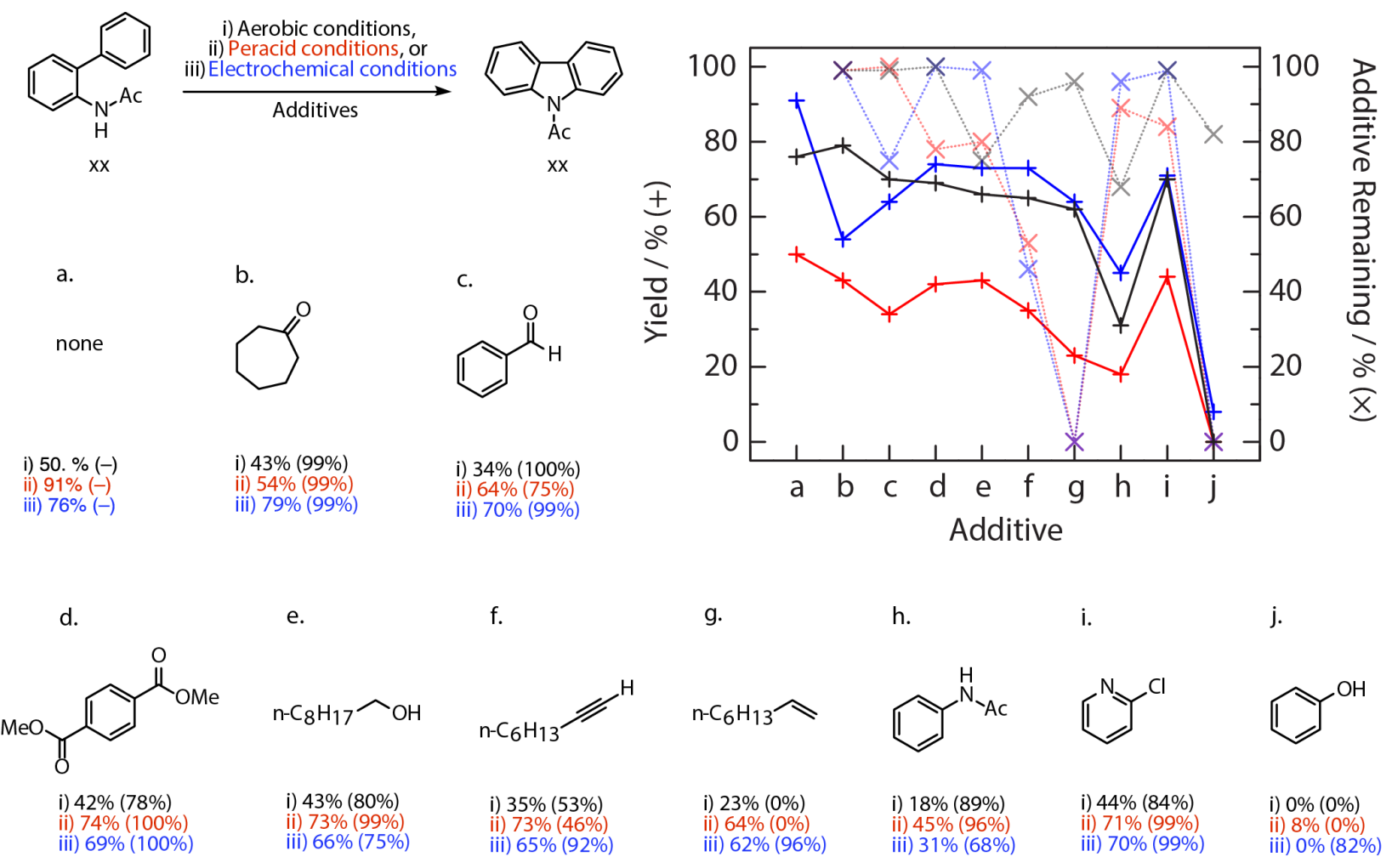

Figure S7. Summary of the results of robustness analysis. Reaction yield (solid lines) and additive recovery (dotted lines) for aerobic conditions $(-, \cdots)$, m-CPBA conditions $(-, \cdots)$, and electrochemical conditions $(-, \cdots)$. The functional group tolerance for electrochemical conditions ( - ) is superior than aerobic conditions ( - ) and comparable with peracid conditions (-). 
Table S10. Optimization of intermolecular $\mathrm{C}-\mathrm{H}$ amination. Reaction conditions: $0.20 \mathrm{mmol}$ of substrate, $0.05 \mathrm{mmol}$ of catalyst, $0.40 \mathrm{mmol}$ [TBA]OAc, $0.2 \mathrm{M}$ [TBA]PF, $5.0 \mathrm{~mL}$ of solvent $\left(\mathrm{CH}_{2} \mathrm{Cl}_{2} / \mathrm{hfip}: 5 / 1\right)$, glassy carbon anode, $\mathrm{Pt}$ cathode, and $\mathrm{AgNO}_{3} / \mathrm{Ag}$ reference electrode.

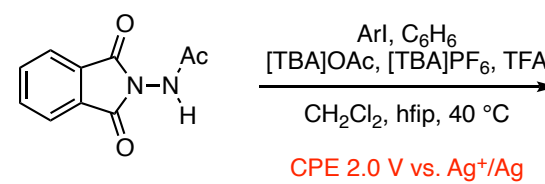

4

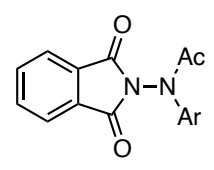

5

\begin{tabular}{cccc}
\hline Entry & ArI & ArI Loading & Yield (\%) \\
\hline 1 & 4-iodoanisole & 1 equiv. & 0 \\
2 & Iodobenzene & 1 equiv. & 0 \\
3 & 4-iodotoluene & 1 equiv. & 0 \\
4 & 1,2-diiodobenzene & 1 equiv. & 65 \\
5 & $2,2^{\prime}$ '-diiodo-4,4',6,6'-tetramethyl-1,1'-biphenyl & 1 equiv. & 81 \\
6 & $2,2^{\prime}$-diiodo-4,4',6,6'-tetramethyl-1,1'-biphenyl & 25 mol\% & 71 \\
7 & 2,2'-diiodo-4,4',6,6'-tetramethyl-1,1'-biphenyl & 20 mol\% & 59 \\
\hline
\end{tabular}




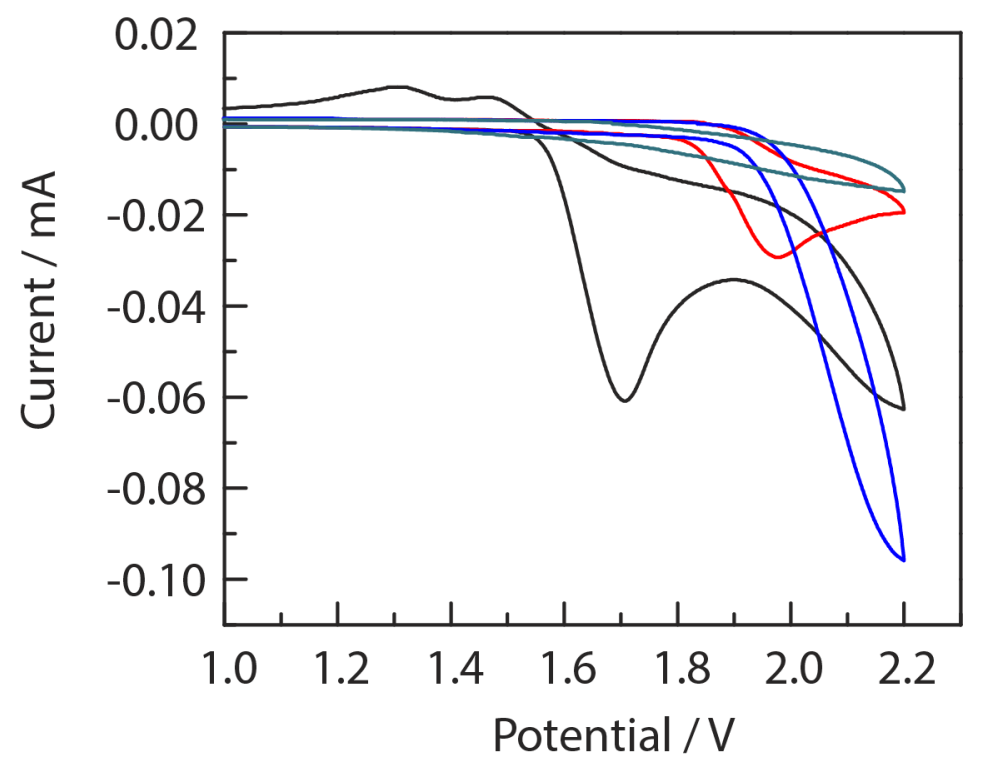

Figure S8. Cyclic voltammograms of various amines for intermolecular chemistryacetanilide (-), TrocNHOMe (-), TsNHOMe (-) and $N$-(1,3-dioxoisoindolin-2yl)acetamide $(4,-)$. CV conditions: $0.2 \mathrm{M}$ [TBA] $\mathrm{PF}_{6}$ solution of hfip, glassy carbon working electrode, and Pt counter electrode 


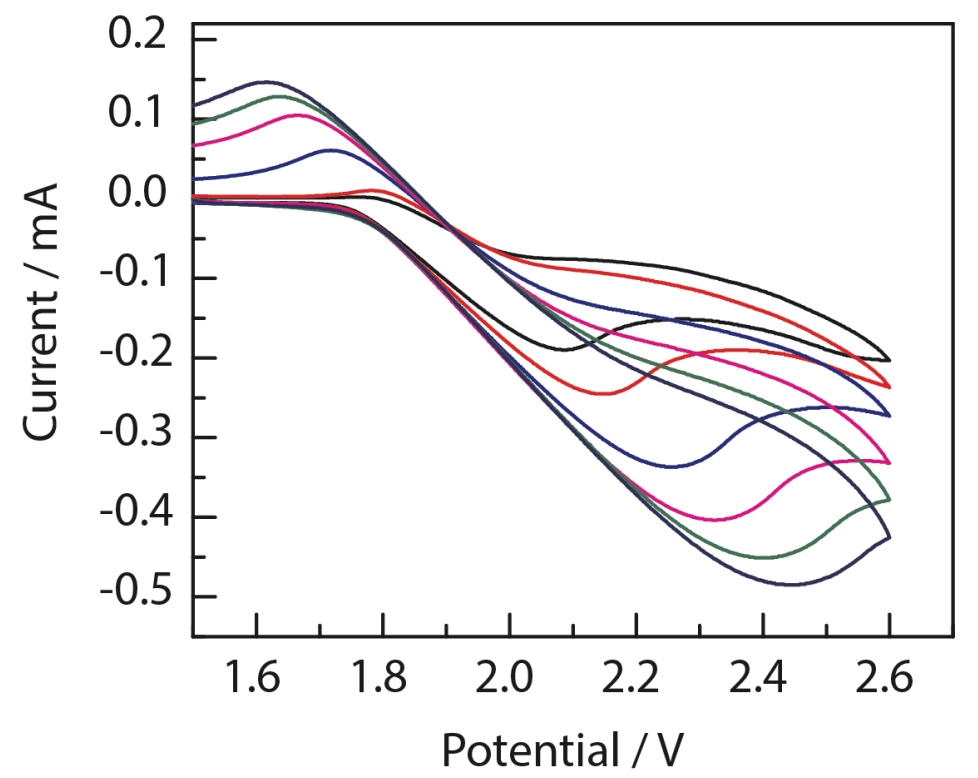

Figure S9. Increasing reversibility of 4-iodotoluene oxidation in hfip is observed in cyclic voltammograms collected with increasing scan rate: $0.05 \mathrm{~V} / \mathrm{s}(-), 0.1 \mathrm{~V} / \mathrm{s}(-), 0.25 \mathrm{~V} / \mathrm{s}(-$ ), $0.50 \mathrm{~V} / \mathrm{s} \mathrm{(-),} 0.75 \mathrm{~V} / \mathrm{s} \mathrm{(-),} \mathrm{and} 1.0 \mathrm{~V} / \mathrm{s} \mathrm{(-).} \mathrm{CV} \mathrm{conditions:} 10 \mathrm{mM}$ 4-iodotoluene, $0.2 \mathrm{M}$ $[\mathrm{TBA}] \mathrm{PF}_{6}$ solution of hfip, glassy carbon working electrode, and Pt counter electrode. 

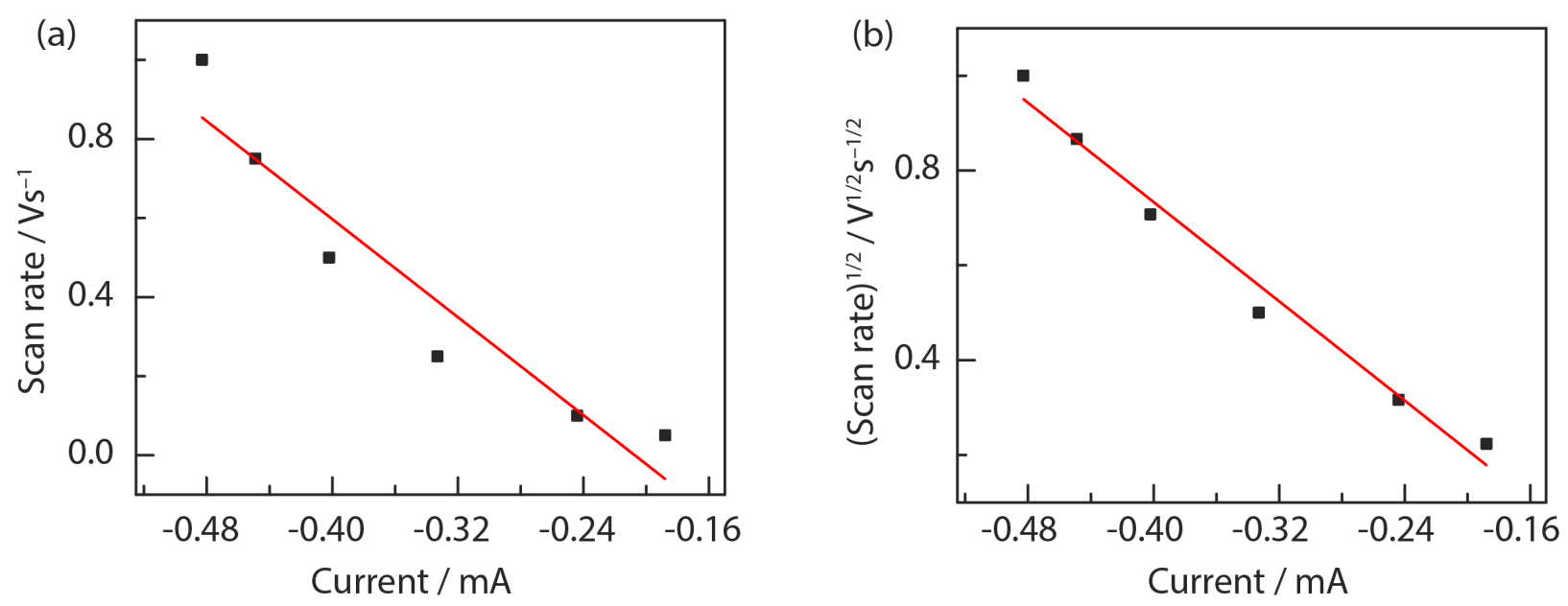

Figure S10. Plot of (a) scan rate vs peak anodic current ( $\mathrm{I}_{\mathrm{pa}}$ ) of 4 -iodotoluene $\left[\mathrm{R}^{2}=0.89\right]$ and (b) square root of scan rate vs. peak anodic current $\left(\mathrm{I}_{\mathrm{pa}}\right)\left[\mathrm{R}^{2}=0.98\right]$. The better fit for (b) suggests that no adsorption of aryl iodide was observed on the surface of the electrode. Data collected from Figure S9. 


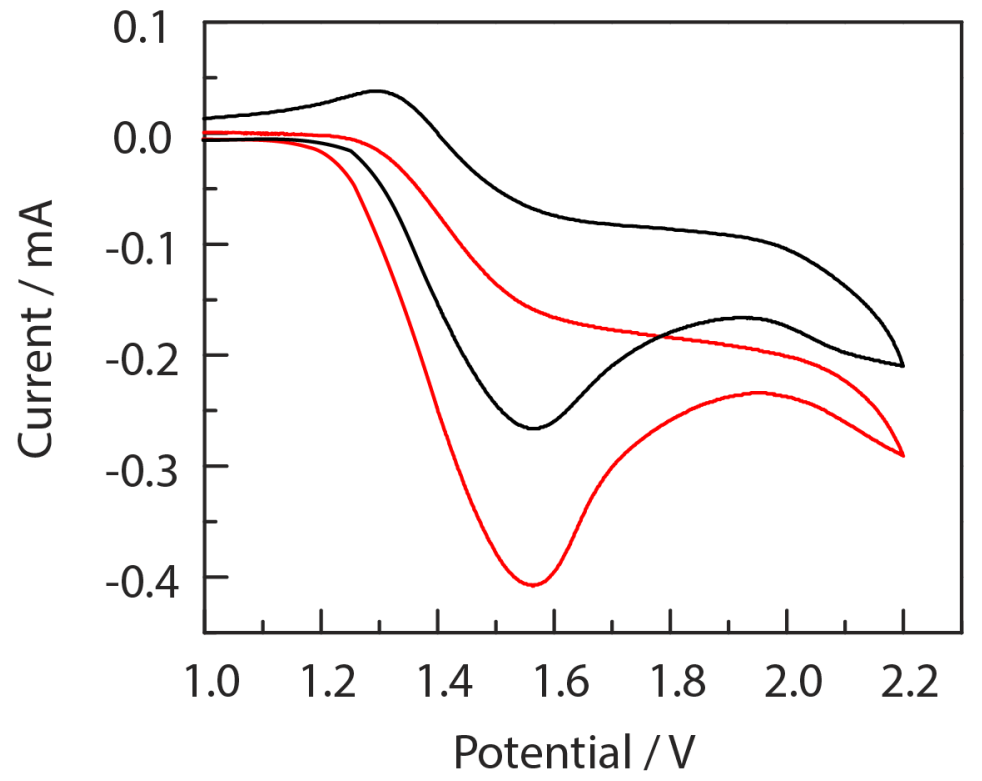

Figure S11. Cyclic voltammograms of 4-iodotoluene in a $0.2 \mathrm{M}$ [TBA] $\mathrm{PF}_{6}$ solution of hfip () and in presence of 2.0 equivalents of [TBA]OAc (-). CV conditions: $10 \mathrm{mM}$ 4-iodotoluene, $0.2 \mathrm{M}$ [TBA] $\mathrm{PF}_{6}$ glassy carbon working electrode, $\mathrm{Pt}$ counter electrode, and scan rate $=0.25$ $\mathrm{V} / \mathrm{s}$. 


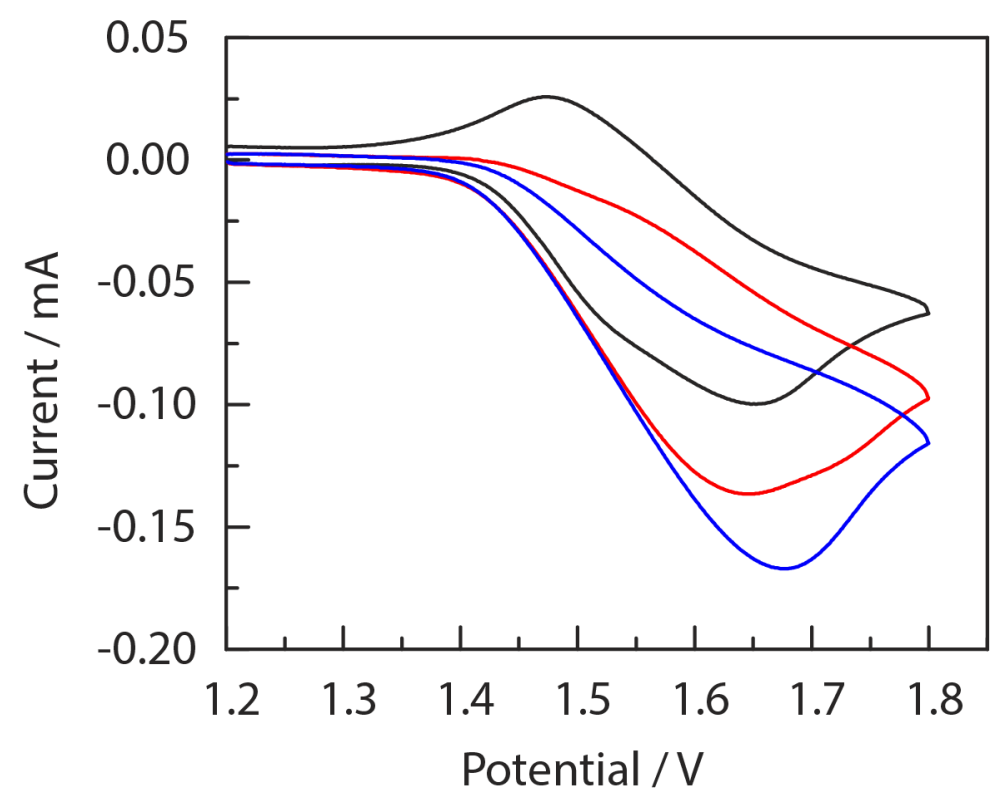

Figure S12. Cyclic voltammograms of 4-iodoanisole $(5 \mathrm{mM})$ in a $0.2 \mathrm{M}[\mathrm{TBA}] \mathrm{PF}_{6}$ solution of hfip (-), in presence of 1.0 equivalent of pyridine (-), and in the presence of 2.0 equivalent of pyridine $(-)$. CV conditions: glassy carbon working electrode, Pt counter electrode, and scan rate $=0.25 \mathrm{~V} / \mathrm{s}$.

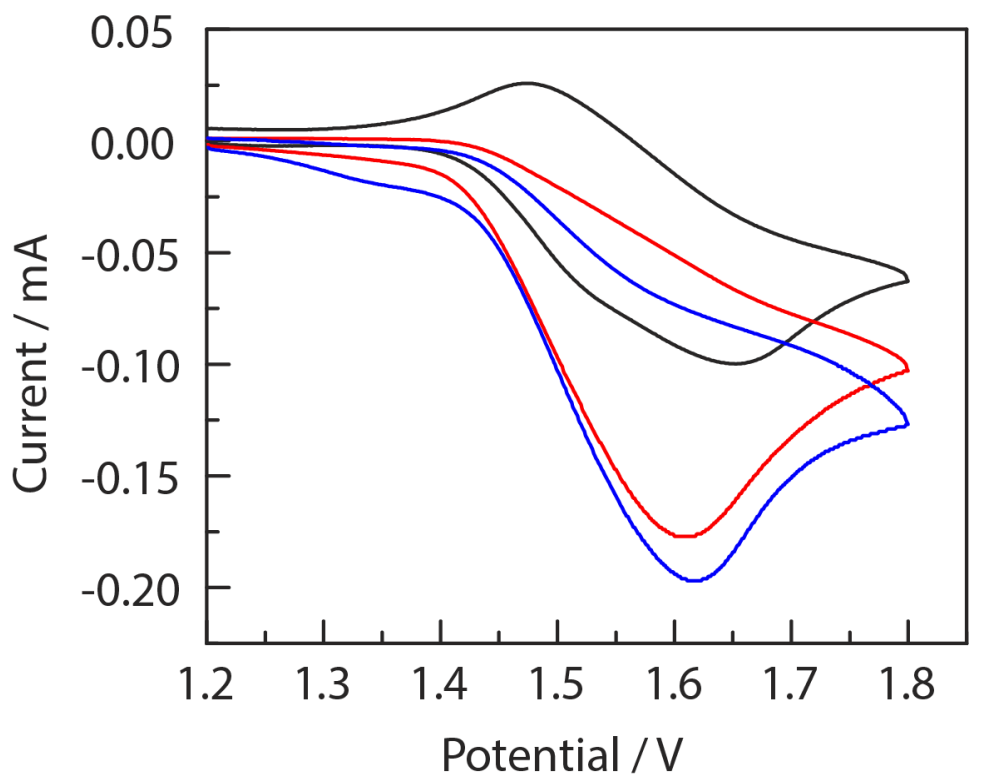

Figure S13. Cyclic voltammograms of 4-iodoanisole $(5 \mathrm{mM})$ in a $0.2 \mathrm{M}[\mathrm{TBA}] \mathrm{PF}_{6}$ solution of hfip (-), in presence of 1.0 equivalent of [TBA]CN $(-)$, and in the presence of 2.0 equivalent of [TBA]CN (-). CV conditions: glassy carbon working electrode, Pt counter electrode, and scan rate $=0.25 \mathrm{~V} / \mathrm{s}$. 


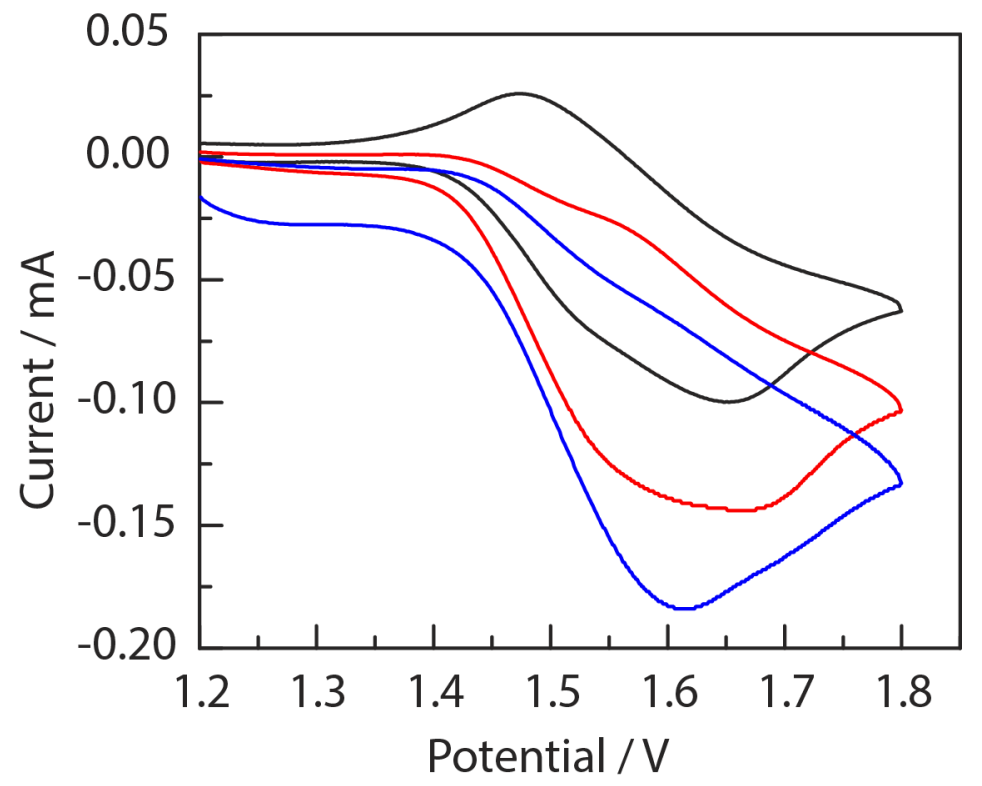

Figure S14. Cyclic voltammograms of 4-iodoanisole $(5 \mathrm{mM})$ in a $0.2 \mathrm{M}$ [TBA] $\mathrm{PF}_{6}$ solution of hfip (-), in presence of 1.0 equivalent of [TBA]TFA (-), and in the presence of 2.0 equivalent of [TBA]TFA $(-)$. CV conditions: glassy carbon working electrode, Pt counter electrode, and scan rate $=0.25 \mathrm{~V} / \mathrm{s}$.

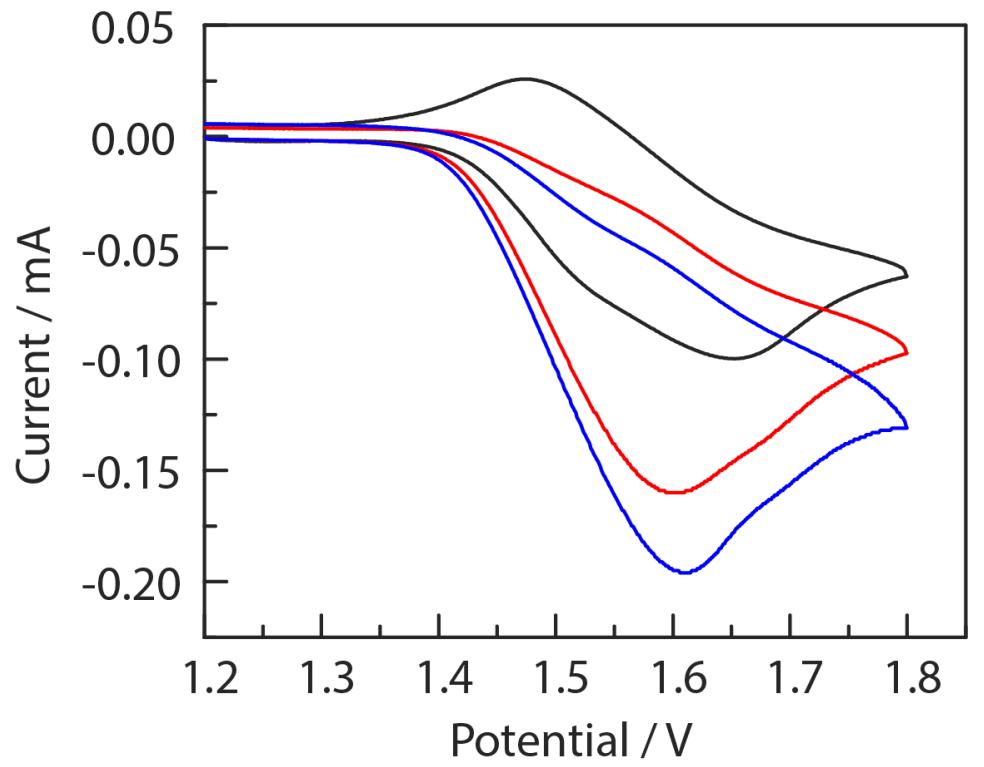

Figure S15. Cyclic voltammograms of 4-iodoanisole $(5 \mathrm{mM})$ in a $0.2 \mathrm{M}$ [TBA] $\mathrm{PF}_{6}$ solution of hfip (-), in presence of 1.0 equivalent of [TBA] $\cdot 3 \mathrm{H}_{2} \mathrm{O}(-)$, and in the presence of 2.0 equivalent of $[\mathrm{TBA}] \mathrm{F} \cdot 3 \mathrm{H}_{2} \mathrm{O}(-)$. $\mathrm{CV}$ conditions: glassy carbon working electrode, Pt counter electrode, and scan rate $=0.25 \mathrm{~V} / \mathrm{s}$. 


\section{Electrolysis and headspace analysis of [TBA]0Ac in acetonitrile}

$$
\begin{gathered}
\text { [TBA]OAc } \frac{\left[\mathrm{TBA}_{\mathrm{PFF}}\right.}{\mathrm{MeCN}, 23^{\circ} \mathrm{C}} \mathrm{CO}_{2}+\mathrm{CH}_{3}-\mathrm{CH}_{3}+\mathrm{CH}_{4} \\
\mathrm{CPE} 1.8 \mathrm{~V} \mathrm{v} \mathrm{Ag} / \mathrm{Ag}
\end{gathered}
$$

A $10-\mathrm{mL}$ glass vial was charged with tetrabutylammonium acetate $(120 \mathrm{mg}, 0.401 \mathrm{mmol}$, 1.00 equiv.), tetrabutylammonium hexafluorophosphate (390 mg, $1.01 \mathrm{mmol}, 2.52$ equiv.), and acetonitrile $(5.0 \mathrm{~mL})$ and was fitted with glassy carbon anode, platinum cathode, and $\mathrm{Ag}^{+} / \mathrm{Ag}$ reference electrode. A constant potential of $1.8 \mathrm{~V}$ vs. $\mathrm{Ag}^{+} / \mathrm{Ag}$ was applied to the reaction mixture and stirred at $23^{\circ} \mathrm{C}$ until $\sim 80 \mathrm{C}$ charge $(\sim 2.07 \mathrm{~F} / \mathrm{mol})$ was passed. The headspace of the reaction mixture was then analyzed by gas chromatography. Data are collected in Figure S16.

(a)

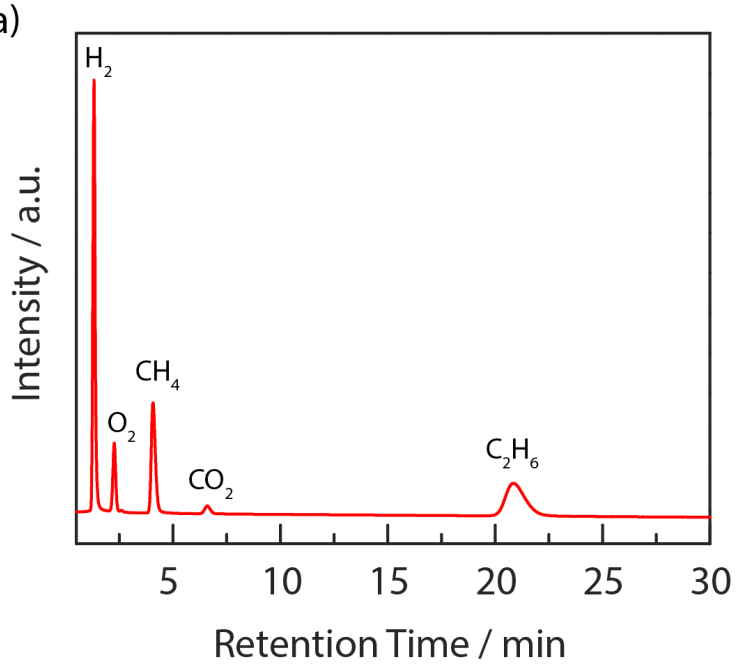

(b)

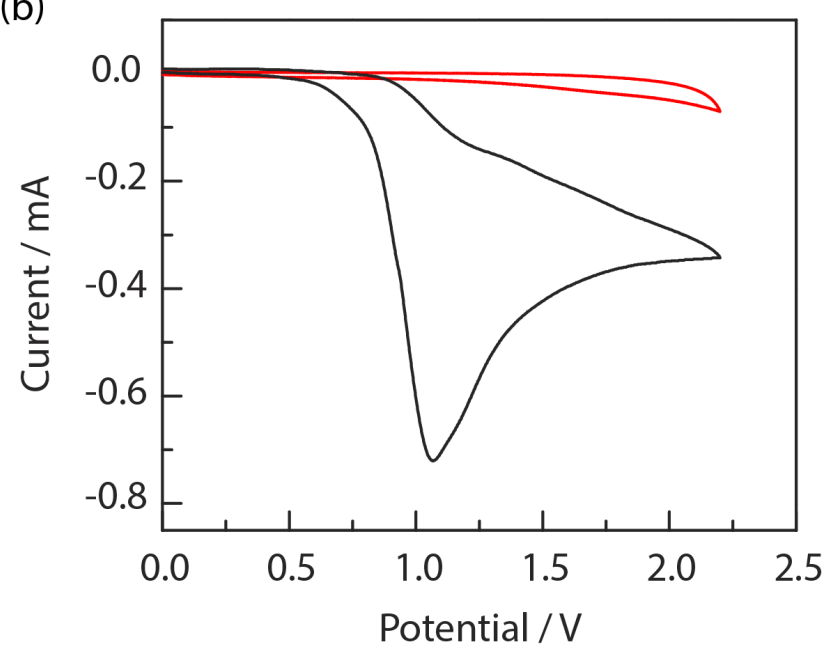

Figure S16. (a) Gas chromatogram of headspace of [TBA]OAc electrolysis in acetonitrile. Anodic oxidation of acetate leads to decarboxylation of initially formed acetoxy radical and produces methane $\left(\mathrm{CH}_{4}\right)$, carbon dioxide $\left(\mathrm{CO}_{2}\right)$ and ethane $\left(\mathrm{C}_{2} \mathrm{H}_{6}\right)$. Hydrogen gas is observed as the product of cathodic half-cell reaction. The oxygen $\left(\mathrm{O}_{2}\right)$ peak is due to the ambient conditions used for reaction set-up. (b) Comparison of cyclic voltammograms of $0.2 \mathrm{M}$ [TBA] $\mathrm{PF}_{6}$ solution in acetonitrile: before $(-)$ and after addition of [TBA]OAc $(-)$. CV conditions: $10 \mathrm{mM}[\mathrm{TBA}] \mathrm{OAc}$, glassy carbon working electrode, Pt counter electrode, scan rate $0.1 \mathrm{~V} / \mathrm{s}$. 


\section{Electrolysis and headspace analysis of [TBA]OAc in hfip}

$$
\begin{gathered}
\text { [TBA]OAc } \frac{\left[\mathrm{TBA}_{\mathrm{P}} \mathrm{PF}_{6}\right.}{\mathrm{hfip,} 23^{\circ} \mathrm{C}} \mathrm{H}_{2} \text { trace } \\
\mathrm{CPE} 1.8 \mathrm{~V} \mathrm{vs} \mathrm{Ag} / \mathrm{Ag}
\end{gathered}
$$

A $10-\mathrm{mL}$ glass vial was charged with tetrabutylammonium acetate $(120 \mathrm{mg}, 0.401 \mathrm{mmol}$, 1.00 equiv.), tetrabutylammonium hexafluorophosphate (390 mg, $1.01 \mathrm{mmol}, 2.52$ equiv.), and hfip $\left(5.0 \mathrm{~mL}\right.$ ) and was fitted with glassy carbon anode, platinum cathode, and $\mathrm{a} \mathrm{Ag}^{+} / \mathrm{Ag}$ reference electrode. A constant potential of $1.8 \mathrm{~V}$ vs. $\mathrm{Ag}^{+} / \mathrm{Ag}$ was applied to the reaction mixture and the reaction was stirred at $23{ }^{\circ} \mathrm{C}$ for $12 \mathrm{~h}$. No significant current was observed during this period and only $\sim 2 \mathrm{C}$ of charge was passed. The headspace of the reaction mixture was analyzed by gas chromatography. Data are collected in Figure S17.
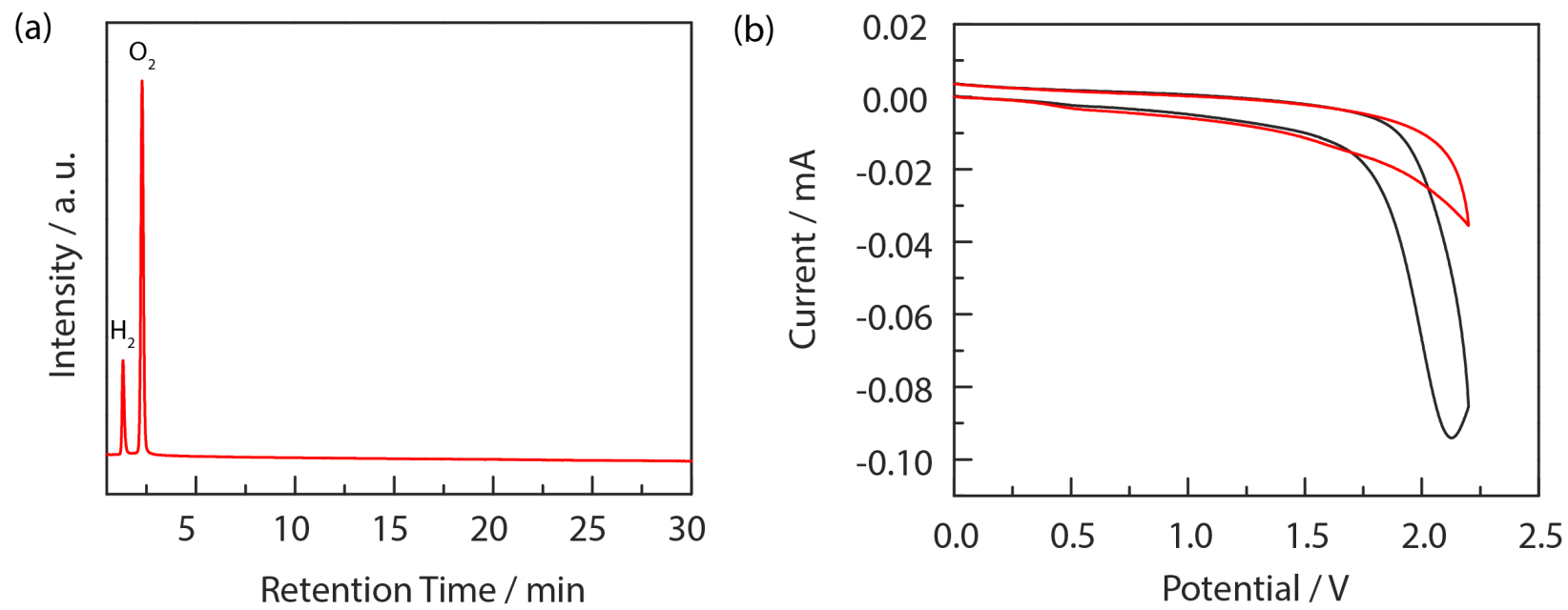

Figure S17. (a) Gas chromatogram of headspace of [TBA]OAc electrolysis in hfip. In hfip, anodic oxidation of acetate is suppressed and no products derived from decarboxylation are observed. The oxygen $\left(\mathrm{O}_{2}\right)$ peak is due to the ambient conditions used for reaction set-up. (b) Comparison of cyclic voltammograms of $0.2 \mathrm{M}$ [TBA] $\mathrm{PF}_{6}$ solution in acetonitrile: before $(-)$ and after addition of [TBA]OAc (-). CV conditions: $10 \mathrm{mM}$ [TBA]OAc, glassy carbon working electrode, $\mathrm{Pt}$ counter electrode, scan rate $0.1 \mathrm{~V} / \mathrm{s}$. 


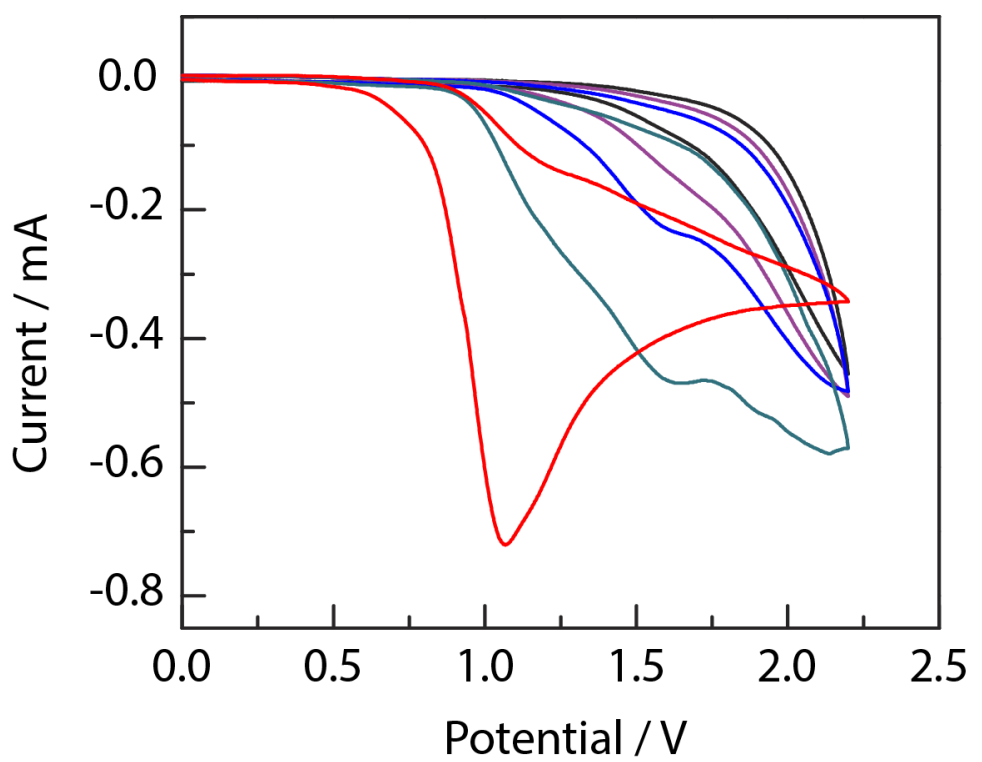

Figure S18. Effect of addition of hfip to the cyclic voltammogram of [TBA]OAc in $0.2 \mathrm{M}$ [TBA] $\mathrm{PF}_{6}$ solution of acetonitrile. $(-) 0$ equiv., $(-) 1$ equiv., $(-) 2$ equiv., $(-) 3$ equiv. and (-) 5 equiv. of hfip. CV conditions: glassy carbon working electrode, Pt counter electrode, scan rate $0.1 \mathrm{~V} / \mathrm{s}$. 


\section{Evaluation of H-bonding between hfip and [TBA]OAc}

Stock solutions ( $10 \mathrm{mM}$ ) of hfip and [TBA]OAc were prepared separately in $\mathrm{CD}_{3} \mathrm{CN}$. Ten NMR samples were prepared with different proportions of the hfip and [TBA]OAc solution so that the total concentration of ([hfip] + [[TBA]OAc]) for each sample was $10 \mathrm{mM}$ (Table S11). Each NMR sample was run at $23^{\circ} \mathrm{C}$ and the sample was allowed to equilibrate in the magnet for 5 min before the ${ }^{1} \mathrm{H}$ NMR spectrum was recorded. As shown in Figure S19, the resonance of the $3^{\circ}$ proton (adjacent to $\mathrm{OH}$ group) was shifted upfield as the mole fraction of hfip was increased. The Job plot, shown in Figure S20, was obtained plotting $\chi_{\text {hfip }} \times \Delta \delta_{\text {ppm }}$ with $\chi_{\text {hfip, }}$ indicating a 1:1 complex stoichiometry.

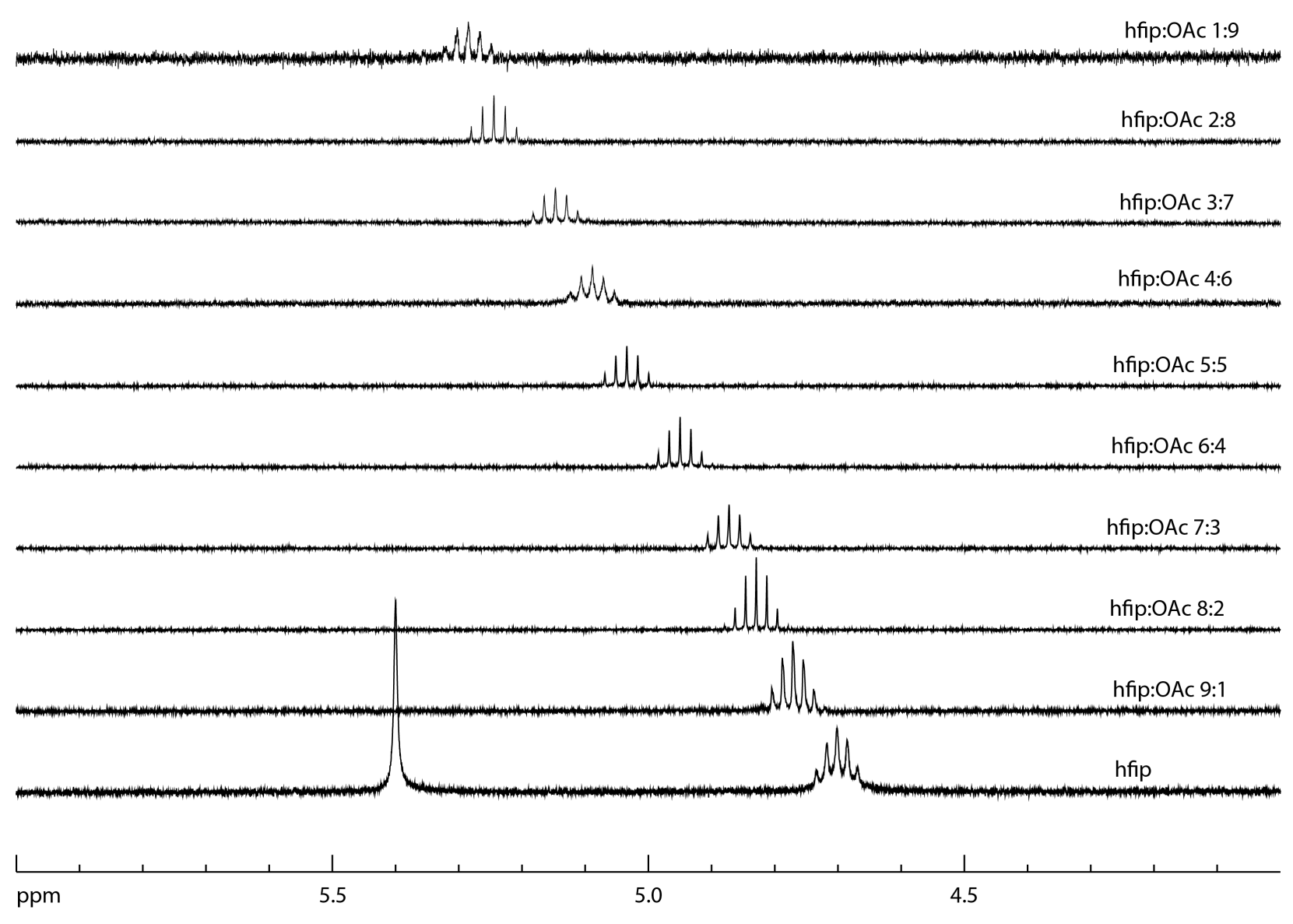

Figure S19. ${ }^{1} \mathrm{H}$ NMR spectra of hfip titration with $[\mathrm{TBA}] \mathrm{OAc}$ in $\mathrm{CD}_{3} \mathrm{CN}$. In this plot, change of chemical shift of $3^{\circ}$ proton adjacent to $\mathrm{OH}$ group is measured as a function of [TBA]OAc concentration. 
Table S11. Data for the Job plot performed by ${ }^{1} \mathrm{H}$ NMR titration in $\mathrm{CD}_{3} \mathrm{CN}$.

\begin{tabular}{ccccccccc} 
Entry & $\begin{array}{c}\text { hfip } \\
(\mu \mathrm{L})\end{array}$ & $\begin{array}{c}{[\mathrm{TBA}] \mathrm{OAc}} \\
(\mu \mathrm{L})\end{array}$ & $\begin{array}{c}\text { hfip } \\
(\mathrm{mM})\end{array}$ & $\begin{array}{c}{[\mathrm{TBA}] \mathrm{OAc}} \\
(\mathrm{mM})\end{array}$ & $\chi_{\text {hfip }}$ & $\begin{array}{c}\delta \\
(\mathrm{ppm})\end{array}$ & $\begin{array}{c}\Delta \delta \\
(\mathrm{ppm})\end{array}$ & $\chi_{\text {hfip }} \times \Delta \delta_{\mathrm{ppm}}$ \\
\hline 1 & 1000 & 0 & 10 & 0 & 1 & 4.70 & 0 & - \\
2 & 900 & 100 & 9 & 1 & 0.9 & 4.77 & 0.07 & 0.063 \\
3 & 800 & 200 & 8 & 2 & 0.8 & 4.83 & 0.13 & 0.102 \\
4 & 700 & 300 & 7 & 3 & 0.7 & 4.87 & 0.17 & 0.119 \\
5 & 600 & 400 & 6 & 4 & 0.6 & 4.95 & 0.25 & 0.150 \\
6 & 500 & 500 & 5 & 5 & 0.5 & 5.03 & 0.33 & 0.165 \\
7 & 400 & 600 & 4 & 6 & 0.4 & 5.09 & 0.39 & 0.156 \\
8 & 300 & 700 & 3 & 7 & 0.3 & 5.15 & 0.45 & 0.135 \\
9 & 200 & 800 & 2 & 8 & 0.2 & 5.24 & 0.54 & 0.108 \\
10 & 100 & 900 & 1 & 9 & 0.1 & 5.29 & 0.58 & 0.058
\end{tabular}

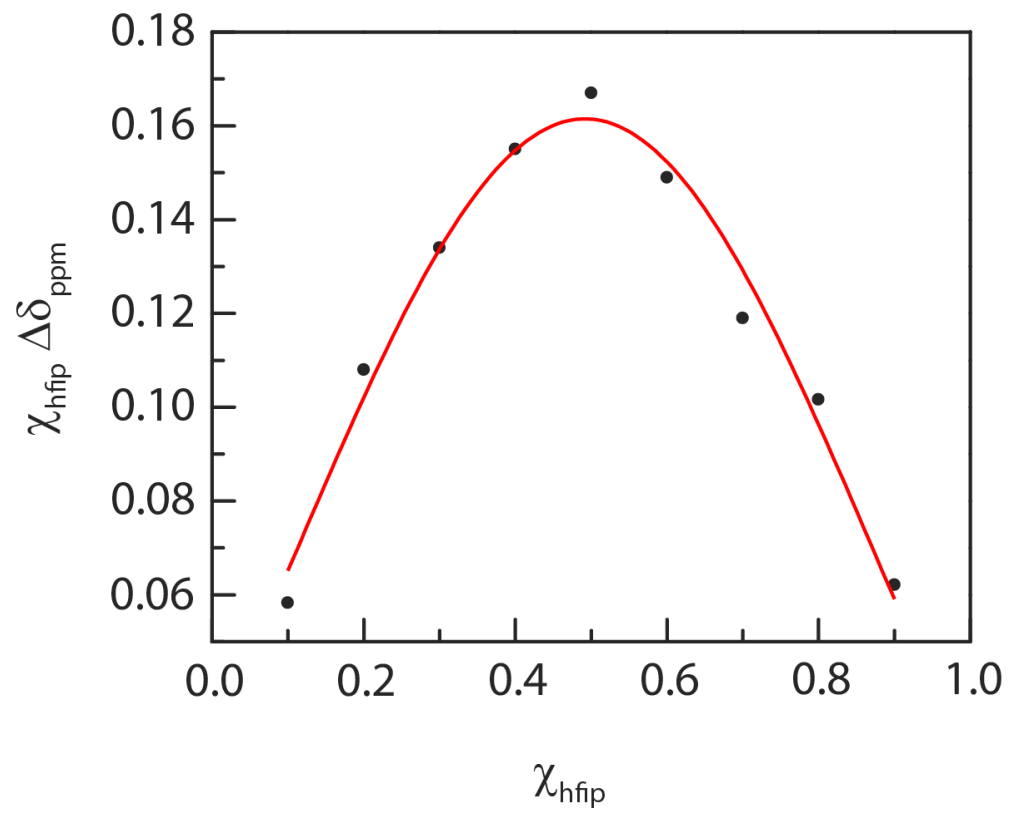

Figure S20. Job plot constructed from the ${ }^{1} \mathrm{H}$ NMR data shown in Figure S19. The maximum at $\sim 0.5$ indicates a 1:1 interaction between hfip and [TBA]OAc. 


\section{Determination of the $\mathrm{K}_{\mathrm{eq}}$ for hfip binding to [TBA] $\mathrm{AAc}$}

In order to determine the equilibrium constant $\left(\mathrm{K}_{\mathrm{eq}}\right)$ for acetate binding to hfip, an NMR tube was charged with mesitylene ( $3.2 \mathrm{mg}, 0.027 \mathrm{mmol}$ ), hfip (5.0 $\mu \mathrm{L}, 0.048 \mathrm{mmol})$, and $\mathrm{CD}_{3} \mathrm{CN}$ $(0.50 \mathrm{~mL})$. The sample was allowed to equilibrate in the magnet for $5 \mathrm{~min}$ before the ${ }^{1} \mathrm{H}$ NMR spectrum was recorded, and the spectrum was remeasured after $30 \mathrm{~min}$ to ensure that equilibrium had been reached. In a 20-mL scintillation vial a stock solution of [TBA] OAc (80 $\mathrm{mg}, 0.27 \mathrm{mmol})$ in $\mathrm{CD}_{3} \mathrm{CN}(0.50 \mathrm{~mL})$ was prepared. To the solution in NMR tube, $20 \mu \mathrm{L}$ of [TBA]OAc stock solution was added and the corresponding ${ }^{1} \mathrm{H}$ NMR spectrum was recorded. Each NMR sample was run at $23^{\circ} \mathrm{C}$ and the amount of hfip and [TBA]OAc in a given sample was determined using mesitylene as the internal standard. As shown in Figure S21, the resonance of the $3^{\circ}$ proton (adjacent to $\mathrm{OH}$ group) was shifted downfield as the mole fraction of acetate anion was increased. The Job plot, shown in Figure S22, was obtained plotting $\chi_{\text {hfip }}$ $\times \Delta \delta_{\text {ppm }}$ with $\chi_{\text {hfip }}$, indicating a 1:1 complex stoichiometry.

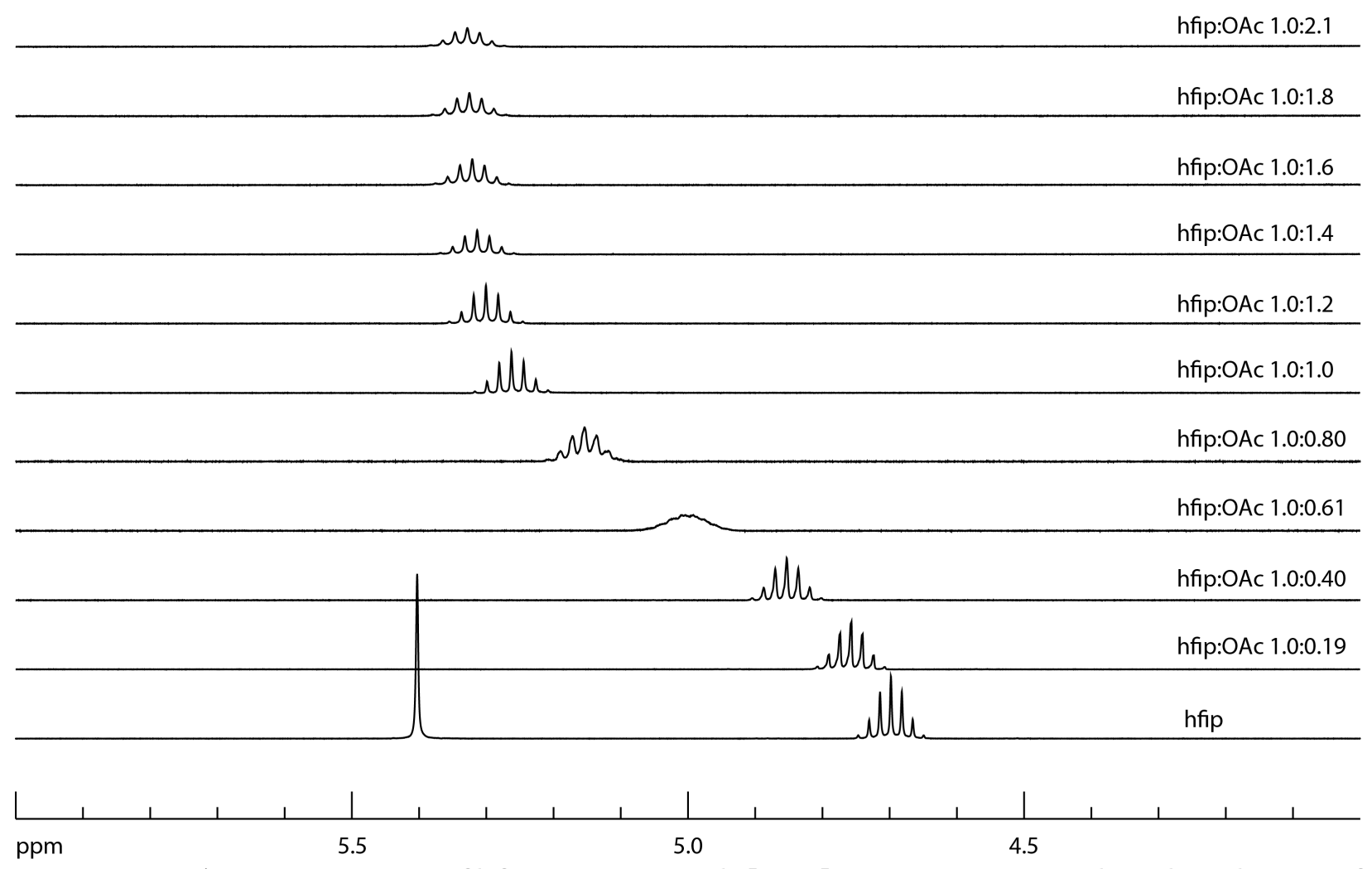

Figure S21. ${ }^{1} \mathrm{H}$ NMR spectra of hfip titration with [TBA] $0 A c$ in $\mathrm{CD}_{3} \mathrm{CN}$. In this plot, change of chemical shift of $3^{\circ}$ proton adjacent to $\mathrm{OH}$ group is measured as a function of [TBA]OAc concentration. 
Table S12. Data for the Job plot performed by ${ }^{1} \mathrm{H}$ NMR titration in $\mathrm{CD}_{3} \mathrm{CN}$.

\begin{tabular}{ccccccc} 
Entry & hfip (mM) & {$[\mathrm{TBA}] \mathrm{MAc}(\mathrm{mM})$} & $\chi_{\text {hfip }}$ & $\delta(\mathrm{ppm})$ & $\Delta \delta(\mathrm{ppm})$ & \\
\hline 1 & 0.0489 & - & 1.00 & 4.70 & 0 & - \\
2 & 0.0489 & 0.00932 & 0.840 & 4.78 & 0.08 & 0.0672 \\
3 & 0.0489 & 0.0198 & 0.712 & 4.85 & 0.15 & 0.107 \\
4 & 0.0489 & 0.0298 & 0.622 & 5.01 & 0.31 & 0.193 \\
5 & 00.0489 & 0.0394 & 0.554 & 5.15 & 0.45 & 0.249 \\
6 & 0.0489 & 0.0515 & 0.488 & 5.26 & 0.56 & 0.273 \\
7 & 0.0489 & 0.0602 & 0.449 & 5.3 & 0.6 & 0.269 \\
8 & 0.0489 & 0.0702 & 0.411 & 5.31 & 0.61 & 0.251 \\
9 & 0.0489 & 0.0808 & 0.377 & 5.32 & 0.62 & 0.234 \\
10 & 0.0489 & 0.0923 & 0.3467 & 5.33 & 0.63 & 0.218 \\
11 & 0.0489 & 0.101 & 0.326 & 5.33 & 0.63 & 0.205
\end{tabular}

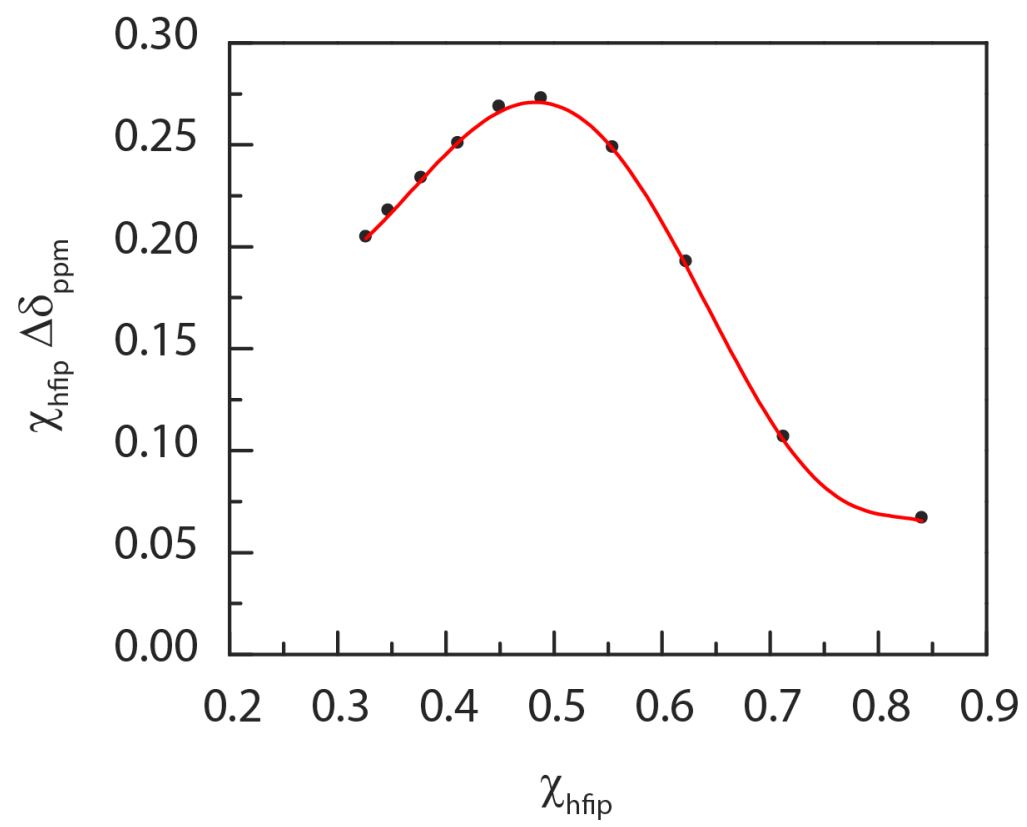

Figure S22. Job plot constructed from the ${ }^{1} \mathrm{H}$ NMR data shown in Figure S21. The maximum at $\sim 0.5$ indicates a 1:1 interaction between hfip and [TBA]OAc. 
The equilibrium constant for the formation of an adduct between hfip and [TBA]OAc was calculated from Eq. $\mathrm{S} 1^{39}$

$$
K_{e q}=\frac{\Delta \delta_{o b s}}{\left(\Delta \delta_{C A}-\Delta \delta_{o b s}\right)\left([H F I P]-\frac{[x x] \Delta \delta_{o b s}}{\Delta \delta_{C A}}\right)}
$$

where

$\Delta \delta_{o b s}=$ difference between the chemical shifts of neat hfip and that of in presence of $[\mathrm{TBA}] \mathrm{OAc}$

$\Delta \delta_{C A}=$ change in chemical shift between the completely complexed and uncomplexed molecule

For a range of possible $\Delta \delta_{C A}$ values, $\mathrm{K}_{\mathrm{eq}}$ was determined following the equation $\mathrm{S} 1$ for entries 8-11 in Table S11 and the trial $K_{\text {eq }}$ vs. $\Delta \delta_{C A}$ was plotted for each concentration shown in Figure S22.

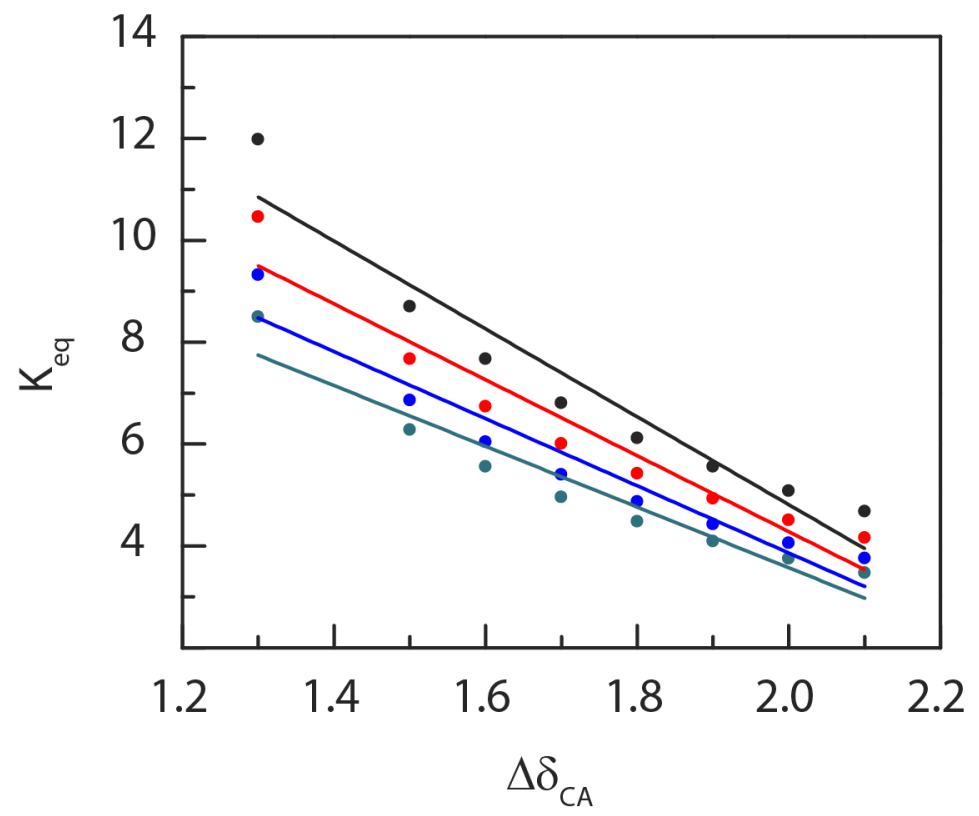

Figure S23. Linear fitting of $\mathrm{K}_{\mathrm{eq}}$ vs. $\Delta \delta_{C A}$ for solutions $8(-), 9(-), 10(-)$, and $11(-)$.

The equations for the best-fitted linear curve from the Figure S23 were listed below:

$y=22.06-8.62 x$ for solution $8(-) ; \mathrm{R}^{2}=0.92$

$y=19.19-7.46 x$ for solution $9(-) ; \mathrm{R}^{2}=0.91$

Eq. (S2)

$y=17.04-6.59 x$ for solution $10(-) ; \mathrm{R}^{2}=0.92$

Eq. (S3)

$y=15.49-5.96 x$ for solution $11(-) ; \mathrm{R}^{2}=0.93$

Eq. (S4)

Eq. (S5)

Since the $\mathrm{K}_{\mathrm{eq}}$ at a given temperature is constant for all the solutions, the value of $\Delta \delta_{C A}$ and $\mathrm{K}_{\mathrm{eq}}$ was determined solving Eq. S2-S4. The value of $\Delta \delta_{C A}$ was found to be $2.47 \mathrm{ppm}$. and the $\mathrm{K}_{\text {eq }}$ for hfip hydrogen-bonding with [TBA]OAc was determined to be $0.767 \pm 0.002$ at $23^{\circ} \mathrm{C}$. 


\section{Additional Data}

Table S13. Optimization of the electrochemical oxidation of 4-iodotoluene. Reaction conditions: $0.20 \mathrm{mmol}$ of 4-iodotoluene, $0.2 \mathrm{M}$ [TBA] $\mathrm{PF}_{6}, 5.0 \mathrm{~mL}$ of hfip, glassy carbon anode, and Pt cathode.

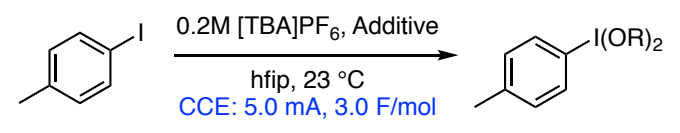

\begin{tabular}{ccccc}
\hline Entry & Anode & Cathode & Additive & Yield (\%) \\
\hline 1 & Glassy Carbon & $\mathrm{Pt}$ & [TBA]OAc (2 eq.) & 60 \\
2 & Glassy Carbon & $\mathrm{Pt}$ & AcOH (5 eq.) & 8 \\
3 & Glassy Carbon & $\mathrm{Pt}$ & - & 40 (hfip adduct) \\
4 & $\mathrm{Pt}$ & $\mathrm{Pt}$ & {$[\mathrm{TBA}] \mathrm{OAc}$ (2 eq.) } & 28 \\
5 & Graphite & $\mathrm{Pt}$ & {$[\mathrm{TBA}] \mathrm{OAc}$ (2 eq.) } & 32 \\
6 & BDC & $\mathrm{Pt}$ & {$[\mathrm{TBA}] \mathrm{OAc}$ (2 eq.) } & 41 \\
\hline
\end{tabular}


Table S14. Optimization of aryl iodide catalyst. Reaction conditions: $0.20 \mathrm{mmol}$ of substrate, $0.05 \mathrm{mmol}$ of catalyst, $0.40 \mathrm{mmol}$ [TBA]OAc, $0.2 \mathrm{M}$ [TBA] $\mathrm{PF}_{6}, 5.0 \mathrm{~mL}$ of hfip, glassy carbon anode, and Pt cathode.

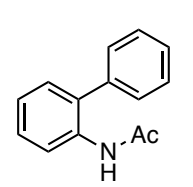

$1 \mathrm{a}$

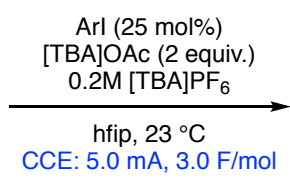

ArI

4-iodotoluene

4-iodoanisole iodobenzene 2,6-dimethoxyiodobenzene

2,4-dimethoxyiodobenzene

1,2-diiodobenzene

2 -iodobenzoic acid

2-(2-iodophenyl)propan-2-ol

9 1,1,1,3,3,3-hexafluoro-2-(2-iodophenyl)propan-2-ol
Yield (\%) 40 55

22

27

37

34

27

37

34 
Table S15. Optimization of catalyst loading and additives. Reaction conditions: $0.20 \mathrm{mmol}$ of substrate, $0.05 \mathrm{mmol}$ of catalyst, additive $0.20 \mathrm{mmol}, 0.40 \mathrm{mmol}$ [TBA]OAc, $0.2 \mathrm{M}$ [TBA] $\mathrm{PF}_{6}, 5.0 \mathrm{~mL}$ of hfip, glassy carbon anode, and Pt cathode.

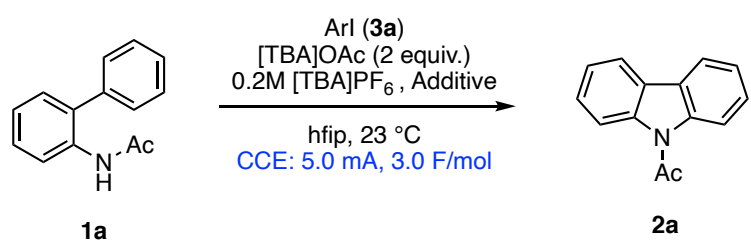

\begin{tabular}{cccc}
\hline Entry & ArI Loading & Additive & Yield(\%) \\
\hline 1 & 1 equiv. & - & 62 \\
2 & $25 \mathrm{~mol} \%$ & - & 57 \\
3 & $10 \mathrm{~mol} \%$ & - & 46 \\
4 & $5 \mathrm{~mol} \%$ & - & 35 \\
5 & $25 \mathrm{~mol} \%$ & $\mathrm{CH}_{2} \mathrm{Cl}_{2}$ & 28 \\
6 & $25 \mathrm{~mol} \%$ & $\mathrm{MeCN}^{2}$ & trace \\
7 & $25 \mathrm{~mol} \%$ & $\mathrm{Cs}_{2} \mathrm{O}_{3}$ & 56 \\
8 & $25 \mathrm{~mol} \%$ & $\mathrm{BF}_{3} \cdot \mathrm{OEt}_{2}$ & 46 \\
9 & $25 \mathrm{~mol} \%$ & $\mathrm{CF}_{3} \mathrm{COOH}$ & trace \\
\hline
\end{tabular}




\section{Control reaction for iodide mediated intramolecular $\mathrm{C}-\mathrm{N}$ coupling.}

To exclude the possibility of catalyst decomposition mediated reaction chemistry (via iodide oxidiation), background reaction with [TBA]I in place of 4-iodoanisole was attempted for the parent reaction (1a).
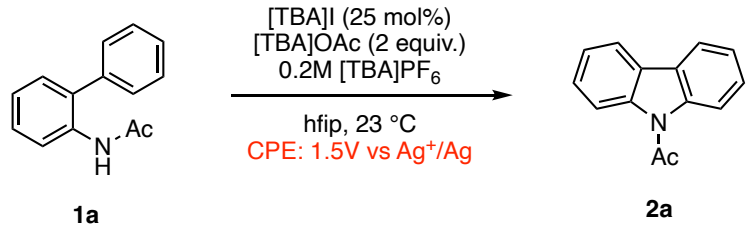

A 10 -mL glass vial was charged with $N$-([1,1'-biphenyl $]-2-y l)$ acetamide $(\mathbf{1 a}, 0.201 \mathrm{mmol}$, 1.00 equiv.), tetrabutylammonium iodide $(18.5 \mathrm{mg}, 0.05 \mathrm{mmol}, 25.0 \mathrm{~mol} \%)$, tetrabutylammonium acetate (121 $\mathrm{mg}, 0.401 \mathrm{mmol}, 1.99$ equiv.), tetrabutylammonium hexafluorophosphate $(390 \mathrm{mg}, 1.01 \mathrm{mmol}, 5.02$ equiv.), and hfip $(5.0 \mathrm{~mL})$. The reaction vessel was fitted with glassy carbon anode, platinum cathode, and $\mathrm{Ag}+\mathrm{Ag}$ reference electrode. A constant potential of $1.5 \mathrm{~V}$ vs $\mathrm{Ag}+/ \mathrm{Ag}$ was applied to the reaction mixture, which was stirred at $23{ }^{\circ} \mathrm{C}$. Due to low current densities the reaction was run until $45 \mathrm{C}$ charge $(\sim 2.33 \mathrm{~F} / \mathrm{mol})$ was passed, approximately $15 \mathrm{~h}$. Mesitylene was added directly to the crude reaction mixture as an internal standard and the yield was analyzed by ${ }^{1} \mathrm{H} \mathrm{NMR} \mathrm{in} \mathrm{CDCl}_{3}$. The yield was found to be 35\% with Faradaic efficiency of 30\%.

The yield of 35\% carbazole product (2a) was obtained, which is significantly lower than the $76 \%$ yield of optimized reaction condition. Though the Faradaic efficiency of $36 \%$ for [TBA]I is comparable with that of reaction with 4-iodoanisole (40\%), the current density for [TBA]I reaction was significantly lower than aryl iodide catalyzed reaction $45 \mathrm{C}$ of charge were passed for [TBA]I in $15 \mathrm{~h}$ whereas $80 \mathrm{C}$ of charge is passed with 4-iodoansole in 7-8h). In addition to this observation, high recovery of 4-iodoanisole $(\sim 95 \%)$ from parent reaction condition excludes the possibility of catalyst decomposition mediated chemistry. 


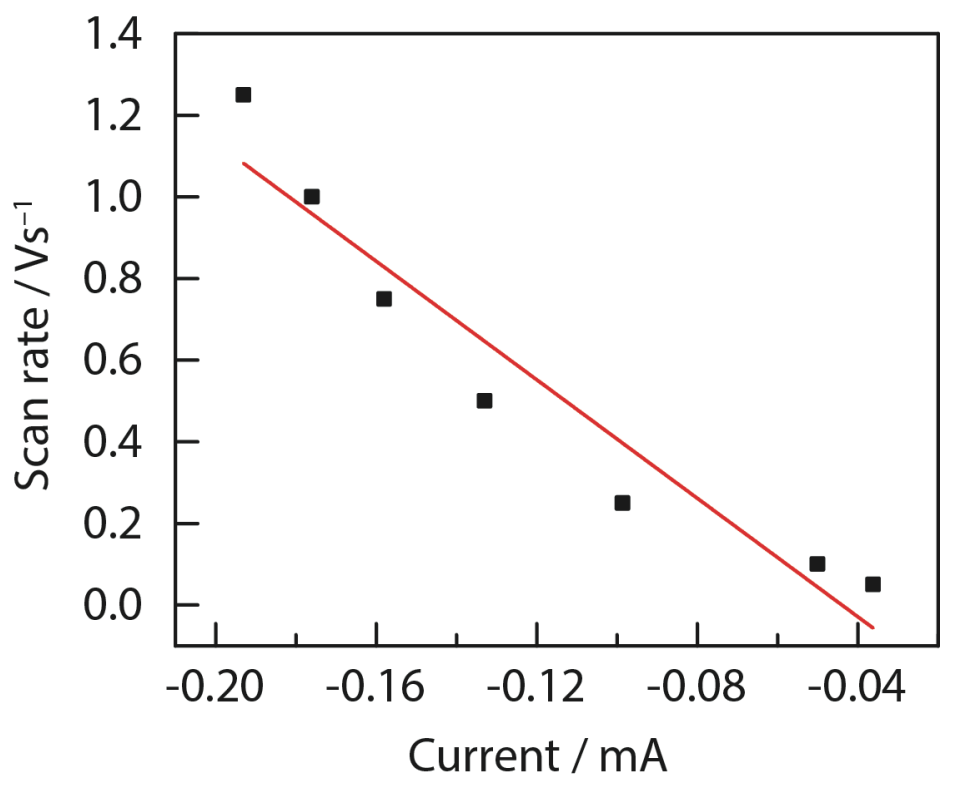

Figure S24. Plot of scan rate vs peak anodic current $\left(I_{p a}\right)$ of 4-iodoanisole $\left[R^{2}=0.91\right]$.The better fit for Figure 5a (inset) suggests that no adsorption of aryl iodide was observed on the surface of the electrode. Data collected from Figure $5 \mathrm{a}$. 

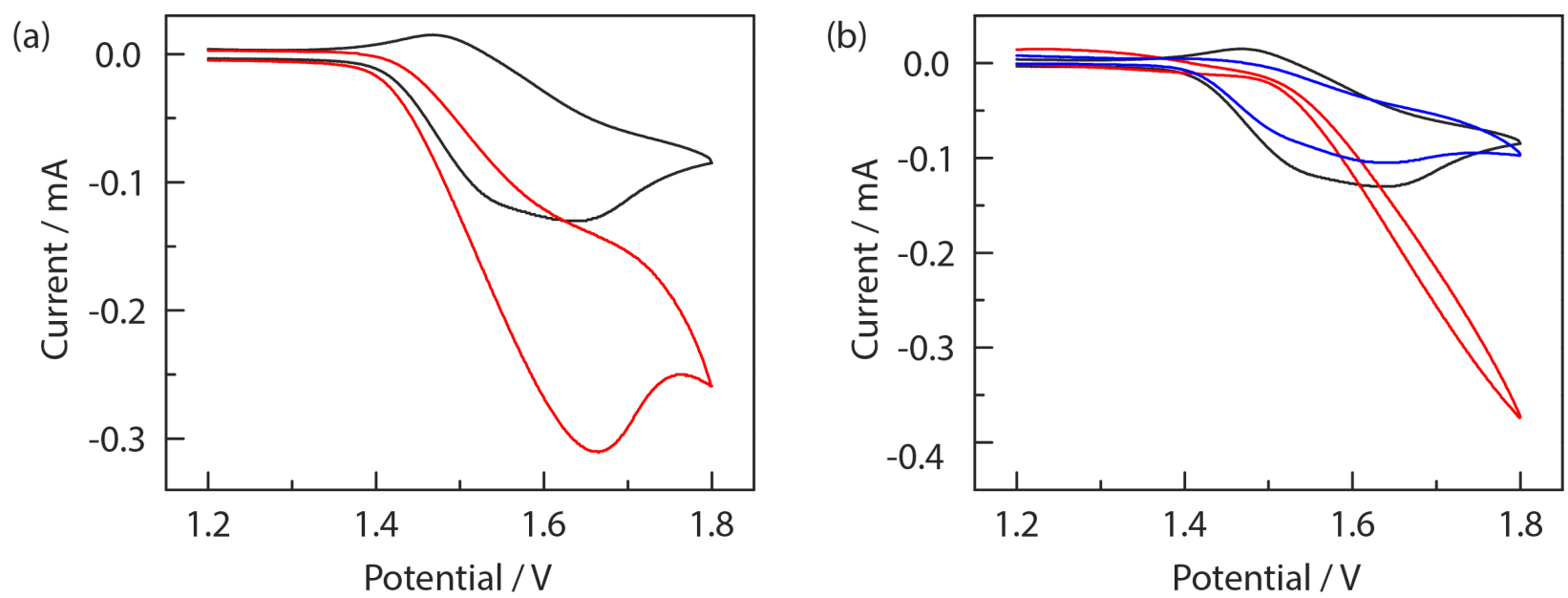

Figure S25. Comparison of current response with the addition of either (a) acetate, or (b) substrate (1a). (a) Cyclic voltammogram of 4-iodoanisole (3a) (-) and 3a with [TBA]OAc $(-)$. (b) Cyclic voltammogram of 4-iodoanisole (3a) (-), compound 1a $(-)$, and 3a with 1a (一). The increased current with addition of [TBA]OAc is consistent with [TBA]OAc trapping an electrochemically generated iodanyl radical. $\mathrm{CV}$ conditions: $0.2 \mathrm{M}$ [TBA] $\mathrm{PF}_{6}$ solution of hfip, glassy carbon working electrode, Pt counter electrode, and scan rate $=0.25 \mathrm{~V} / \mathrm{s}$. 


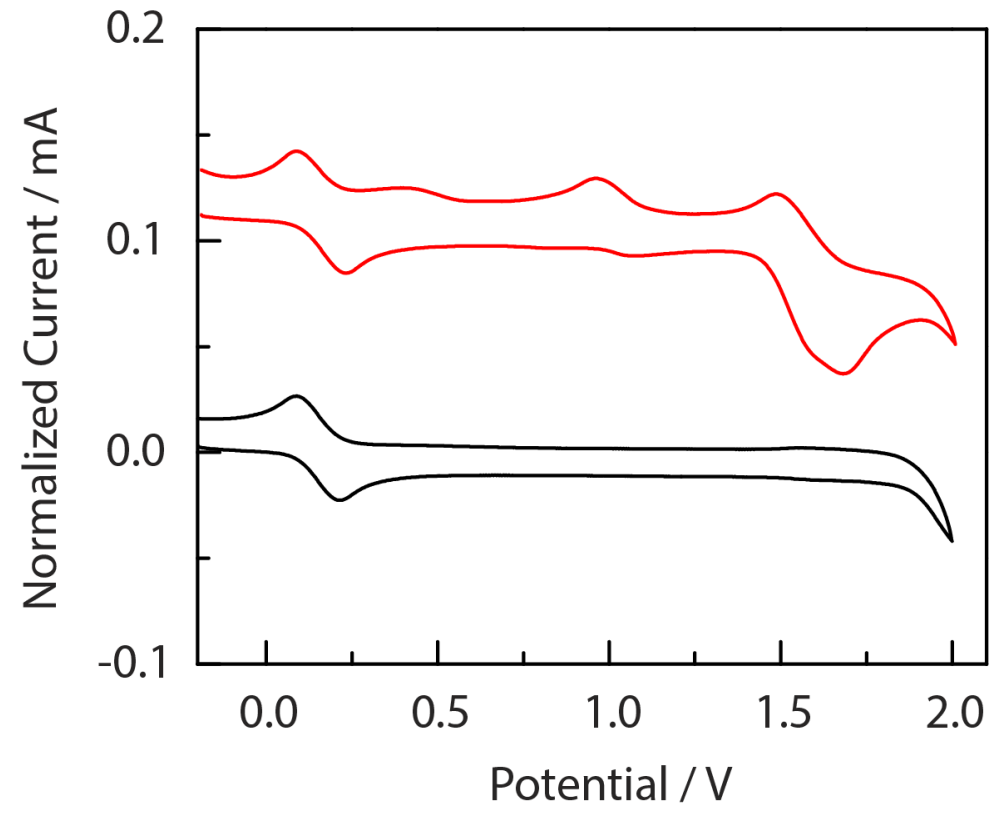

Figure S26. Comparison of peak separation between ferrocene and 4-iodoanisole in a $0.2 \mathrm{M}$ [TBA] $\mathrm{PF}_{6}$ solution of hfip. CV conditions: glassy carbon working electrode, Pt counter electrode, and scan rate $=0.25 \mathrm{~V} / \mathrm{s}$. 


\section{Calculation of Faradaic efficiency for various substrates}

The Faradaic efficiency of the described reactions are calculated using the following formula:

$$
\text { Faradaic effciency }=\frac{Q_{\text {theoretical }}}{Q_{\text {experimental }}} \times \frac{\text { reaction yield }}{100} \%
$$

Where,

$Q_{\text {theoretical }}=$ amount of charge required for the reaction for $100 \%$ efficiency $(2.0 \mathrm{~F} / \mathrm{mol})$

$Q_{\text {experimental }}=$ amount of charge passed through the reaction $(4.15 \mathrm{~F} / \mathrm{mol})$

reaction yield $=$ respective yield of product in consideration

Table S16. Comparison of Faradaic efficiency with selected publications in recent years on electrocatalytic substrate functionalization.

\begin{tabular}{lll}
\hline Entry & Reference & Faradaic Efficiency \\
\hline $\mathbf{1}$ & ACS Catal. 2019, 9, 746-754 & $27-90 \%$ \\
$\mathbf{2}$ & J. Am. Chem. Soc. 2018, $140,2438-2441$ & $<20 \%$ \\
$\mathbf{3}$ & J. Am. Chem. Soc. 2017, 139, 7448-7451 & $<15 \%$ \\
$\mathbf{4}$ & Nature 2019, 573, 398 & $<30 \%$ \\
$\mathbf{5}$ & J. Am. Chem. Soc. 2019, 141, 6392-6402 & $<30 \%$ \\
$\mathbf{6}$ & Angew. Chem. Int. Ed. 2017, 56, 13088-13093 & $48 \%$ \\
$\mathbf{7}$ & Angew. Chem., Int. Ed. 2010, 49, 129-133 & $<21 \%$ \\
$\mathbf{8}$ & J. Am. Chem. Soc. 2017, 139, 2956-2959 & $17-55 \%$ \\
$\mathbf{9}$ & J. Am. Chem. Soc. 2007, $129,6680-6681$ & $26-60 \%$ \\
$\mathbf{1 0}$ & J. Am. Chem. Soc. 2018, $140,11487-11494$ & $<15 \%$ \\
$\mathbf{1 1}$ & J. Am. Chem. Soc. 2015, 137, 9816-9819 & $35-51 \%$ \\
$\mathbf{1 2}$ & J. Am. Chem. Soc. 2007, $129,2246-2247$ & $42-60 \%$ \\
$\mathbf{1 3}$ & J. Am. Chem. Soc. 2009, $131,11310-11311$ & $<30 \%$ \\
$\mathbf{1 4}$ & J. Org. Chem. 1994, 59, 7190-7192 & $30-44 \%$ \\
$\mathbf{1 5}$ & This work & $21-40 \%$ \\
\hline
\end{tabular}




\section{E. NMR Spectra of New Compounds}
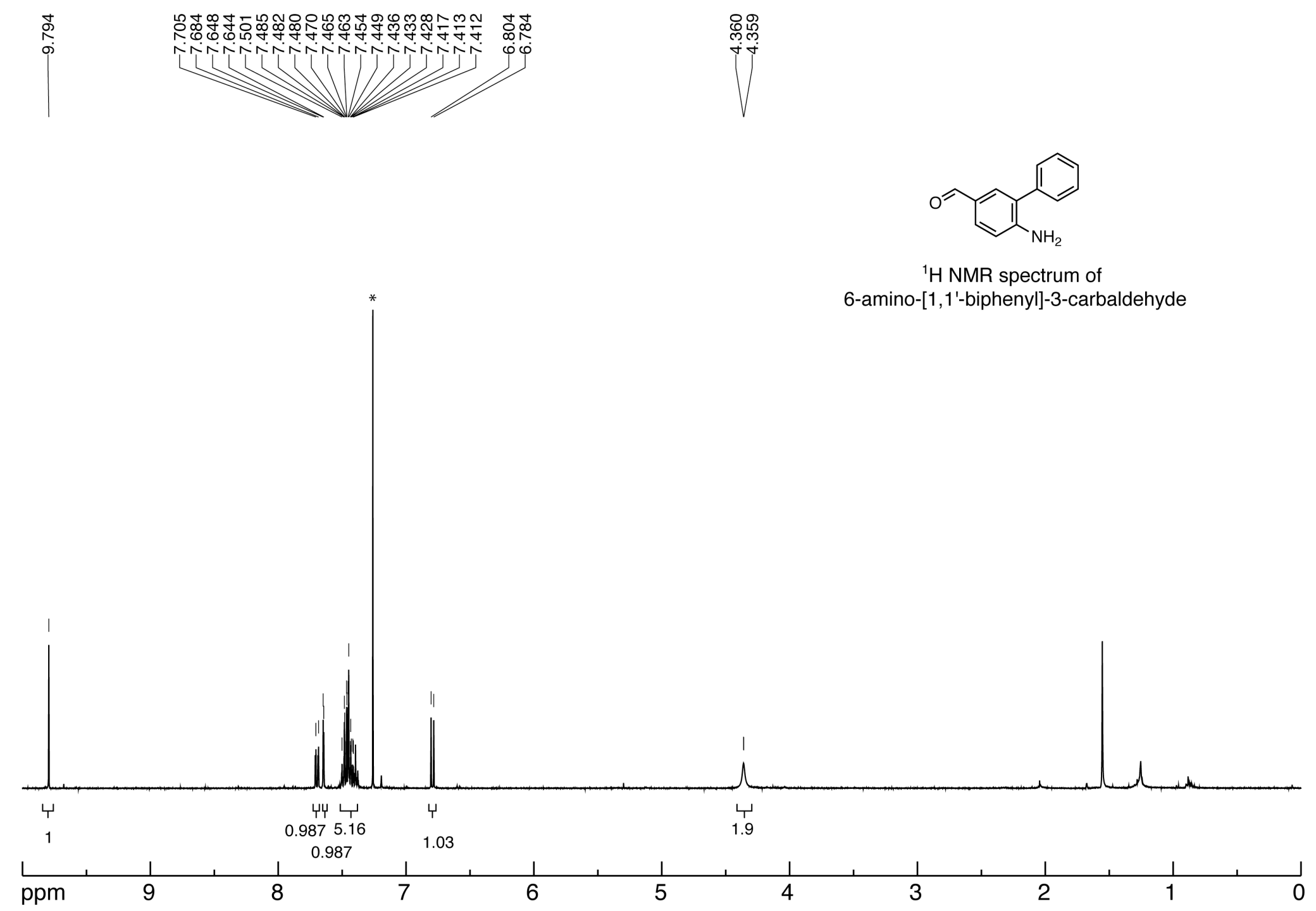

Figure S27. ${ }^{1} \mathrm{H}$ NMR spectrum of 6-amino-[1,1'-biphenyl]-3-carbaldehyde (S1h) in $\mathrm{CDCl}_{3}(400 \mathrm{MHz})$ at $23{ }^{\circ} \mathrm{C}$. $\mathrm{CDCl}_{3}$ solvent peak is marked with *. 


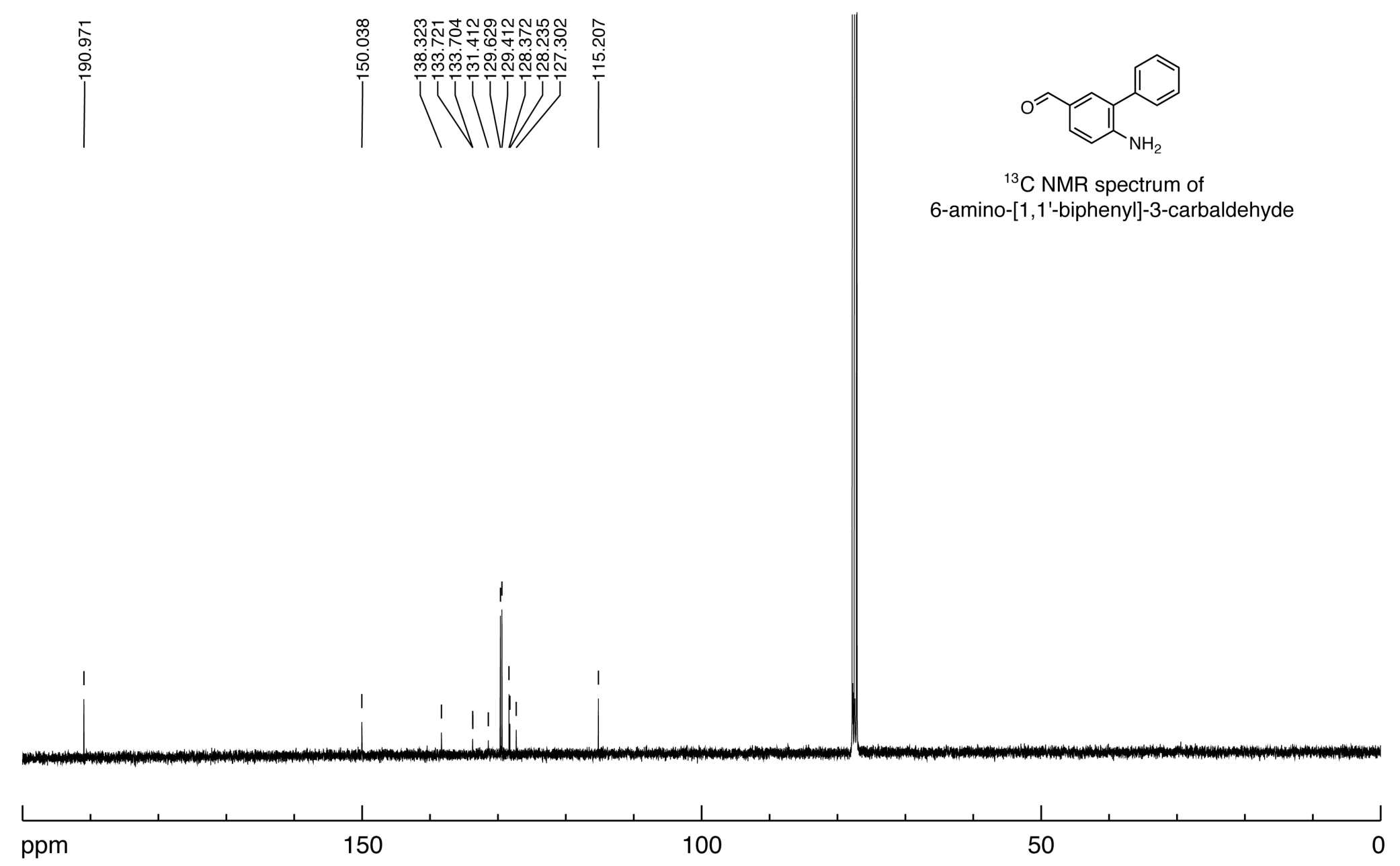

Figure S28. ${ }^{13} \mathrm{C}$ NMR spectrum of 6-amino-[1,1'-biphenyl]-3-carbaldehyde (S1h) in $\mathrm{CDCl}_{3}(100 \mathrm{MHz})$ at $23{ }^{\circ} \mathrm{C} . \mathrm{CDCl}_{3}$ solvent peak is marked with *. 


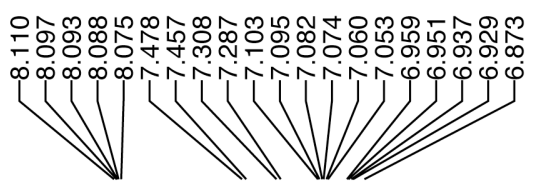

in<smiles>CC(=O)Nc1ccc(F)cc1-c1ccc(Cl)cc1</smiles>

${ }^{1} \mathrm{H}$ NMR spectrum of $N$-(4'-chloro-5-fluoro-[1,1'-biphenyl]-2-yl)acetamide

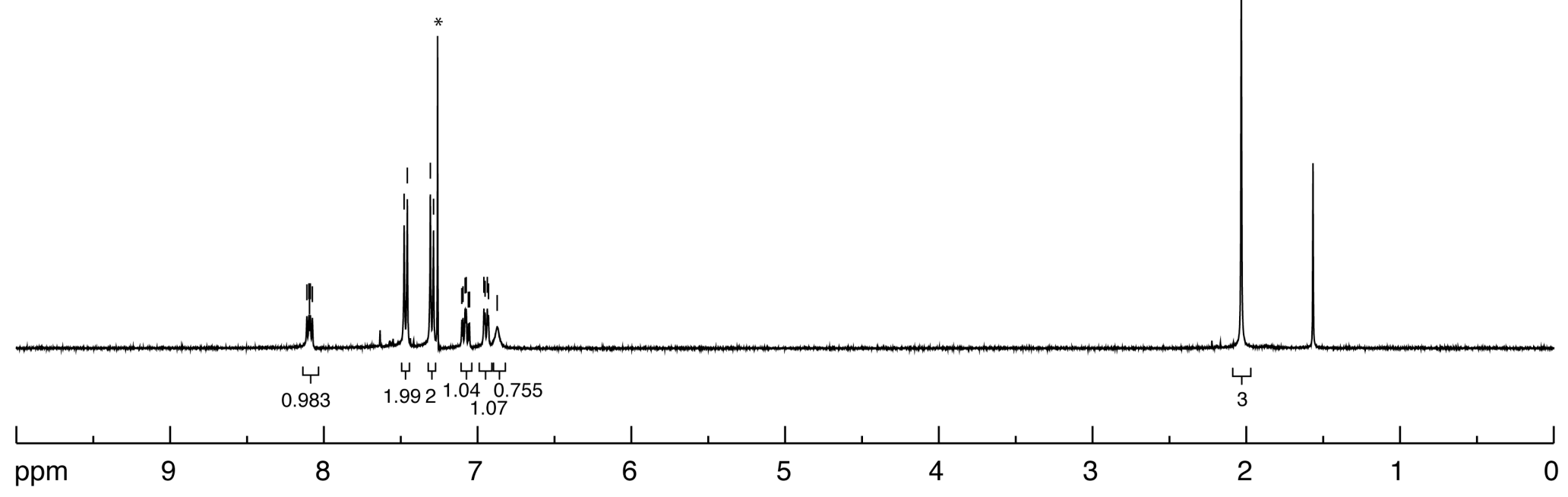

Figure S29. ${ }^{1} \mathrm{H}$ NMR spectrum of $N$-(4'-chloro-5-fluoro-[1,1'-biphenyl]-2-yl)acetamide (au) in $\mathrm{CDCl}_{3}(400 \mathrm{MHz})$ at $23{ }^{\circ} \mathrm{C} . \mathrm{CDCl}_{3}$ solvent peak is marked with *.

S60 


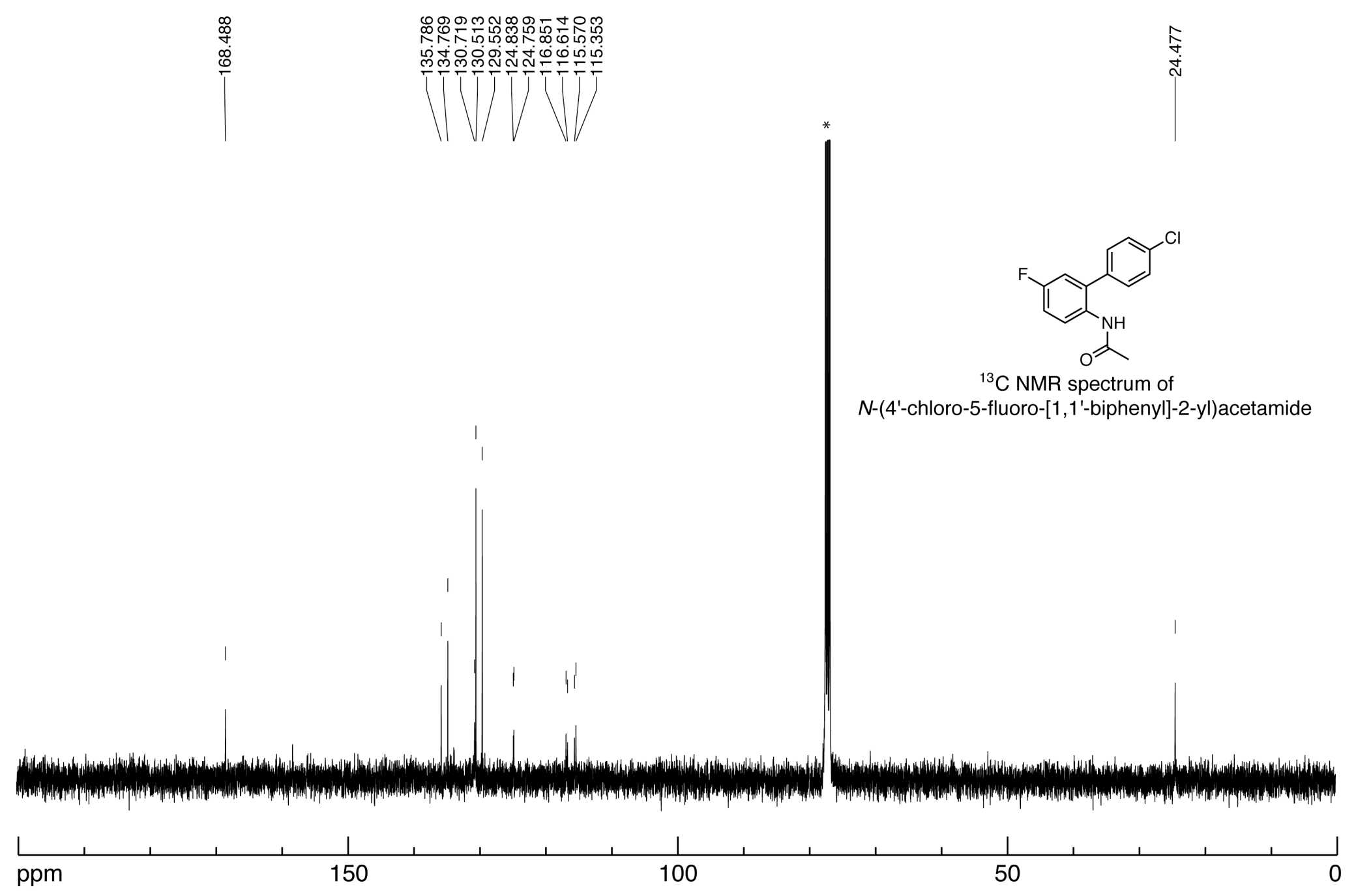

Figure S30. ${ }^{13} \mathrm{C}$ NMR spectrum of $\mathrm{N}$-(4'-chloro-5-fluoro-[1,1'-biphenyl]-2-yl)acetamide (1u) in $\mathrm{CDCl}_{3}\left(100 \mathrm{MHz}\right.$ at $23{ }^{\circ} \mathrm{C} . \mathrm{CDCl}_{3}$ solvent peak is marked with *. 


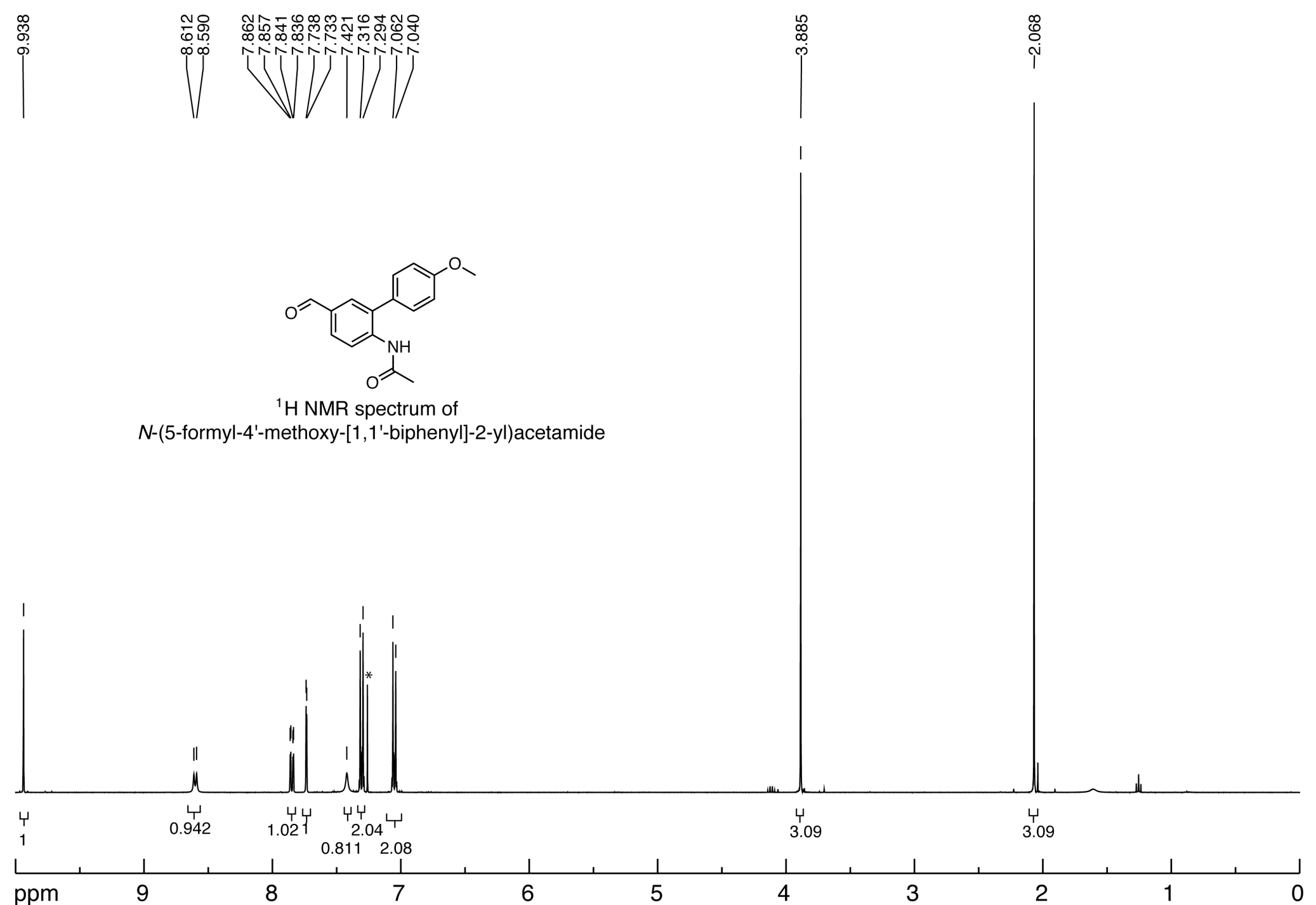

Figure S31. ${ }^{1} \mathrm{H}$ NMR spectrum of $N$-(5-formyl-4'-methoxy-[1,1'-biphenyl]-2-yl)acetamide $(\mathbf{1 v})$ in $\mathrm{CDCl}_{3}\left(400 \mathrm{MHz}\right.$ at $23{ }^{\circ} \mathrm{C}$. $\mathrm{CDCl}_{3}$ solvent peak is marked with *. 

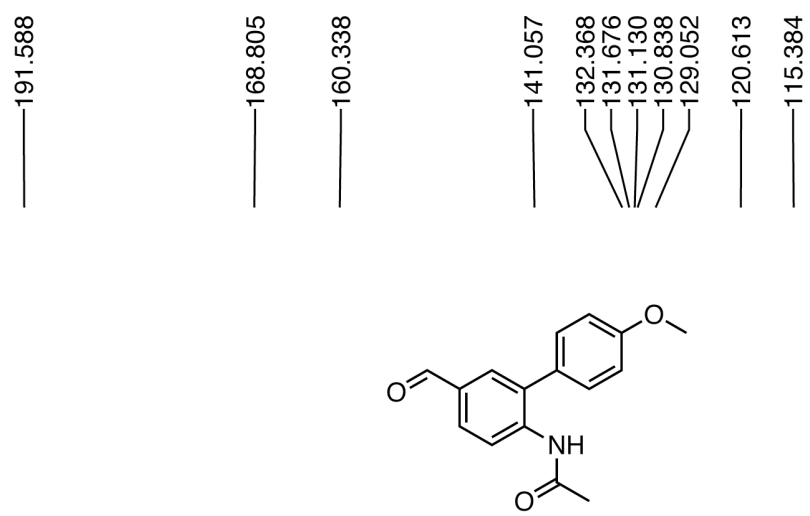

${ }^{13} \mathrm{C}$ NMR spectrum of

$\mathrm{N}$-(5-formyl-4'-methoxy-[1,1'-biphenyl]-2-yl)acetamide

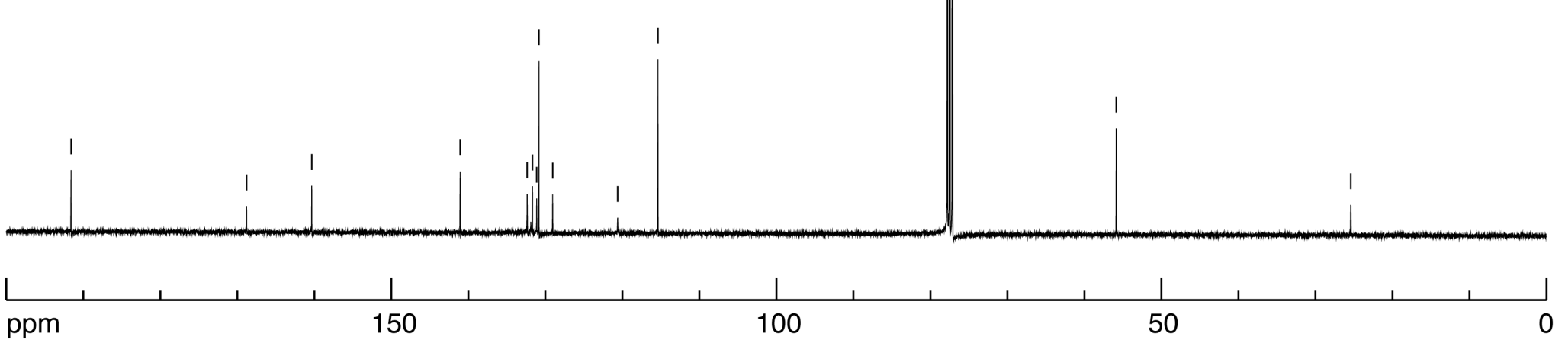

Figure S32. ${ }^{13} \mathrm{C}$ NMR spectrum of $\mathrm{N}$-(5-formyl-4'-methoxy-[1,1'-biphenyl]-2-yl)acetamide (1v) in $\mathrm{CDCl}_{3}(100 \mathrm{MHz})$ at $23{ }^{\circ} \mathrm{C}$. $\mathrm{CDCl}_{3}$ solvent peak is marked with *. 

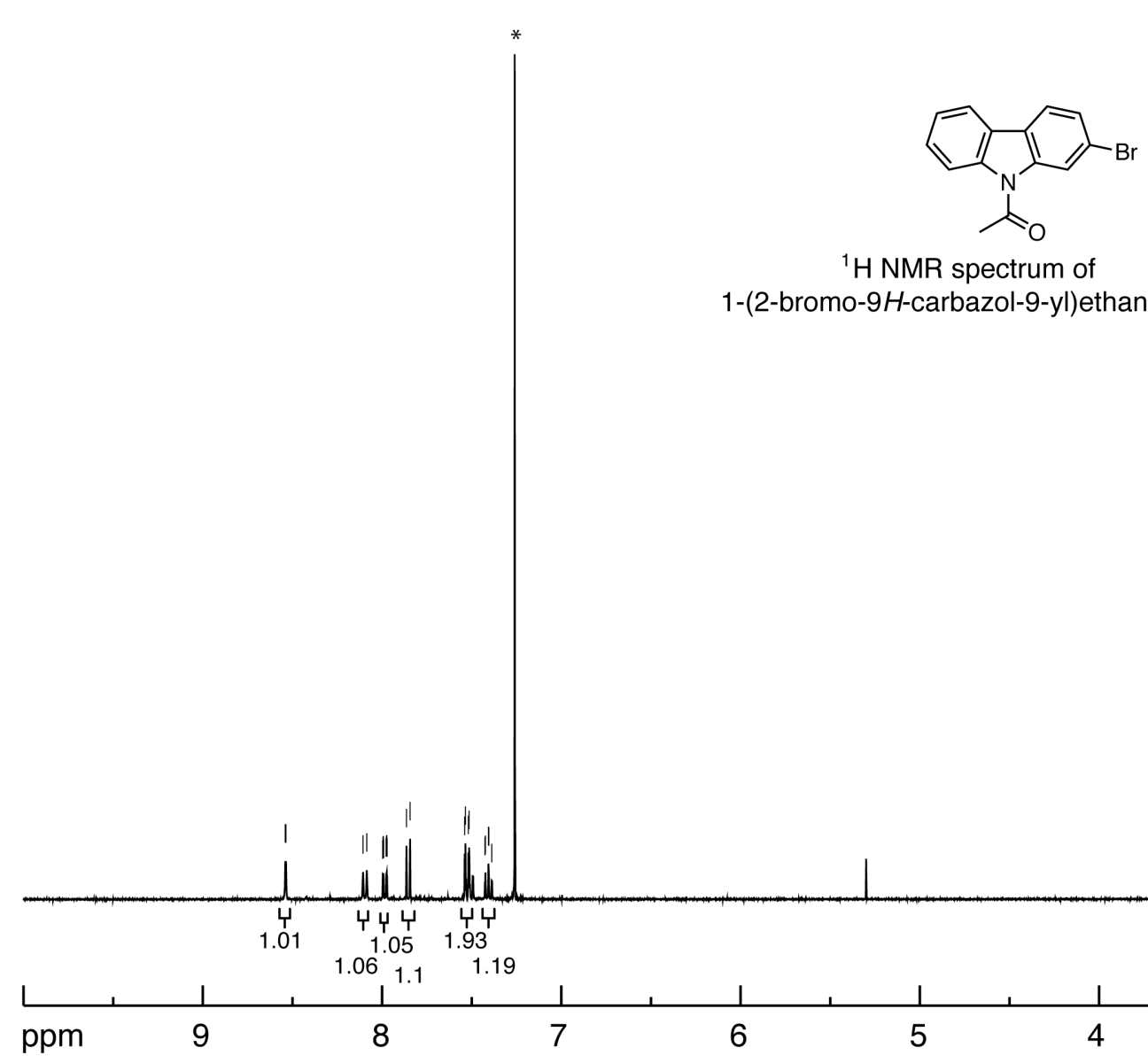

1-(2-bromo-9H-carbazol-9-yl)ethan-1-one

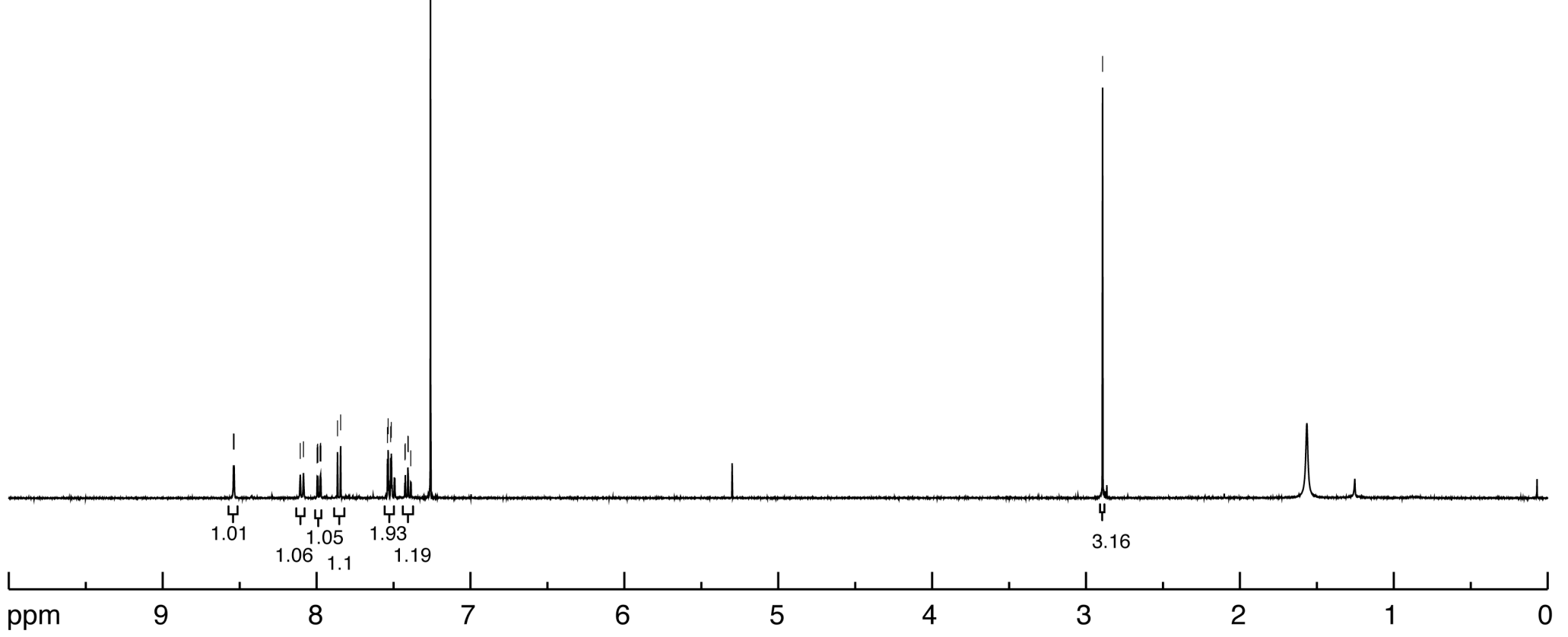

Figure S33. ${ }^{1} \mathrm{H}$ NMR spectrum of 1-(2-bromo-9H-carbazol-9-yl)ethan-1-one (2e) in $\mathrm{CDCl}_{3}(400 \mathrm{MHz})$ at $23{ }^{\circ} \mathrm{C} . \mathrm{CDCl}_{3}$ solvent peak is marked with *. 


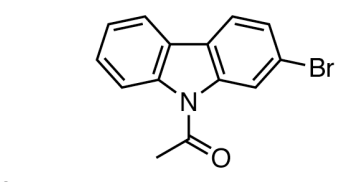

${ }^{13} \mathrm{C}$ NMR spectrum of 1-(2-bromo-9H-carbazol-9-yl)ethan-1-one

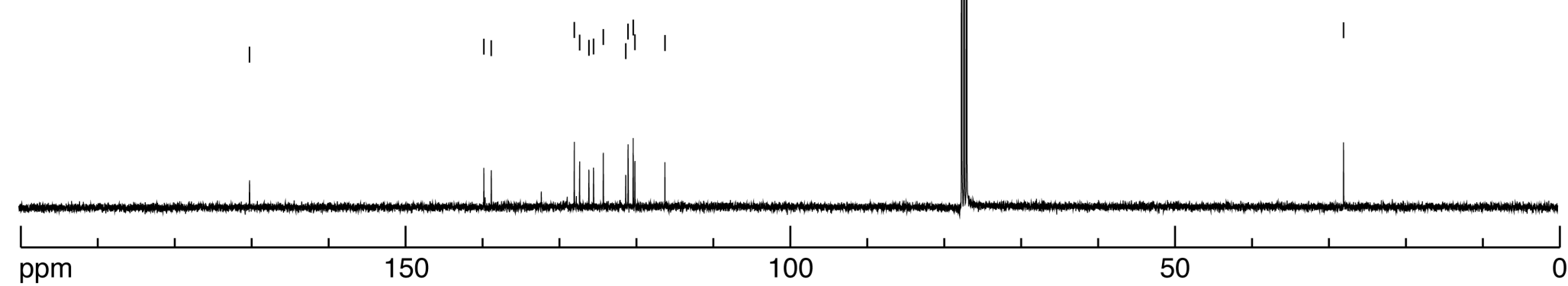

Figure S34. ${ }^{13} \mathrm{C}$ NMR spectrum of 1-(2-bromo-9H-carbazol-9-yl)ethan-1-one (2e) in $\mathrm{CDCl}_{3}\left(100 \mathrm{MHz}^{2}\right.$ at $23{ }^{\circ} \mathrm{C} . \mathrm{CDCl}_{3}$ solvent peak is marked with *. 

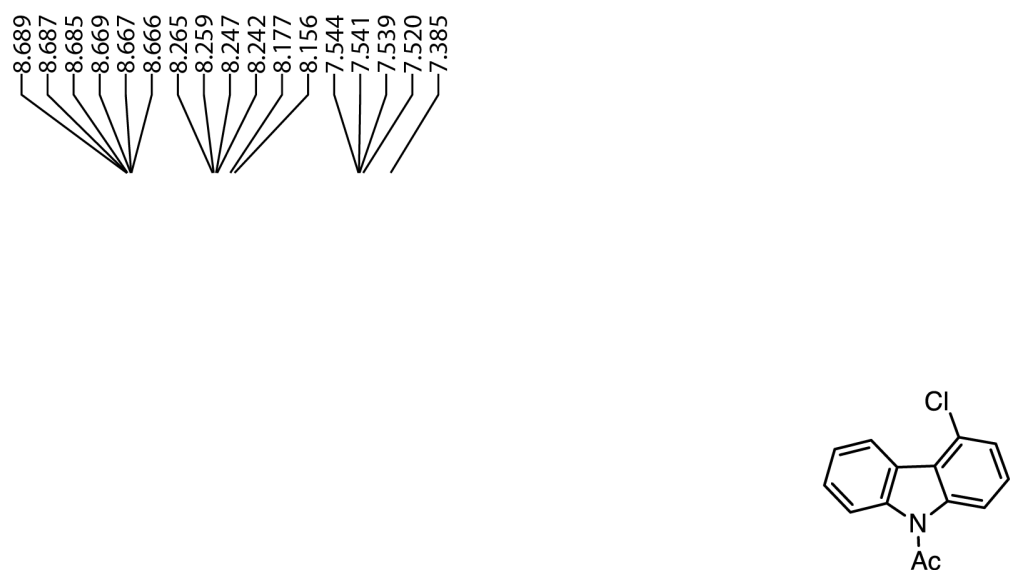

' $\mathrm{H}$ NMR spectrum of

1-(4-chloro-9H-carbazol-9-yl)ethane-1-one

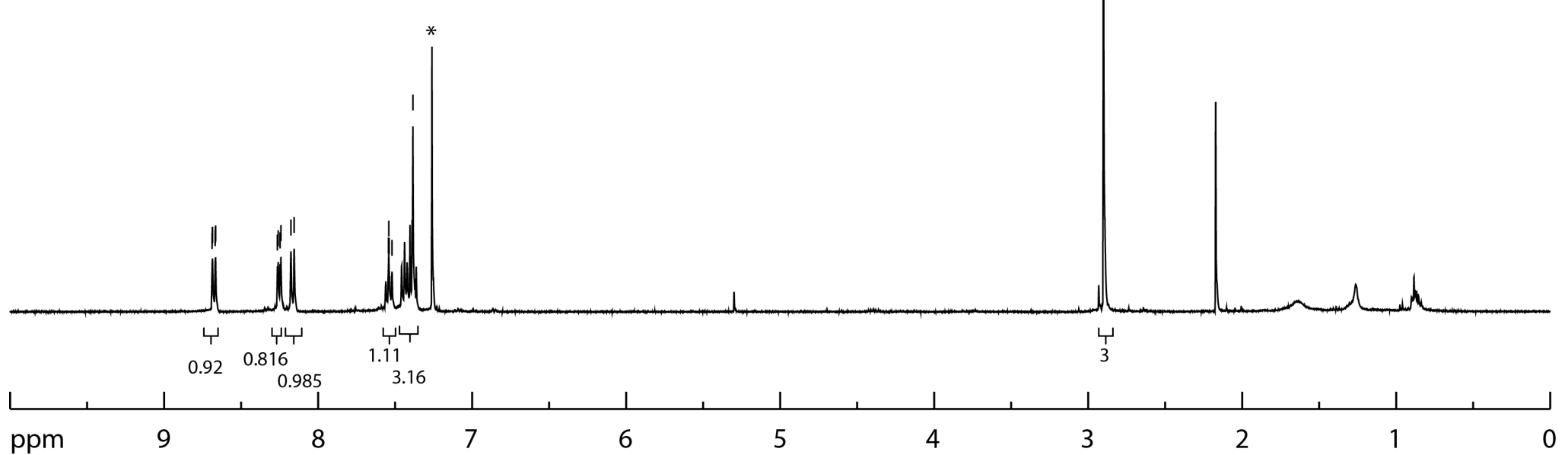

Figure S35. ${ }^{1} \mathrm{H}$ NMR spectrum of 1-(4-chloro-9H-carbazol-9-yl)ethan-1-one (2q) in $\mathrm{CDCl}_{3}\left(400 \mathrm{MHz}^{2}\right.$ at $23{ }^{\circ} \mathrm{C} . \mathrm{CDCl}_{3} \mathrm{Solvent}$ peak is marked with *. 


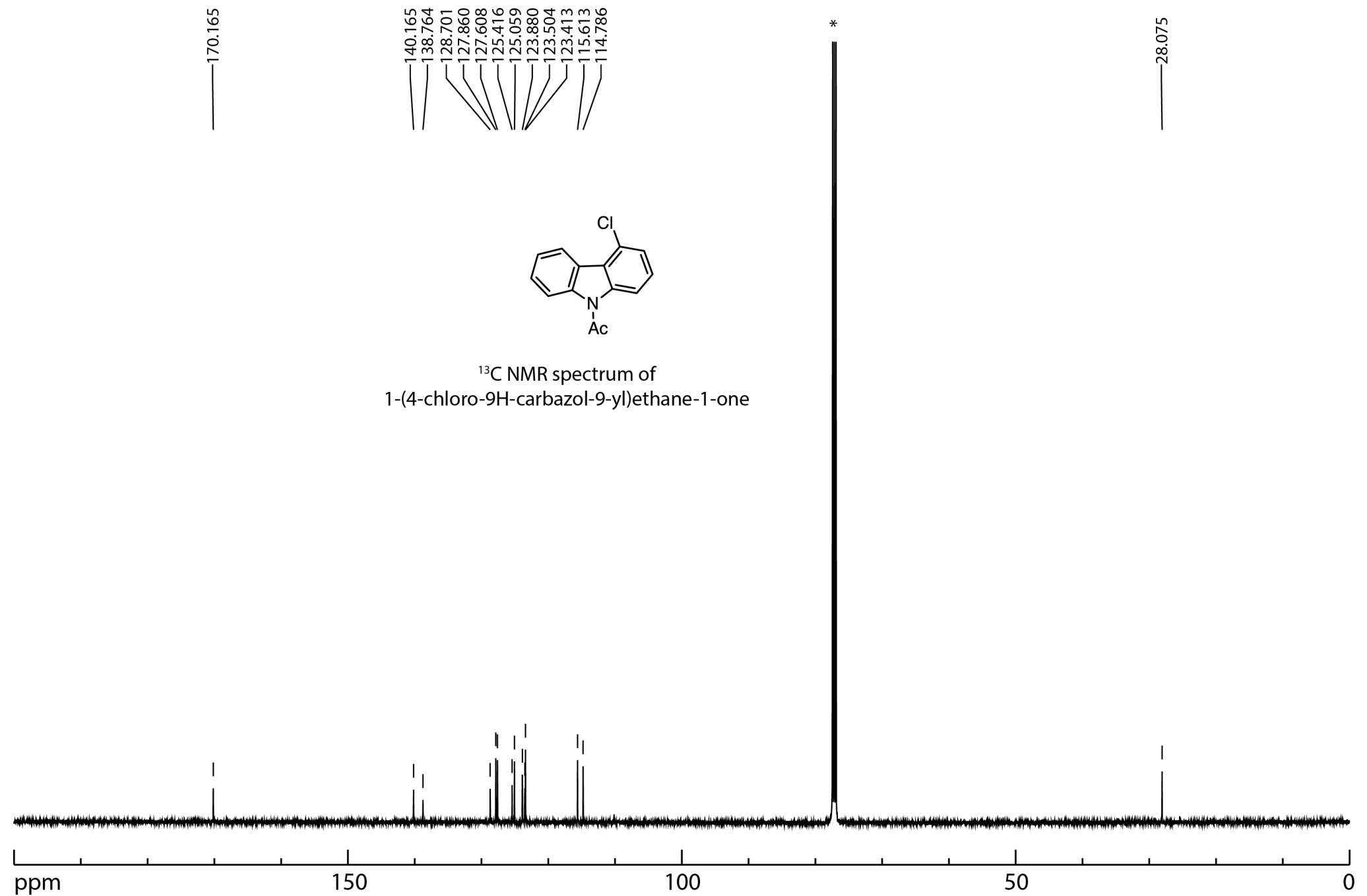

Figure S36. ${ }^{13} \mathrm{C}$ NMR spectrum of 1-(4-chloro-9H-carbazol-9-yl)ethan-1-one (2q) in $\mathrm{CDCl}_{3}(125 \mathrm{MHz})$ at $23{ }^{\circ} \mathrm{C} . \mathrm{CDCl}_{3}$ solvent peak is marked with *. 

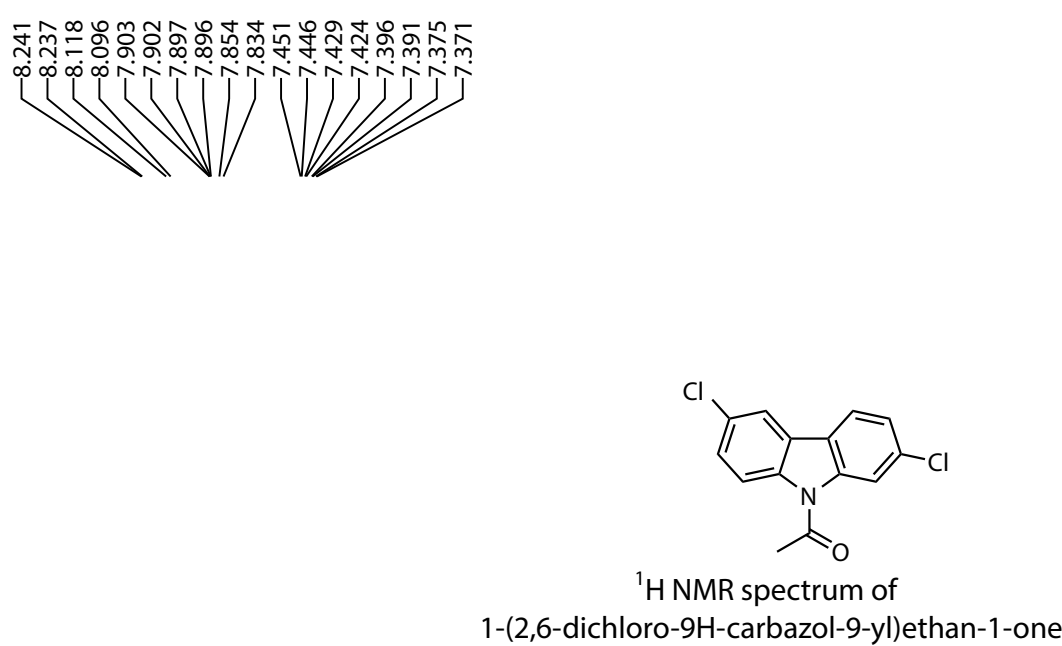

'H NMR spectrum of

1-(2,6-dichloro-9H-carbazol-9-yl)ethan-1-one

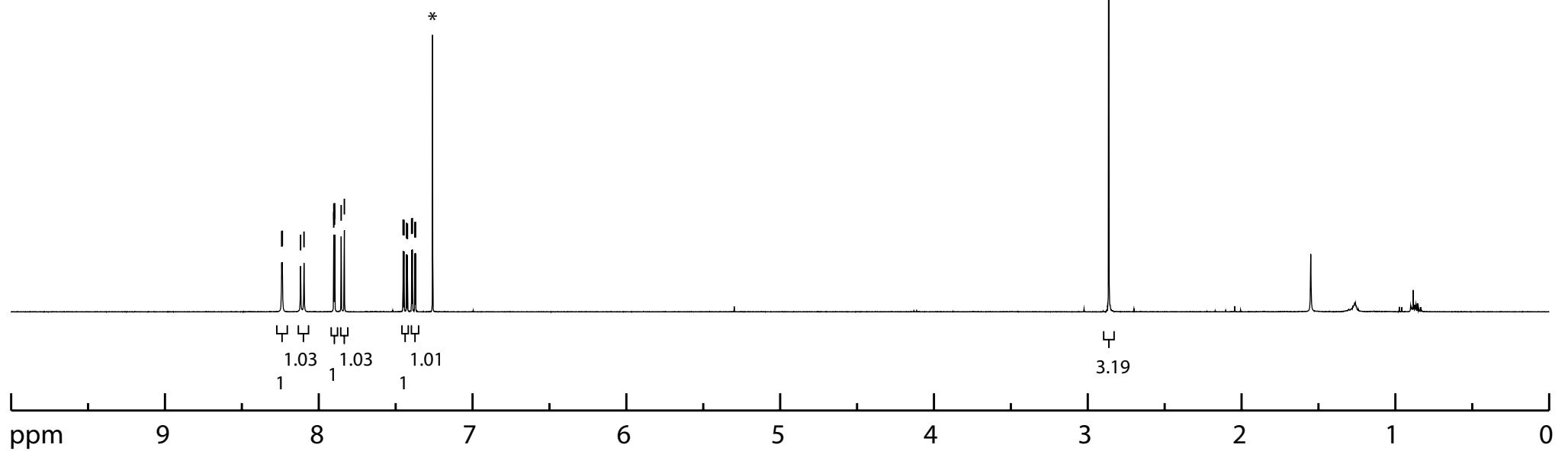

Figure S37. ${ }^{1} \mathrm{H}$ NMR spectrum of 1-(2,6-dichloro-9H-carbazol-9-yl)ethan-1-one (2t) in $\mathrm{CDCl}_{3}(400 \mathrm{MHz})$ at $23{ }^{\circ} \mathrm{C}$. $\mathrm{CDCl}_{3}$ solvent peak is marked with *. 


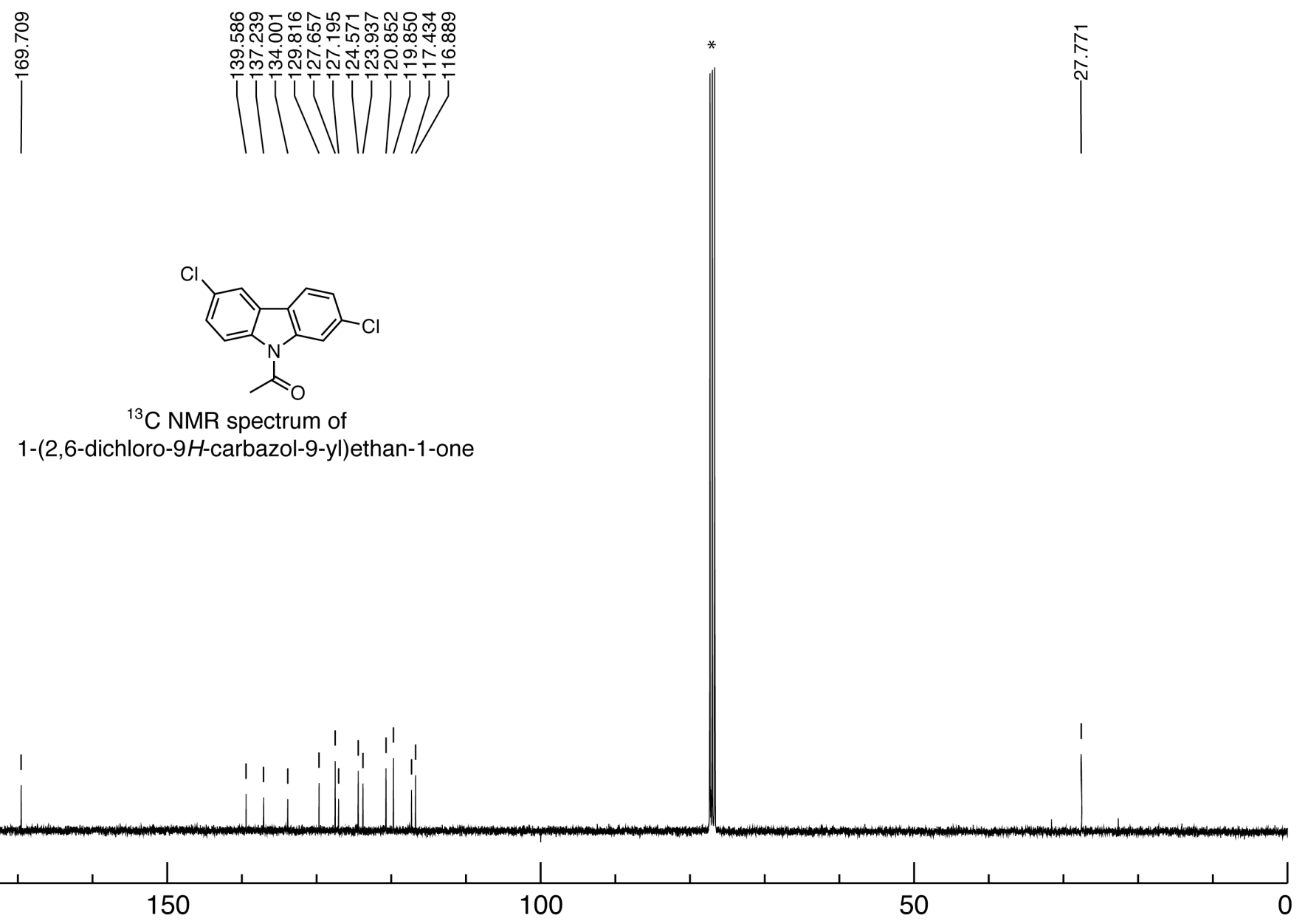

Figure S38. ${ }^{13} \mathrm{C}$ NMR spectrum of 1-(2,6-dichloro-9H-carbazol-9-yl)ethan-1-one (2t) in $\mathrm{CDCl}_{3}(100 \mathrm{MHz})$ at $23{ }^{\circ} \mathrm{C} . \mathrm{CDCl}_{3} \mathrm{solvent}$ peak is marked with *. 

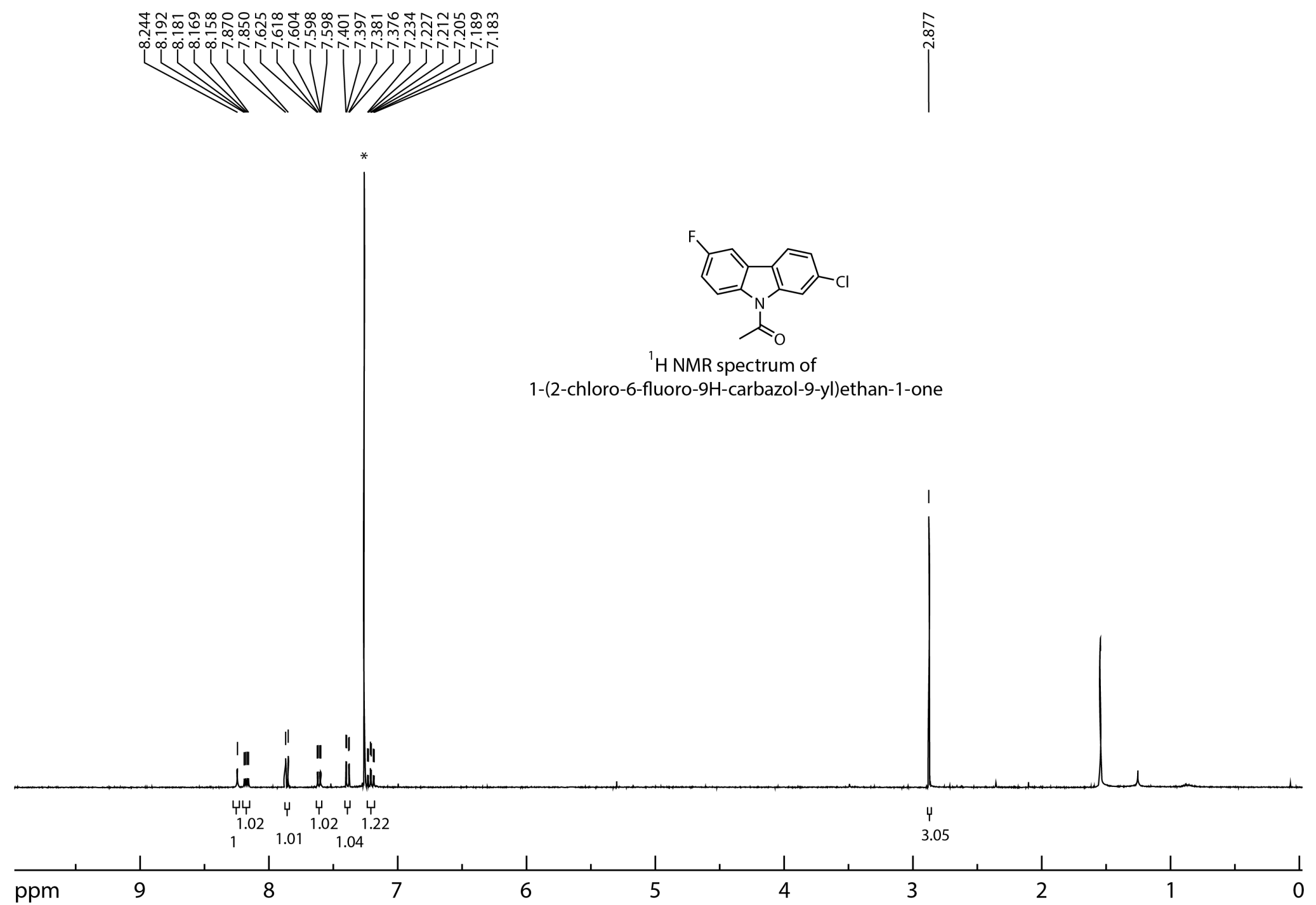

Figure S39. ${ }^{1} \mathrm{H}$ NMR spectrum of 1-(2-chloro-6-fluoro-9H-carbazol-9-yl)ethan-1-one (2u) in $\mathrm{CDCl}_{3}\left(400 \mathrm{MHz}^{2}\right.$ at $23{ }^{\circ} \mathrm{C} . \mathrm{CDCl}_{3}$ solvent peak is marked with *. 


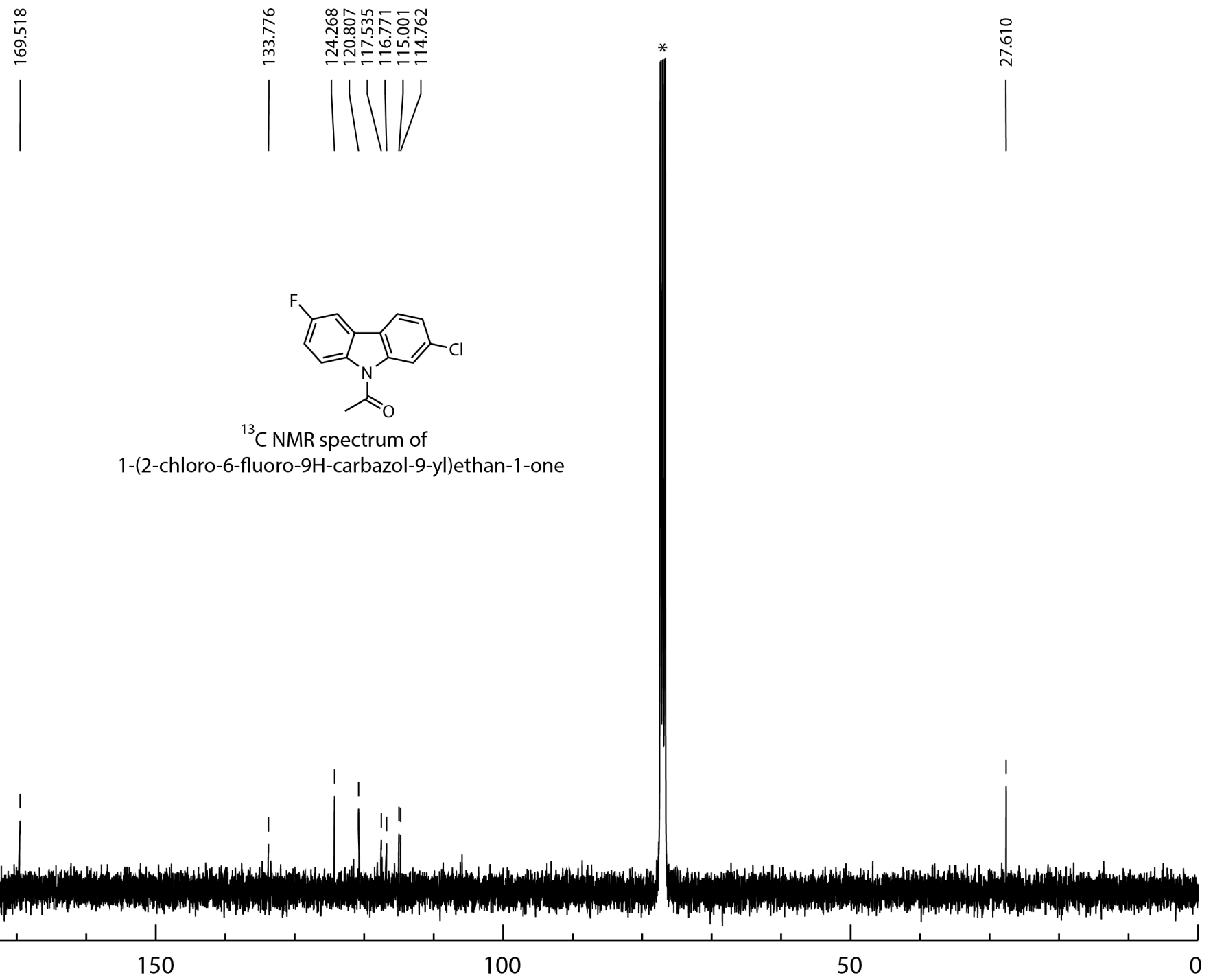

Figure S40. ${ }^{13} \mathrm{C}$ NMR spectrum of 1-(2-chloro-6-fluoro-9H-carbazol-9-yl)ethan-1-one (2u) in $\mathrm{CDCl}_{3}\left(100 \mathrm{MHz}\right.$ at $23{ }^{\circ} \mathrm{C}$. $\mathrm{CDCl} 3$ solvent peak is marked with *. 


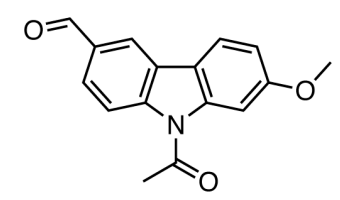

${ }^{1} \mathrm{H}$ NMR spectrum of

9-acetyl-7-methoxy-9H-carbazole-3-carbaldehyde

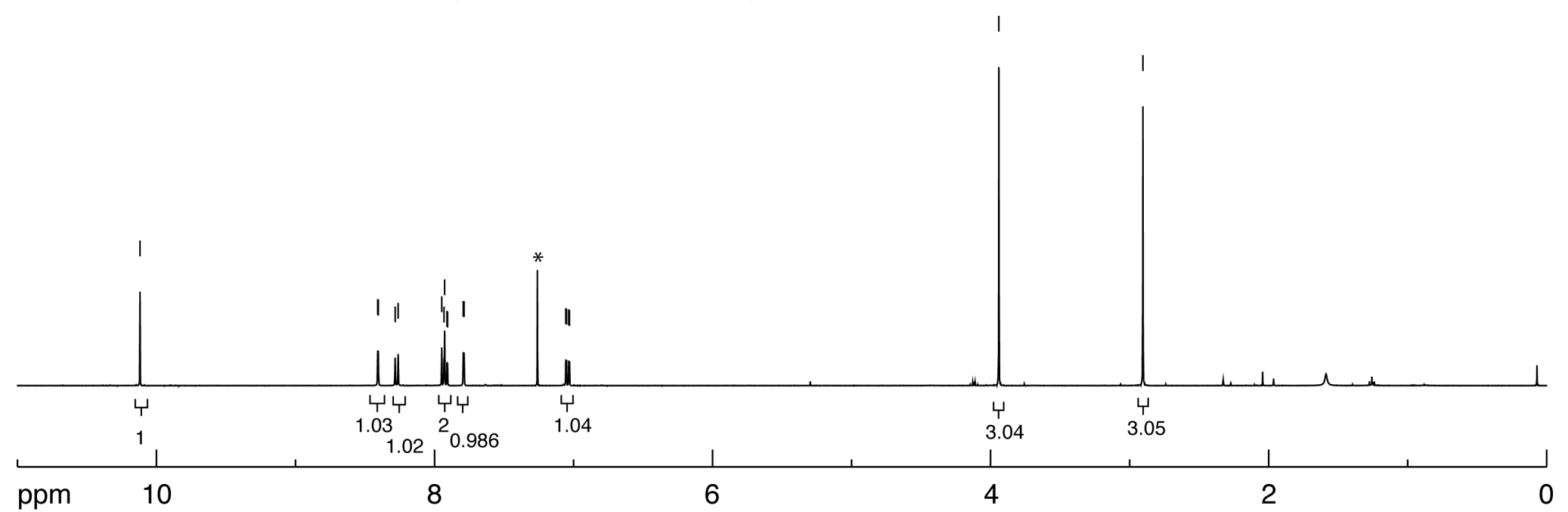

Figure S41. ${ }^{1} \mathrm{H}$ NMR spectrum of 9-acetyl-7-methoxy-9H-carbazole-3-carbaldehyde (2v) in $\mathrm{CDCl}_{3}\left(400 \mathrm{MHz}^{2}\right.$ at $23{ }^{\circ} \mathrm{C} . \mathrm{CDCl}_{3}$ solvent peak is marked with *. 


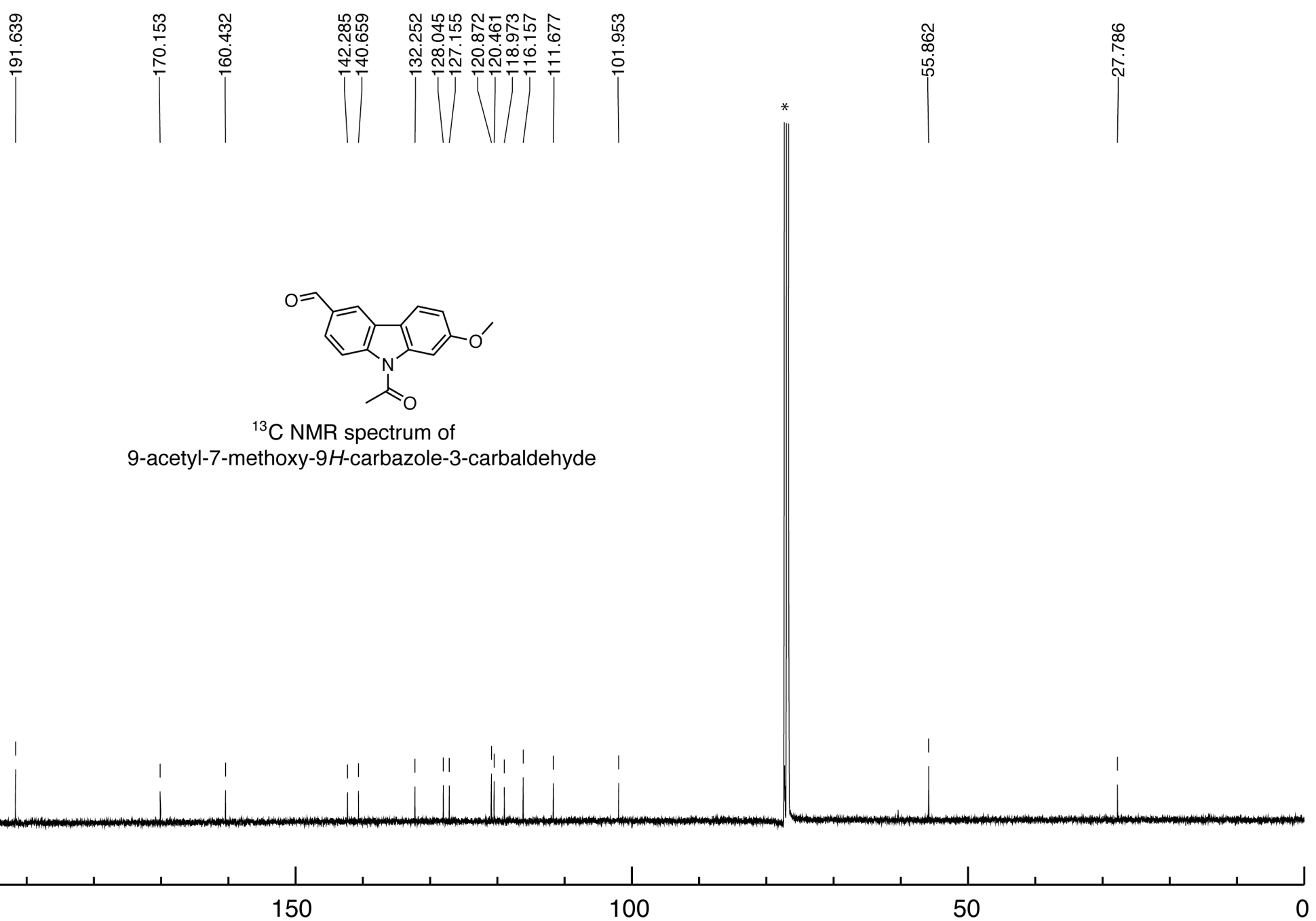

Figure $\mathbf{5 4 2 .}{ }^{13} \mathrm{C}$ NMR spectrum of 9-acetyl-7-methoxy-9H-carbazole-3-carbaldehyde (2v) in $\mathrm{CDCl}_{3}(100 \mathrm{MHz})$ at $23{ }^{\circ} \mathrm{C} . \mathrm{CDCl}_{3}$ solvent peak is marked with *. 

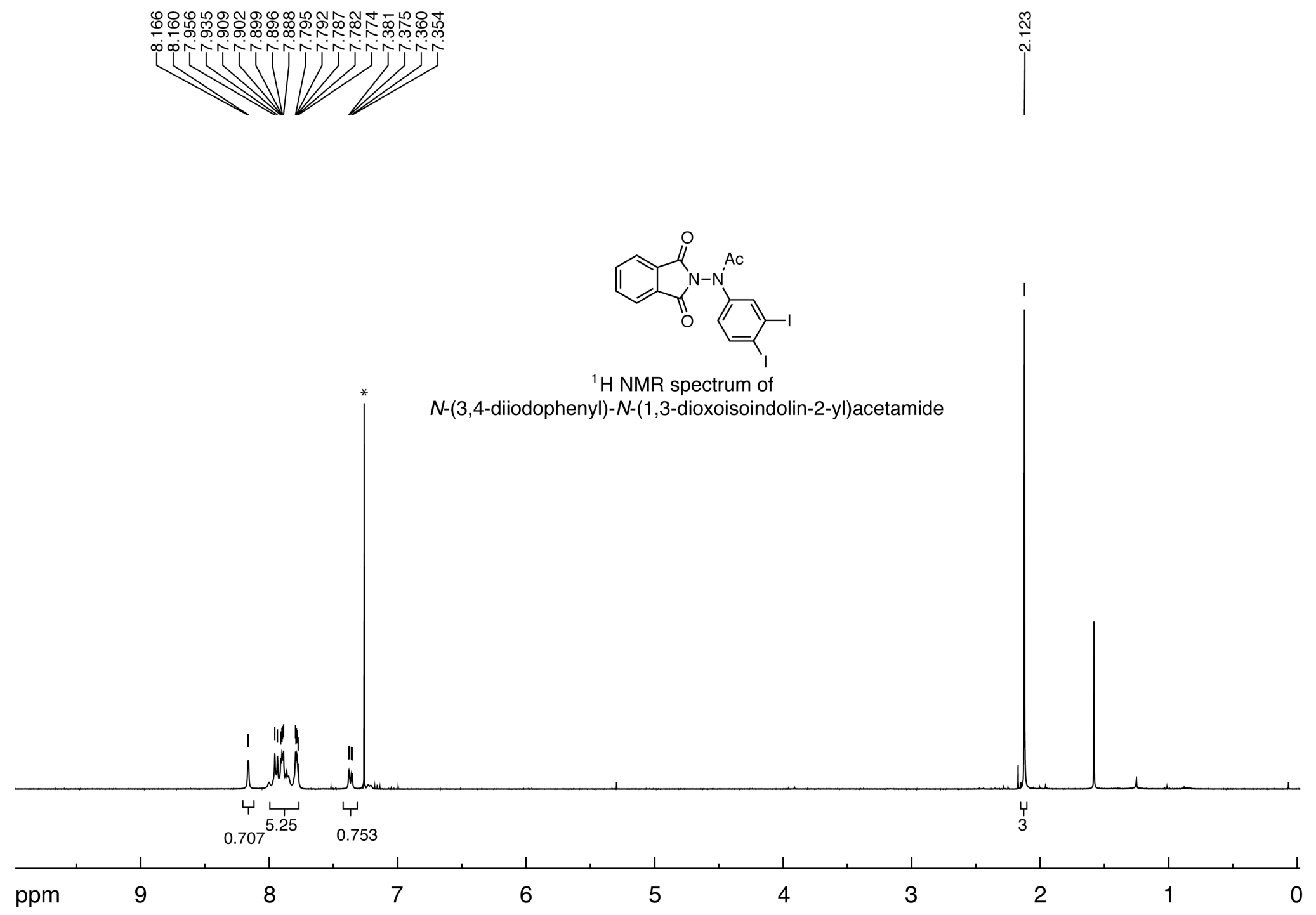

Figure S43. ${ }^{1} \mathrm{H}$ NMR spectrum of $N$-(3,4-diiodophenyl)- $N$-(1,3-dioxoisoindolin-2-yl)acetamide $(\mathbf{5 f})$ in $\mathrm{CDCl} 3{ }_{3}(400 \mathrm{MHz})$ at $23{ }^{\circ} \mathrm{C}$. $\mathrm{CDCl}_{3}$ solvent peak is marked with *. 

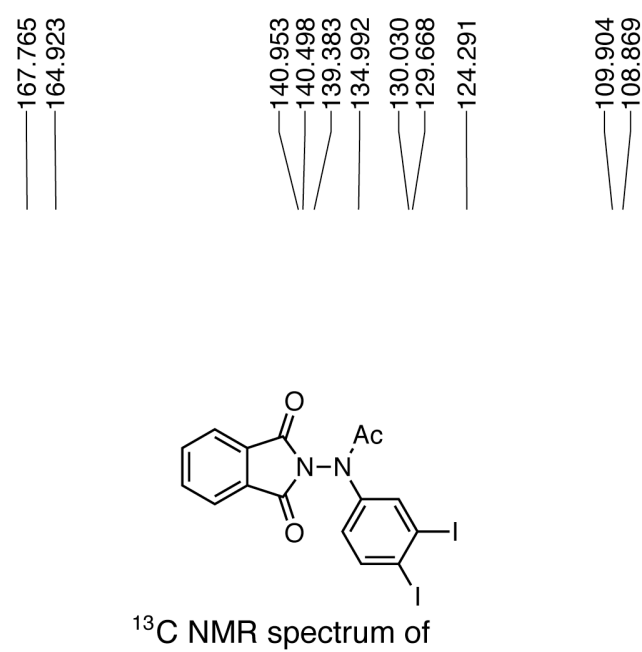

$N$-(3,4-diiodophenyl)- $N$-(1,3-dioxoisoindolin-2-yl)acetamide

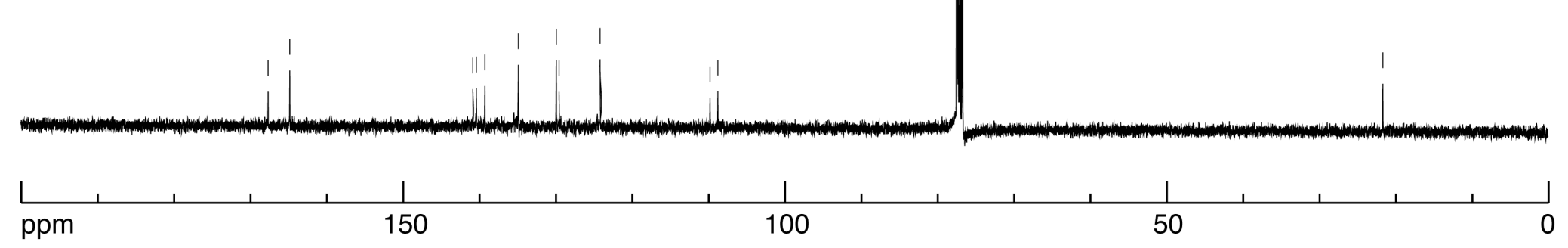

Figure S44. ${ }^{13} \mathrm{C}$ NMR spectrum of $N$-(3,4-diiodophenyl)- $N$-(1,3-dioxoisoindolin-2-yl)acetamide (5f) in $\mathrm{CDCl}_{3}(100 \mathrm{MHz})$ at 23 ${ }^{\circ} \mathrm{C} . \mathrm{CDCl}_{3}$ solvent peak is marked with *. 


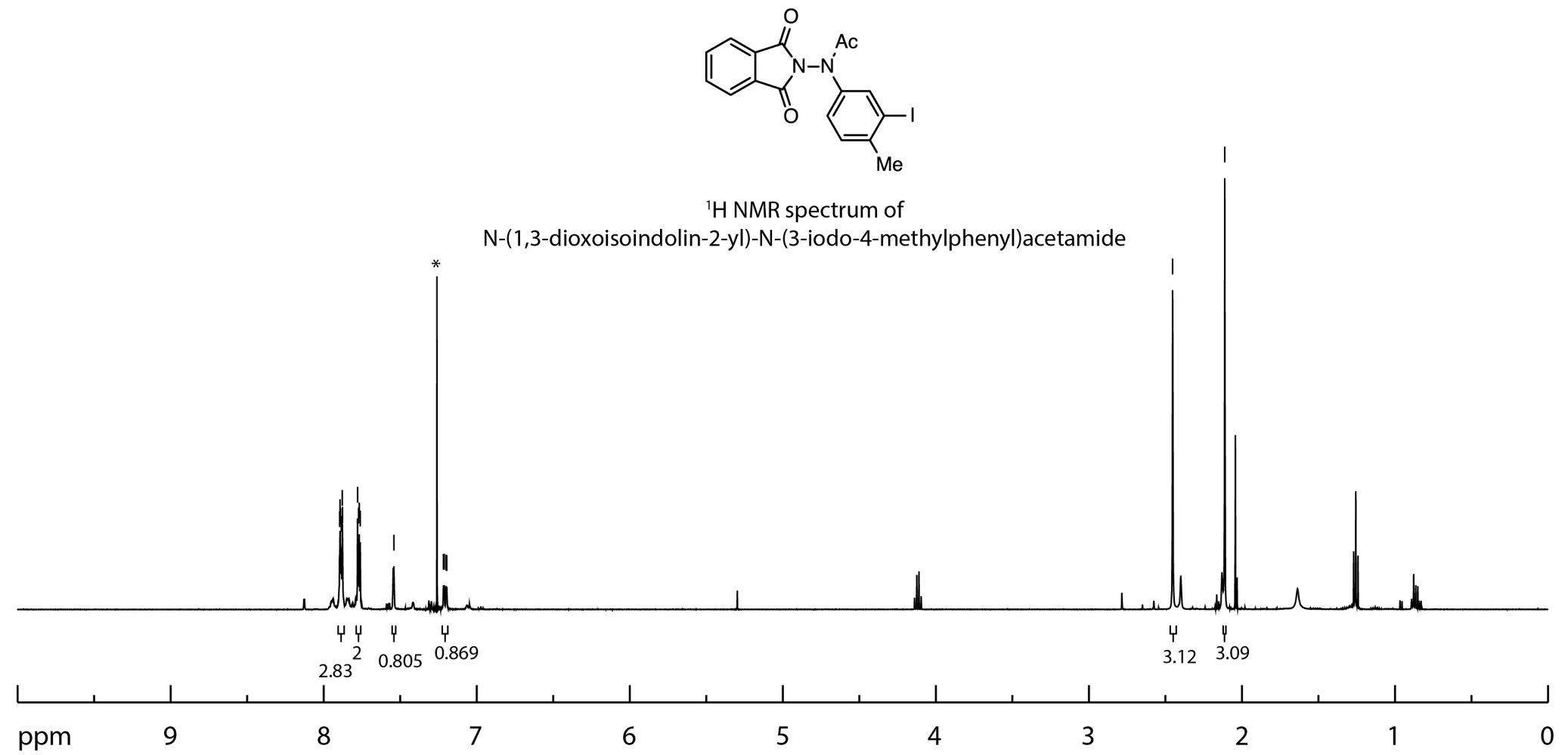

Figure S45. ${ }^{1} \mathrm{H}$ NMR spectrum of $N$-(1,3-dioxoisoindolin-2-yl)- $N$-(3-iodo-4-methylphenyl)acetamide $(5 \mathrm{~g}) \mathrm{in} \mathrm{CDCl}_{3}(500 \mathrm{MHz})$ at $23{ }^{\circ} \mathrm{C} . \mathrm{CDCl}_{3}$ solvent peak is marked with *. 


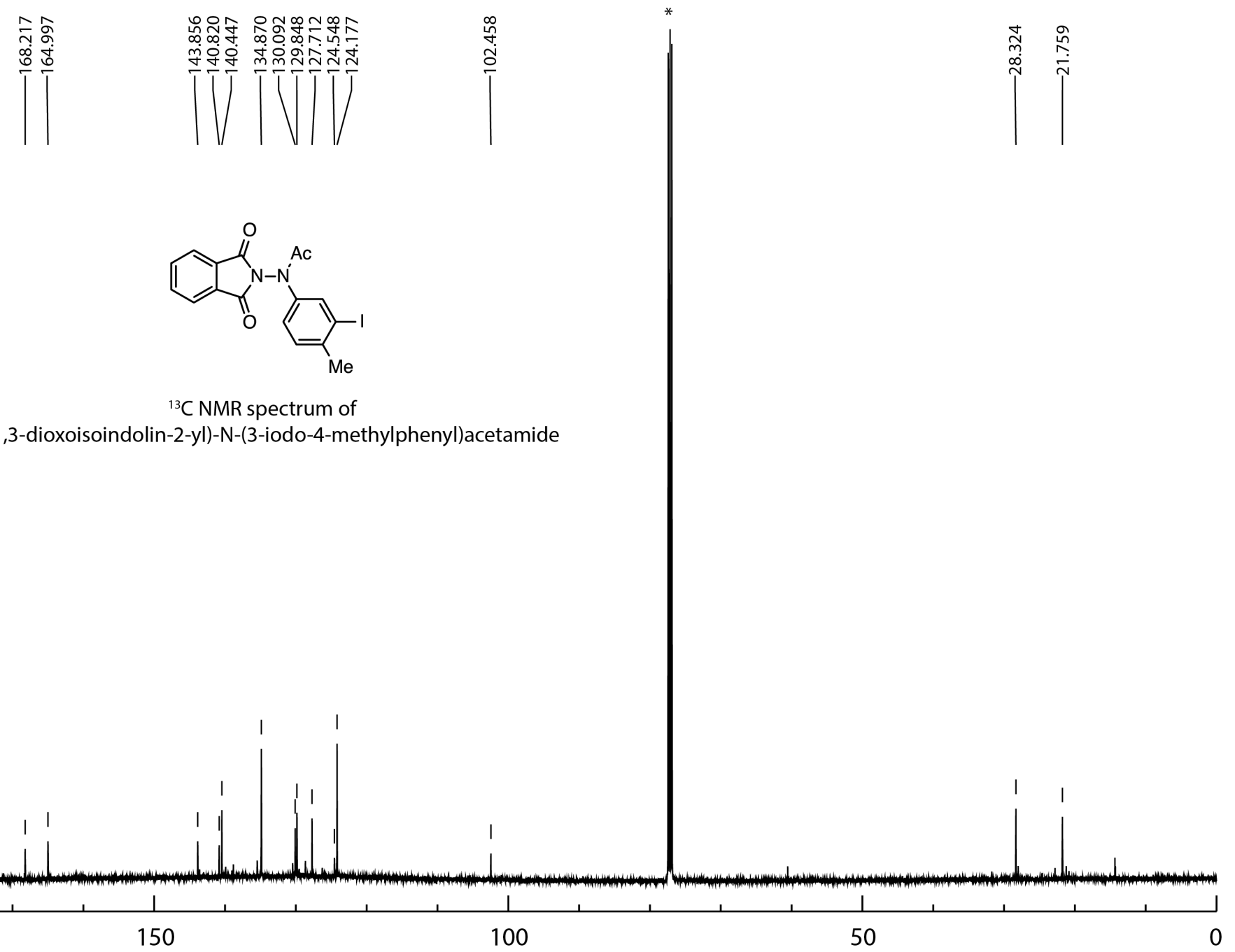

ppm

150

100

50

Figure S46. ${ }^{13} \mathrm{C}$ NMR spectrum of $N$-(1,3-dioxoisoindolin-2-yl)- $N$-(3-iodo-4-methylphenyl)acetamide (5g) in $\mathrm{CDCl}_{3}(125 \mathrm{MHz}$ at $23{ }^{\circ} \mathrm{C} . \mathrm{CDCl}_{3}$ solvent peak is marked with *. 

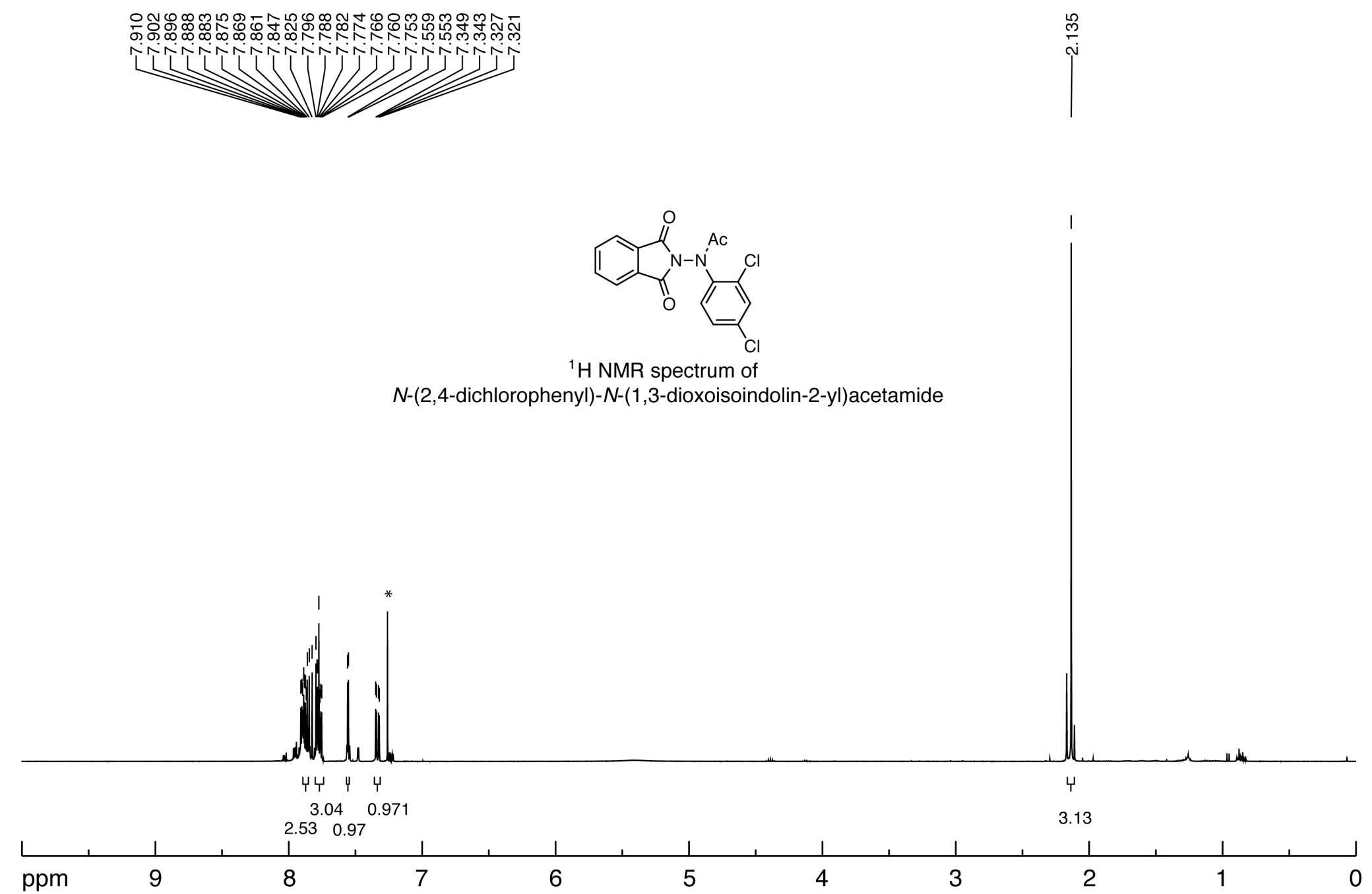

Figure S47. ${ }^{1} \mathrm{H}$ NMR spectrum of $N$-(2,4-dichlorophenyl)-N-(1,3-dioxoisoindolin-2-yl)acetamide (5h) in $\mathrm{CDCl}_{3}(400 \mathrm{MHz})$ at 23 ${ }^{\circ} \mathrm{C} . \mathrm{CDCl}_{3}$ solvent peak is marked with * 


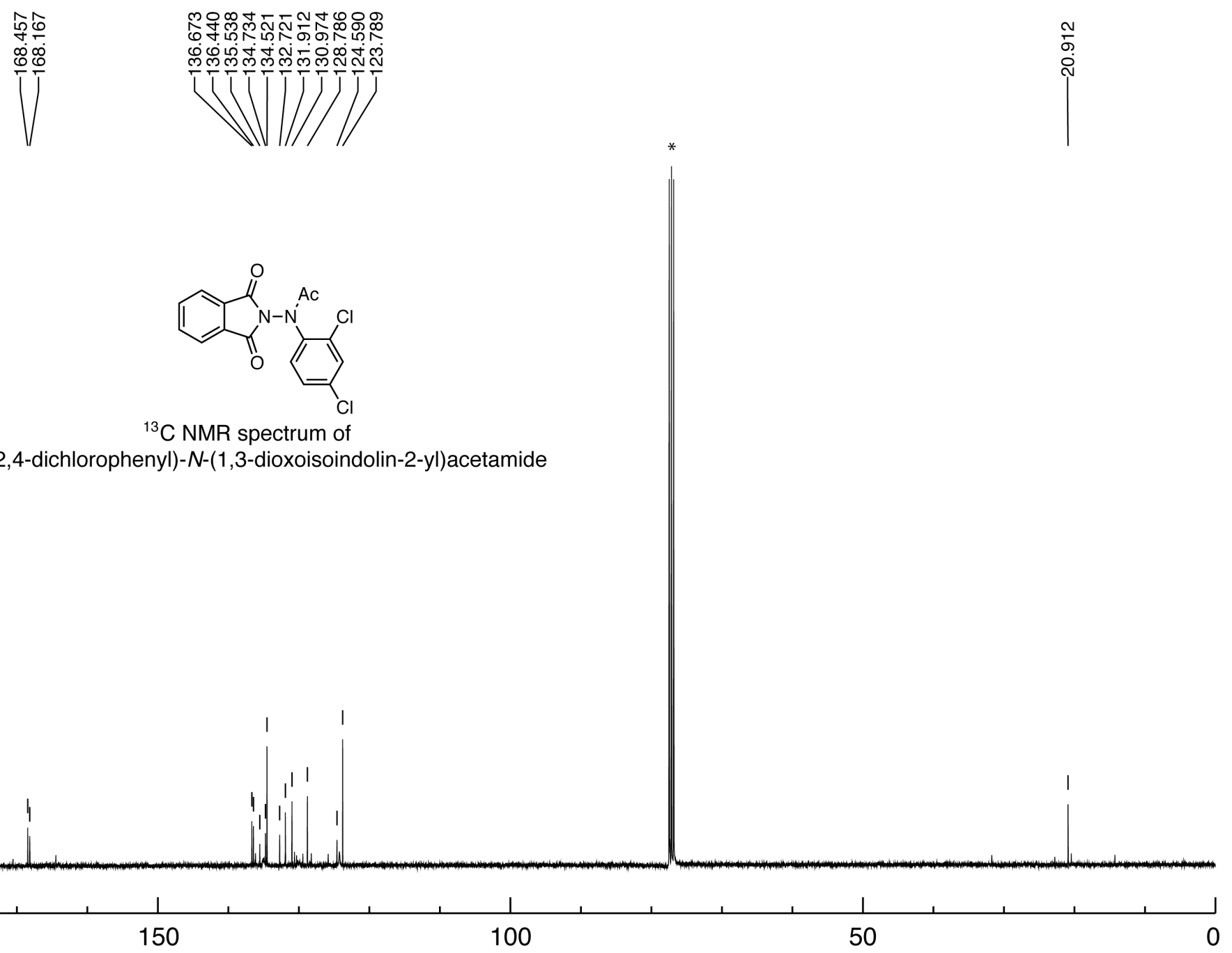

ppm

Figure S48. ${ }^{13} \mathrm{C}$ NMR spectrum of N-(2,4-dichlorophenyl)-N-(1,3-dioxoisoindolin-2-yl)acetamide (5h) in $\mathrm{CDCl}_{3}$ (100 $\left.\mathrm{MHz}\right)$ at 23 ${ }^{\circ} \mathrm{C} . \mathrm{CDCl}_{3}$ solvent peak is marked with *. 

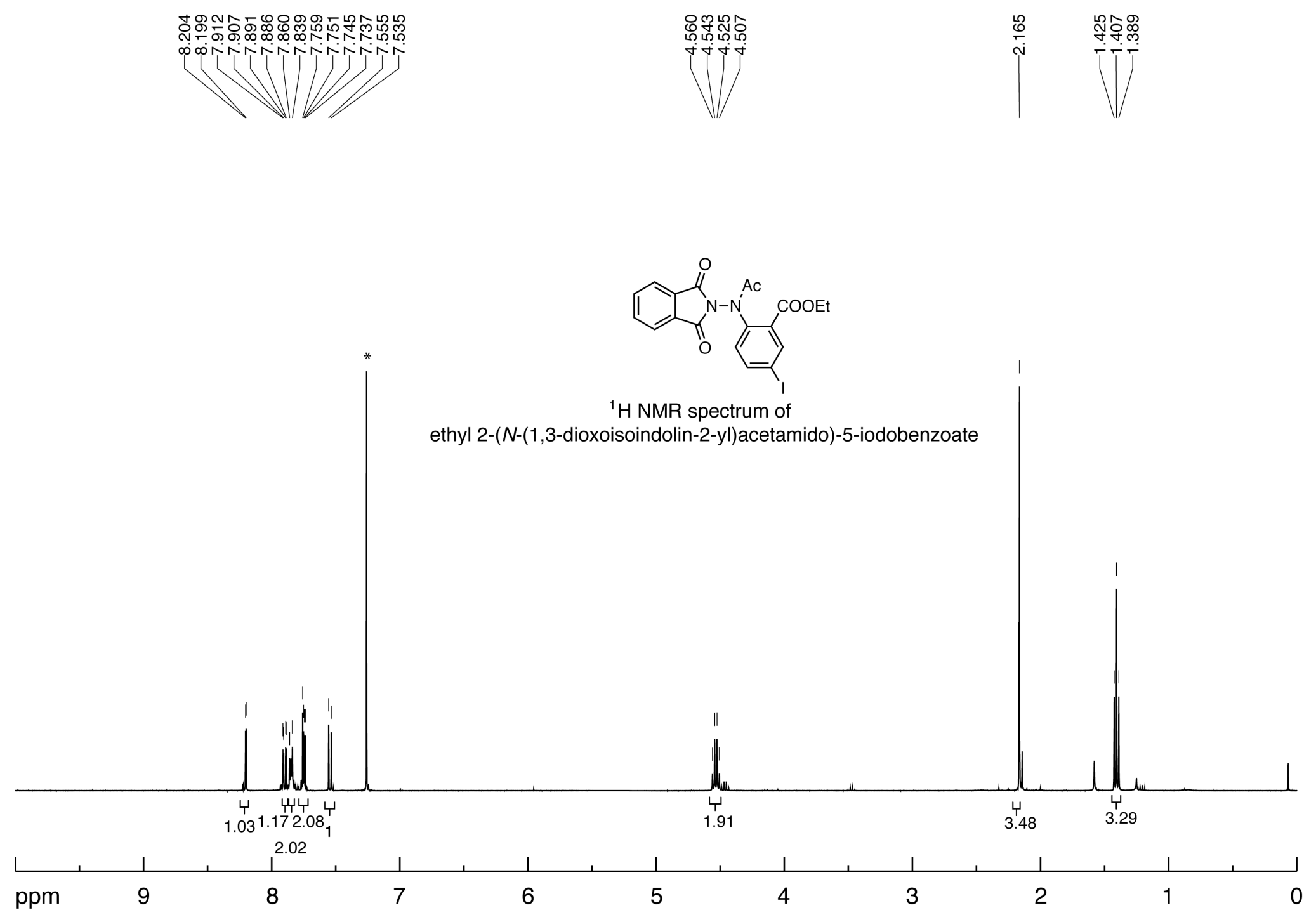

Figure S49. ${ }^{1} \mathrm{H}$ NMR spectrum of ethyl 2-(N-(1,3-dioxoisoindolin-2-yl)acetamido)-5-iodobenzoate (5i) in $\mathrm{CDCl}_{3}$ (400 $\left.\mathrm{MHz}\right)$ at $23{ }^{\circ} \mathrm{C} . \mathrm{CDCl}_{3}$ solvent peak is marked with *. 


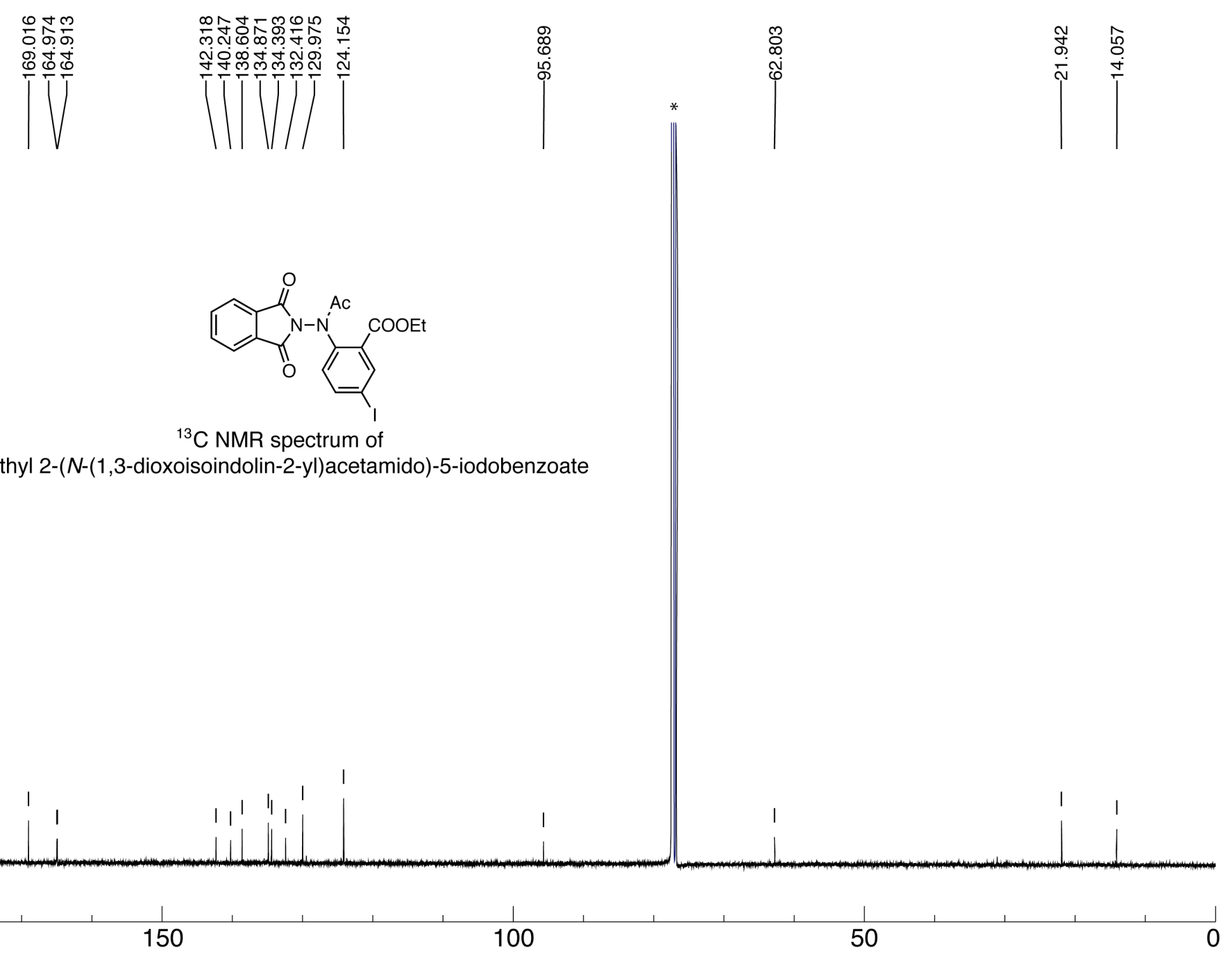

Figure S50. ${ }^{13} \mathrm{C}$ NMR spectrum of ethyl 2-(N-(1,3-dioxoisoindolin-2-yl)acetamido)-5-iodobenzoate (5i) in $\mathrm{CDCl}_{3}(100 \mathrm{MHz})$ at $23{ }^{\circ} \mathrm{C} . \mathrm{CDCl}_{3}$ solvent peak is marked with *. 


\section{F. References:}

1. Powers, D. C.; Lee, E.; Ariafard, A.; Sanford, M. S.; Yates, B. F.; Canty, A. J.; Ritter, T. Connecting Binuclear Pd(III) and Mononuclear Pd(IV) Chemistry by Pd-Pd Bond Cleavage. J. Am. Chem. Soc. 2012, 134, 12002-12009.

2. Dohi, T.; Sasa, H.; Dochi, M.; Yasui, C.; Kita, Y. Oxidative Coupling of N-Methoxyamides and Related Compounds toward Aromatic Hydrocarbons by Designer $\mu$-Oxo Hypervalent Iodine Catalyst. Synthesis 2019, 51, 1185-1195.

3. Mörsdorf, J.-M.; Wadepohl, H.; Ballmann, J. A Tautomeric $\lambda^{3} / \lambda^{5}$-Phosphane Pair and Its Ambiphilic Reactivity. Inorg. chem. 2019, 58, 3502-3508.

4. Brosse, N.; Pinto, M. F.; Jamart-Gregoire, B. Preparation of Multiply Protected Alkylhydrazine Derivatives by Mitsunobu and PTC Approaches. Eur. J. Org. Chem. 2003, 2003, 4757-4764.

5. Liang, Z.; Ju, L.; Xie, Y.; Huang, L.; Zhang, Y. Free-Amine-Directed Alkenylation of C ( $\left.\operatorname{sp}_{2}\right)-$ $\mathrm{H}$ and Cycloamination by Palladium Catalysis. Chem. Eur. J. 2012, 18, 15816-15821.

6. Singh, D. K.; Kim, I. Convergent synthesis of Diptoindonesin G. Tetrahedron Lett. 2019, 60, 300-301.

7. Huq, M. M.; Rahman, M. R.; Naher, M.; Khan, M. M. R.; Masud, M. K.; Hossain, G. M. G.; Zhu, N.; Lo, Y. H.; Younus, M.; Wong, W.-Y. Synthesis, Characterization and Catalytic Activities of Palladium(II) Nitroaryl Complexes. J. Inorg. Organomet. Polym. Mater. 2016, 26, 1243-1252.

8. Fulmer, G. R.; Miller, A. J.; Sherden, N. H.; Gottlieb, H. E.; Nudelman, A.; Stoltz, B. M.; Bercaw, J. E.; Goldberg, K. I. NMR Chemical Shifts of Trace Impurities: Common Laboratory Solvents, Organics, and Gases in Deuterated Solvents Relevant to the Organometallic Chemist. Organometallics 2010, 29, 2176-2179.

9. Wang, W.; Guo, Y.; Sun, K.; Wang, S.; Zhang, S.; Liu, C.; Chen, Q.-Y. Visible Light-Induced Radical Cyclization of Tertiary Bromides with Isonitriles To Construct Trifluoromethylated Quaternary Carbon Center. J. Org. Chem. 2018, 83, 14588-14599.

10. Chatterjee, T.; Lee, D. S.; Cho, E. J. Extended Study of Visible-Light-Induced Photocatalytic [4+ 2] Benzannulation: Synthesis of Polycyclic (Hetero) Aromatics. J. Org. Chem. 2017, 82, 4369-4378.

11. Zuo, Z.; Liu, J.; Nan, J.; Fan, L.; Sun, W.; Wang, Y.; Luan, X. Highly Stereoselective Synthesis of Imine-Containing Dibenzo [b, d] azepines by a Palladium(II)-Catalyzed [5+ 2] Oxidative Annulation of $o$-Arylanilines with Alkynes. Angew. Chem. Int. Ed. 2015, 54, 15385-15389.

12. Kayser-Bricker, K. J.; Glenn, M. P.; Lee, S. H.; Sebti, S. M.; Cheng, J. Q.; Hamilton, A. D. Non-peptidic substrate-mimetic inhibitors of Akt as potential anti-cancer agents. Bioorg. Med. Chem. 2009, 17, 1764-1771.

13. Jiang, J.; Zhang, W.-M.; Dai, J.-J.; Xu, J.; Xu, H.-J. Visible-Light-Promoted C-H Arylation by Merging Palladium Catalysis with Organic Photoredox Catalysis. J. Org. Chem. 2017, 82, 3622-3630.

14. Guerra, W. D.; Rossi, R. A.; Pierini, A. B.; Barolo, S. M. "Transition-Metal-Free" Synthesis of Carbazoles by Photostimulated Reactions of 2'-Halo-[1,1'-biphenyl]-2-amines. J. Org. Chem. 2015, 80, 928-941. 
15. Simó Padial, J.; Poater, J.; Nguyen, D. T.; Tinnemans, P.; Bickelhaupt, F. M.; Mecinović, J. Stabilization of 2,6-Diarylanilinum Cation by Through-Space Cation- $\pi$ Interactions. J. Org. Chem. 2017, 82, 9418-9424.

16. Jasch, H.; Scheumann, J.; Heinrich, M. R. Regioselective Radical Arylation of Anilines with Arylhydrazines. J. Org. Chem. 2012, 77, 10699-10706.

17. Yang, L.; Zhang, Y.; Zou, X.; Lu, H.; Li, G. Visible-light-promoted Intramolecular C-H Amination in Aqueous Solution: Synthesis of Carbazoles. Green Chem. 2018, 20, 1362-1366.

18. Smith, G.; Martin, N.; Menear, K.; Hummersone, M.; Cockcroft, X.-L.; Frigerio, M.; Griffin, R.; Golding, B.; Hardcastle, I.; Newell, D. R.; Calvert, H. A.; Curtin, N. J.; Desage-El Murr, M. Preparation of Chromen-4-ones and Their Analogs as DNA-PK Inhibitors. US Patents, 2006109084, October 19, 2006: 2006.

19. Albaugh, P. A.; Dominguez-Manzanares, E.; Hong, J. E.; Hornback, W. J.; Jiang, D.; Ornstein, P. L.; Thompson, M. L.; Tromiczak, E. G.; Wu, Z.; Zarrinmayeh, H. Pyrrole and Pyrazole Derivatives as Potentiators of Glutamate Receptors. US Patent, 7,625,932, December 1, 2009: 2009.

20. Trost, B. M.; Pissot-Soldermann, C.; Chen, I. A Short and Concise Asymmetric Synthesis of Hamigeran B. Chem. Eur. J. 2005, 11, 951-959.

21. Xi, J.; Dong, Q.-L.; Liu, G.-S.; Wang, S.; Chen, L.; Yao, Z.-J. Efficient Synthesis of Phenanthridines Using Hendrickson Reagent Initiated Cascade Reaction under Mild Conditions. Synlett 2010, 2010, 1674-1678.

22. Kim, J.; Moon, Y.; Lee, S.; Hong, S. A Pd-Catalyzed One-pot Dehydrogenative Aromatization and Ortho-functionalization Sequence of $\mathrm{N}$-Acetyl Enamides. Chem. Commun. 2014, 50, 3227-3230.

23. Annamalai, P.; Hsu, K.-C.; Raju, S.; Hsiao, H.-C.; Chou, C.-W.; Lin, G.-Y.; Hsieh, C.-M.; Chen, P.-L.; Liu, Y.-H.; Chuang, S.-C. Palladium(II)-Catalyzed Mono-and Bis-alkenylation of $\mathrm{N}$-Acetyl-2-aminobiaryls through Regioselective $\mathrm{C}-\mathrm{H}$ Bond Activation. J. Org. Chem. 2018, 83, 3840-3856.

24. Samanta, R.; Kulikov, K.; Strohmann, C.; Antonchick, A. P. Metal-Free Electrocyclization at Ambient Temperature: Synthesis of 1-Arylcarbazoles. Synthesis 2012, 44, 2325-2332.

25. Chinnagolla, R. K.; Jeganmohan, M. Ruthenium-catalyzed Ortho-arylation of Acetanilides with Aromatic Boronic Acids: An Easy Route to Prepare Phenanthridines and Carbazoles. Chem. Commun. 2014, 50, 2442-2444.

26. Hyodo, K.; Hasegawa, G.; Oishi, N.; Kuroda, K.; Uchida, K. Direct and Catalytic Amide Synthesis from Ketones via Transoximation and Beckmann Rearrangement under Mild Conditions. J. Org. Chem. 2018, 83, 13080-13087.

27. Antonchick, A. P.; Samanta, R.; Kulikov, K.; Lategahn, J. Organocatalytic, Oxidative, Intramolecular $\mathrm{C}-\mathrm{H}$ Bond Amination and Metal-Free Cross-Amination of Unactivated Arenes at Ambient Temperature. Angew. Chem. Int. Ed. 2011, 50, 8605-8608.

28. Arumugam, V.; Kaminsky, W.; Nallasamy, D. Pd(II) Pincer Type Complex Catalyzed Tandem C-H and N-H Activation of Acetanilide in Aqueous Media: A Concise Access to Functionalized Carbazoles in A Single Step. Green Chem. 2016, 18, 3295-3301.

29. Natarajan, P.; Chuskit, D. Transition-metal-free and Organic Solvent-free Conversion of Nsubstituted 2-Aminobiaryls into Corresponding Carbazoles via Intramolecular Oxidative Radical Cyclization Induced by Peroxodisulfate. Green Chem. 2017, 19, 5854-5861. 
30. Wang, S.; Mao, H.; Ni, Z.; Pan, Y. Pd(II)-catalyzed Intramolecular C-H Activation/C-C Cross Coupling for the Synthesis of Carbazoles from Diaryl Acetamides. Tetrahedron Lett. 2012, 53, 505-508.

31. Cho, S. H.; Yoon, J.; Chang, S. Intramolecular Oxidative $\mathrm{C}-\mathrm{N}$ Bond Formation for the Synthesis of Carbazoles: Comparison of Reactivity Between the Copper-catalyzed and Metalfree Conditions. J. Am. Chem. Soc. 2011, 133, 5996-6005.

32. Tsang, W. C. P.; Zheng, N.; Buchwald, S. L. Combined C-H Functionalization/C-N Bond Formation Route to Carbazoles. J. Am. Chem. Soc. 2005, 127, 14560-14561.

33. Lucchetti, N.; Scalone, M.; Fantasia, S.; Muniz, K. An Improved Catalyst for Iodine(I/III)Catalysed Intermolecular C-H Amination. Adv. Synth. Catal. 2016, 358, 2093-2099.

34. Samanta, R.; Bauer, J. O.; Strohmann, C.; Antonchick, A. P. Organocatalytic, Oxidative, Intermolecular Amination and Hydrazination of Simple Arenes at Ambient Temperature. Org. Lett. 2012, 14, 5518-5521.

35. Hyodo, K.; Hasegawa, G.; Oishi, N.; Kuroda, K.; Uchida, K. Direct and Catalytic Amide Synthesis from Ketones via Transoximation and Beckmann Rearrangement under Mild Conditions. J. Org. Chem. 2018, 83, 13080-13087.

36. Zhou, S.; Wang, J.; Zhang, F.; Song, C.; Zhu, J. A Versatile, Traceless C-H Activation-Based Approach for the Synthesis of Heterocycles. Org. Lett. 2016, 18, 2427-2430.

37. Collins, K. D.; Glorius, F. A Robustness Screen for the Rapid Assessment of Chemical Reactions. Nat. Chem. 2013, 5, 597-601.

38. Hyun, S.-M.; Yuan, M.; Maity, A.; Gutierrez, O.; Powers, D. C. The Role of Iodanyl Radicals as Critical Chain Carriers in Aerobic Hypervalent Iodine Chemistry. Chem 2019, 5, 23882404.

39. Drago, R. S. Physical Methods for Chemists. 2 ed.; Surfside Scientific Publishers: Gainesville, FL, 1992. 